Aus dem Krankenhause St. Göran zu Stockholm.

\title{
Klinische Beobachtungen über die Prognose der kongenitalen Syphilis.
}

\author{
Von
}

Dr. med. Karl Mareus,

z. Z. Vorstand der 1I. Abteilung.

Die Frage der Prognose der syphilitischen Krankheit ist eine der schwierigsten, ja vielleicht die allerschwierigste der ganzen Lueslehre. Wenn wir uns auf dem Gebiete der Ätiologie, der Anatomie, der Symptomatologie, der Diagnose und der Therapie bewegen, sind wir ja auf festerem Boden u. zw. großenteils dank den bedeutungsvollen Entdeckungen des letzten Jahrzehntes. Wenn wir aber ein Urteil über die Zukunft eines Luetikers mit frischer Krankheit aussprechen sollen, wenn wir eine Antwort auf seine ängstlichen Fragen zu geben genötigt sind, dann sind wir in einer viel heikleren Lage.

Der Verlauf der Syphilis muß, wie alle anderen Erscheinungen der Natur, gewissen bestimmten Gesetzen folgen, und diese Gesetzmäßigkeit ist uns ja im großen und ganzen wohlbekannt. Wir kennen die Erscheinungen, die in den ersten Jahren der Lues regelmäßig auftreten; wir kennen auch die schweren Schädigungen lebenswichtiger Organe - z. B. Zirkulationsorgane, Rückenmark, Gehirn - welche gewöhnlich einer späteren Periode der syphilitischen Krankheit angehören und welche den Tod des Syphilitikers verursachen können. Aber wir wissen auch, daß wir durch eine geeignete Behandlung mit spezifischen Mitteln diesen Verlauf wesentlich beeinflussen, Rezidive verhindern, ja die Krankheit heilen können. 
Sich selbst überlassen kann eine Syphilisinfektion ausnahmsweise heilen, wie die Erfahrung gelehrt hat; aber als Regel schreitet die Krankheit fort, unter leichteren oder schwereren Erscheinungen, diesmal das eine, dasmal das andere Organ angreifend. Glücklicherweise besitzen wir so wirksame spezifische Heilmittel gegen die Syphilis wie gegen keine andere so ernste Krankheit. Die Aussichten eines Syphilitikers, den schweren Folgen seiner Krankheit zu entgehen, d. h. die Prognose, sind also davon abhängig, wie es unserer Therapie gelingt, die Spirochaeten, ehe sie diese gefährlichen Schädlichkeiten bewirkt haben, zu vernichten. Nun wissen wir, daß der Grund zu diesen ernsten Veränderungen wichtiger Organe schon in einem sehr frühen Stadium der Krankheit gelegt werden kann. In einem solchen Falle können wir eine Heilung der Erscheinungen durch später einsetzende Behandlung nicht erwarten. In anderen Fällen wurde eine frühzeitige, kräftige und zielbewußte Therapie nicht durchgeführt, sei es zufolge mangelnder Kenntnisse des Arztes, sei es Schuld des Patienten selbst. Aus diesen beiden Kategorien stammt sicherlich die Mehrzahl der Tabiker und Paralytiker, wie aus den veröffentlichten großen Statistiken hervorgeht. Selbstverständlich sind es auch die Neurologen, welche, da sie lauter solche Fälle sehen, betreffs der Prognose der Syphilis am meisten pessimistisch sind.

Im scharfen Gegensatz hierzu stehen ja die Ansichten der Mehrzahl der Syphilidologen, welche die geheilten Fälle beobachten können. Alfred Fournier z. B., der sicherlich zu denjenigen, die die größte Erfahrung über die Syphilis haben, zu rechnen ist, beantwortet die Frage: „En guérit-on?" entschieden mit "Ja“, unter gewissen Bedingungen und nicht nur für den Augenblick, sondern aucb für die Zukunft; seine Bedingungen sind: Behandlung und Hygiene.

In Konsequenz hiermit hat er anch als erster die kräftige, chronisch-intermittente, präventive $\mathrm{Hg}$-Behandlung eingeführt, die allmählich, von hervorragenden Syphilidologen, wie Neisser, Welander u. a., kräftig empfohlen, allgemeine Ausbreitung gefunden hat. $\mathrm{Ob}$ die Salvarsanbehandlung die günstigen Resultate der $\mathrm{Hg}$-Therapie noch verbessern kann, ist 
Klin. Beobachtungen über die Prognose der kong. Syphilis.

wohl zweifelhaft und jedenfalls nicht in Jahrzehnten zu beurteilen. Für den modernen Syphilidologen gilt es also: jede frische Syphilis so frühzeitig und so kräftig wie möglich während der nächsten Jahre zu behandeln; selbstverständlich muß der Patient auch nach der Beendigung der Kuren unter klinischer und serologischer Kontrolle stehen.

Wie die erworbene Syphilis verhält sich ja auch die angeborene. Die Frucht wird in Utero von, der mütterlichen Krankheit entstammenden, Spirochaeten angesteckt, kann also mit einem frisch angesteckten Syphilitiker gleichgestellt werden. Dieser wird sofort behandelt; jene sollte auch logischerweise die spezifischen Mittel bekommen. Dies läßt sich ja auch durch die Behandlung der graviden Mutter bewerkstelligen; wissen wir ja durch vielfache Untersuchungen, daß sowobl $\mathrm{Hg}$ wie $\mathrm{Kj}$ und As vom mütterlichen Kreislaufe in den fötalen iibergeht. Die segensreichen Resultate dieser „intrauterinen “ Behandlungsmethode werden auch von Fournier, Welander u. a. in mehreren Publikationen nachdrücklich hervorgehoben. Und Welander geht noch einen Schritt weiter, indem er die chronisch-intermittente, präventive $\mathrm{Hg}$-Behandlung auch den Kindern zugängig gemacht hat, durch die Einrichtung des ersten Asyles für kongenitalluetische Kinder, das 1900 in Stockholm eröffnete "Lilla hemmet".

Jetzt ergibt sich von selbst die Frage: Wie haben sich diese therapeutischen Anschauungen bewährt?

Diese Kinder, welche während der $3-4$ ersten Lebensjahre spezifisch behandelt werden, außerordentlich selten ansteckende syphilitische Erscheinungen bekommen, sich körperlich und geistig normal entwickeln, bleiben sie auch gesund? Bekommen sie nicht in der Zukunft die ernsten Schädigungen wichtiger Organe, die wir in dem Begriffe Lues hereditaria tarda zu vereinigen gewöhnt sind? Vielleicht werden sie doch einmal der Gesellschaft zur Last fallen und damit die erheblichen Kosten für solche Asyle, die aufopfernde Pflege, ja den ganzen großen Gedanken zunich te machen?

Endgültig diese Fragen zu beantworten ist ja noch die Zeit zu kurz - die erste Anstalt ist ja nur 12 Jahre alt. Aber 
etwas können wir schon sagen, ja vieles und glïcklicherweise -Günstiges.

Es sei also das Ziel dieser Arbeit, mit Hilfe des mir zugängigen Materials nachzusehen, wie die Resultate der intrauterinen und fortgesetzten Behandlung der Kinder sich gestaltet haben, mit anderen Worten, ob wir auf Grund der vorliegenden Erfahrungen die Prognose, die ja im allgemeinen als sehr ungünstig angesehen wird, für diese kleinen Patienten besser stellen können, als für jene, die nicht nach den erwähnten $\mathrm{Me}-$ thoden behandelt waren.

Die traurigen Schicksale der ungenügend oder gar nicht behandelten Kinder mit angeborener Lues kennen wir ja leider gut. Edmond F o urnier, Hochsinger und neuerdings Sprintz haben unter anderen in den letzten Jahren von verschiedenen Gesichtspunkten aus dies Thema behandelt. Da es nicht im Plane dieses Aufsatzes liegt, eine ausführliche Historik zu geben, gestatte ich mir auf eine solche hjer zu verzichten und betreffs des Geschichtlichen auf die genannten Autoren hinzuweisen.

Nur einige wichtige Tatsachen erachte ich als ratsam zu erwähnen, um den folgenden Auseinandersetzungen den nötigen Hintergrund zu geben.

Zuerst will ich da in Erinnerung rufen, wie die alte Lehre von der paternen Syphilis, das Coll e s-B e a um ès sche und Profetasche Gesetz, durch die Arbeiten von Oedmanns on und vor allem durch Matzenauer in seinen Grundlagen kräftig erschüttert, in den allerletzten Jahren durch die Serodiagnostik und den Nachweis der Spirochaeten im Plazentargewebe (Ba is ch u. a.) definitiv gestürzt wurde. Für uns gibt es nur eine Form der angeborenen Lues, die materne. Die Frucht wird in Utero oder beim Partus (Rietschel) von der Mutter angesteckt. Eine Infektionskrankheit kann man ja nicht vererben; die Benennung "Erbsyphilis“, „hereditäre Syphilis" ist also nicht zutreffend. Daher muß man diese Bezeichnungen vermeiden und lediglich von einer angeborenen, kongenitalen Lues reden (Krefting).

Die unmittelbare Folge einer solchen Anschauung ist, daß ein großer Teil der Umstände, welche bisher in Betracht 
gezogen wurden, wegfallen, d. i. alles was den Vater betrifft. Das Alleinbestimmende für einen solchen Versuch muB die mütterliche Krankheit sein, welche aus den Gesichtspunkten des Alters, der Schwere und der durchgemachten Behandlung zu beurteilen ist.

Was das Alter der maternellen Krankheit betrifft, so scheinen die Ansichten hierüber ziemlich einig zu sein. Alle Lehrbücher, die ich kenne, haben dieselbe Darstellung : je älter die Syphilis, desto weniger gefährlich für die Frucht. Sicherlich trifft dies sehr oft zu, aber nicht ausnahmslos, wie ich aus der folgenden Darstellung meiner Beobachtungen feststellen zu können hoffe. Und dies kann nicht verwundern. Ans den neuen wissenschaftlichen Tatsachen geht ja hervor, daß keine kongenitale Syphilis zustande kommen kann, wenn Spirochaeten nicht von der Mutter auf das Kind übergehen. Und diese Mikrorganismen kommen in jedem beliebigen Stadium der Syphilis vor; sie sind in Gummen der verschiedensten Lokalisation nachgewiesen, z. B. bei der Aortitis luetica. Zwar sind sie, wie bekannt, bei älterer Syphilis spärlicher vorhanden, in vereinzelten Herden lokalisiert als bei frischer Krankheit, wo der ganze Organismus vom Virus durchgeseucht sein kann. Die Aussichten für ein Kind, e in er intrauterinen Infektion zu entgehen, sind demnach theoretisch größer, je älter die mütterliche Syphilis ist.

Gebärt nun eine Frau mit alter Syphilis ein Kind mit luetischen Symptomen, hat man jetzt eine Richtschnur für die Annahme, daß die Aussichten dieses Falles günstiger sind als wenn die mütterliche Krankheit frisch wäre? Das hängt von der Virulenz der Spirochaeten bei den verschiedenen Stadien der Lues ab. Soviel ist uns bekannt, daß Gummen anstecken können und daß nässende Papeln mit sehr lebenskräftigen Spirochaeten auch Jahrzehnte nach der Infektion entstehen können. Nichts steht daher im Wege, daß auch bei alter Syphilis der Mutter ein kräftiges Virus die Frucht anstecken kann - solche Fälle werde ich unten anführen.

Die Schwere der mütterlichen Krankheit zu beurteilen; ist bei einigen Fällen sehr leicht; wenn ausgebreitete, tiefgreifende oder hartnäckige Erscheinungen vorhanden sind, wenn innere Organe angegriffen sind, wenn im gegebenen Falle die 
spezifische Therapie nur wenig oder gar nicht einwirkt - dann liegt ein schwerer Fall vor. Falls dagegen keine äußerlichen oder inneren Erscheinungen zu beobachten sind, stellt sich die Frage schwieriger. Hier könnte die Serodiagnostik uns große Dienste leisten, aber nur in einzelnen Fällen - hartnäckige positive Reaktion, die kräftiger, antiluetischer Behandlung nicht weicht oder konstante negative Reaktion, in beiden Fällen durch Jahre fortgesetzte Beobachtung - haben wir darin eine Stütze zur Beurteilung der Schwere der mütterlichen Syphilis. Warum der eine Fall schwerer verläuft als der andere, ist bisher nicht erklärt; die parasitologische und die serologische Forschung wird uns wohl in der Zukunft die Antwort geben. Vorläufig interessiert uns hier die Frage, ob die Beschaffenheit der mütterlichen Syphilis einen EinfluB auf die des Kindes hat. Bei unseren mangelhaften Kenntnissen der näheren Umstände, welche den Ausgang des Kampfes zwischen den Spirochaeten und dem menschlichen Organismus bestimmen, ist ein theoretisches Räsonnieren fast wertlos. Wenn wir dagegen nachschauen, wie es sich in der Wirklichkeit verhält, werden wir keine RegelmäBigkeit in dieser Hinsicht finden. Eine symptomenfreie Mutter, sogar mit negativer W. R., kann ein schwerkrankes Kind gebären und umgekehrt. Beispiele in beiden Richtungen werden wir unten finden.

Auf festerem Boden befinden wir uns aber, wenn wir den dritten Faktor, der auf die Folgen einer syphilitischen Schwangerschaft einwirken kann, die vor und während der Gravidität gegebene antiluetische Behandlung in Betracht ziehen. Daß eine zielbewußte, kräftige und langwierige $\mathrm{Hg}$-Behandlung einen günstigen Einfluß anf die Syphilis ausübt, ja, in vielen Fällen eine wirkliche Heilung herbeiführen kann, ist nunmehr als ein Faktum anzusehen; ob das Salvarsan bessere Dauerresultate leisten wird, halte ich für mindestens zweifelhaft, wenigstens nur nach Jahrzehnten zu entscheiden. Als ein Kriterium um den Erfolg der Hg-Behandlung zu beurteilen, sind Rezidive und Serorealtion zu betrachten. Der große Unterschied in diesen Beziehungen zwischen kräftig und schlecht behandelten Individuen ist ja eine anerkannte Tatsache. Tabellen und Diagramme, auf ein großes Material gestützt, welche diese 
Klin. Beobachtungen über die Prognose der kong. Syphilis. 103

Verhältnisse scharf beleuchten, habe ich in diesem Archiv (Band CVII, Heft 1-3) veröffentlicht.

Hier wird uns nur interessieren, ob die spezifische Behandlung der Mutter einen Einfluß auf die Lebensaussichten der kranken Kinder ausüben kann. Die meisten Autoren, welche in dieser Beziehung größere Erfahrung besitzen, ziehen einen solchen günstigen EinfluB nicht im Geringsten in Zweifel. (Fournier, Welander, Bering u. a.) Trotzdem gibt es andere, welche in dieser Frage eine ganz andere Erfahrung haben; die Erklärung siehe oben; Ausnahmsfälle gibt es überall. Einige solche traurige Beobachtungen teile ich unten mit; die Mehrzahl meiner Fälle dagegen sprechen glücklicherweise eine ganz andere Sprache.

Um eine möglichst zuverlässige Antwort auf das aufgestellte Problem zu erhalten, wäre es ja absolut ungenügend, die Beobachtung auf den relativ kurzen Aufenthalt im Krankenhause zu beschränken, vielmehr wäre es nötig, die Observation so lange Zeit wie möglich auszudehnen. Eine Gelegenheit hierzu bietet das "Lilla hemmet"; die meisten der kleinen Patienten sind als Neugeborene im Krankenhause St. Göran gepflegt worden; nach dem Entlassen nach dem gewöhnlichen dreijährigen Aufenthalte im Asyl haben sich mehrere von Zeit zu Zeit gezeigt, um untersucht zu werden. Für die freundliche Überlassung dieses Materials spreche ich Herrn Professor Edward Welander meinen tiefsten Dank aus.

Ich habe meine Fälle von Säuglingssyphilis in drei große Gruppen verteilt, nach dem Alter der mütterlichen Syphilis.

I. die Iufelktion der Mutter erfolgte:

a) nach der Konzeption,

b) gleichzeitig mit derselben und

c) kurz (höchstens einige Monate) vor derselben.

II. Die Infektion der Mutter erfolgte:
a) 1-2 Jahre,
b) $2-3$
c) $3-4$, 
d) höchstens 4 Jahre und

e) mehr als 4 Jahre vor der Geburt des Kindes

III. Die Infektion der Mutter zeitlich unsicher.

Diese Einteilung habe ich aus folgenden Gründen gemacht. Wenn der gravide mütterliche Organismus von Spirochaeten durchseucht ist, müssen die Aussichten der Frucht einer Infektion $\mathrm{zu}$ entgehen, viel geringer sein, als wenn sich dieselben nur in vereinzelten Herden vorfinden. - Wenn die Ansteckung der Mutter kurz vor, gleichzeitig mit oder nach der Schwängerung (die allerletzte Zeit der Gravidität ausgenommen) eintraf, folgt fast ausnahmslos eine solche Durchseuchung während des intrauterinen Lebens des Kindes Gruppe I. Die zweite Gruppe umfaßt ältere Fälle; die Aussichten, daß während der Schwangerschaft eine solche gefährliche Überschwemmung von Spirochaeten eintreten kann, sind bei diesen Fällen viel geringer; selbstrerständlich $\mathrm{kann}$ so was vorkommen bei Allgemeinrezidiven während der ersten Krankheitsjahre, aber später nicht. In einer dritten Gruppe (III) habe ich diejenigen Fälle zusammengestellt, bei denen die Infektionszeit der Mütter sich nicht feststellen ließ.

Das Material umfaßt alle Patienten des "Lilla hemmet" und die Mehrzahl der kongenitalluetischen Kinder, die im Krankenhause St. Göran seit 1908 (in diesem Jabr ist die Serodiagnostik hier zuerst angewandt worden) aufgenommen sind; nur die spärlichen Fälle, welche zufolge unvollständiger Krankengeschichten mir unbrauchbar schienen, sind ausgeschlossen. Die Krankengeschichten sind nicht in Extenso angeführt, sondern nur die Einzelheiten, welche zur Beurteilung des Falles ron Bedeutung sind, werden summarisch mitgeteilt.

Ehe ich zur Besprechung meines eigentlichen Themas übergehe, werde ich 13 Fälle von sog. Lues hereditaria tarda, die während der letzten 4 Jahre im Krankenhause beobachtet wurden, in aller Kürze erwähnen. Sie zeigen alle die diesem Krankheitsbegriffe zugehörigen Veränderungen in wechselnder Ausbreitung; was uns hier interessiert, ist das Alter, bei welchem die jetzt erwähnten Symptome zuerst auftraten. Bering 
hat auf Grund seiner Fälle diesen Zeitpunkt durchschnittlich auf das achte Lebensjahr verlegt, F ournier gibt das zwölfte $\mathrm{Jahr}$ an und andere Autoren im allgemeinen das achte bis zwölfte Jahr. Für mein Material (Fälle 1-12) wird der Zeitpunkt für das erste Auftreten der betreffenden Erscheinungen auf das zehnte Jahr ungefähr berechnet; wenn nähere Angaben zur Verfügung wären, glaube ich doch, daß ein noch jüngeres Lebensjahr das Resultat gewesen wäre. Da die ältesten Kinder des "Lilla hemmet" sich jetzt im zwölften bis dreizehnten Lebensjahre befinden, so wäre ja zu erwarten, daß bei einigen von ihnen solche Erscheinugen einer sog. tardiven Syphilis aufgetreten wären, wenn die Behandlung das Auftreten solcher nicht hätte verhindern können.

Der Fall 13 bietet ein ganz besonderes Interesse, weil es sich hier möglicherweise um eine Syphilis der dritten Generation handelt. Um einen solchen Vorgang sicher feststellen zu können, muß man ja die kongenitale Lues des zweiten und dritten Gliedes der Reihe einwandsfrei festgestellt und eine neue Infektion des zweiten Gliedes ausgeschlossen haben. Die ziemlich zahlreich veröffentlichten Fälle erfüllen auch die erste dieser Bedingungen; Großmutter, Mutter und Kind sind z. B. von demselben Syphilidologen beobachtet worden. Die zweite Bedingung dagegen ist schwieriger zu erfüllen; wie die erworbene kann ja die kongenitale Lues spontan oder unter spezifischer Behandlung ausheilen (Reinfektionen, Tierversuche von $\mathrm{Neisser} u$. a.). In solchen Fällen stößt ja eine neue Ansteckung nicht auf das Hindernis, welches ein syphilitischer Organismus derselben bietet; Immunität im strengen bakteriologischen Sinne gibt es ja bei der Syphilis nicht ( $\mathrm{N}$ e is ser).

Laut Krankengeschichte wurde die Großmutter im Sommer 1887 infiziert, bekam nur $4 \mathrm{Hg}$-Injektionen und gebar im Frühjahr 1888 die Patientin; ob diese etwaige syphilitische Symptome damals hatte, ist nicht festzustellen. Die feinen Narben in den Mundwinkeln, welche ich bei der Untersuchung 24 Jahre später wahrnahm, deuten doch auf vorhergegangene Rhagaden. Die radiären Narben werden ja als das sicherste von allen den 
sog. Stigmata angesehen (Hochsinger, Bering u. a.). Auch ein anderer Umstand stärkt die Annahme einer kongenitalen Lues in diesem Falle: Eine im folgenden Jahre geborene Schwester der Patientin starb im zweiten Lebensmonate, nach den Angaben an Bronchitis. Legen wir jetzt die oben erwähnten drei Tatsachen zusammen, die frische Ansteckung der Großmutter $3 / 4$ Jahre vor der Geburt der Patientin, die für Kongenitallues charakteristischen Narben an den Lippensäumen und der frühzeitige Tod der Schwester, so wird es ja nicht nur böchst wahrscheinlich, sondern fast sicher, daß die Patientin mit angeborener Lues behaftet war. Ihr Kind (Fall 99), das ich selbst beobachtet habe, hatte solche Symptome, daB an der kongenitalen Syphilis desselben kein Zweifel sein kann. Somit ist die erste Bedingung, sichere angeborene Lues der zweiten und dritten Generation, in diesem Falle so nahe wie möglich erfüllt.

Die Hg-Behandlung, welche die Großmutter während der Schwangerschaft durchmachte, hat zwar die Frucht nicht vor einèr Infektion retten können, aber doch vor schwereren syphilitischen Veränderungen; während der Kinderjahre soll sie nichts spezifisches aufgewiesen haben. $\mathrm{Daß}$ sie jedoch nicht von ihrer Krankheit geheilt war, geht unzweideutig aus der Geschichte ihrer Schwangerschaften hervor. Zuerst in ihrem achtzehnten Lebensjahre totgeborene luetische Frucht (nach der in der Städtischen Gebäranstalt zu Stockholm geführten Krankengeschichte); folgendes Jahr wieder eine luetische Fehlgeburt; nächstes Jahr wieder gravid, bekam sie eine $\mathrm{Hg}$-Kur, dank deren wohltuender Wirkung sie ein ausgetragenes symptomfreies Kind gebar. Dieses Kind war vier Jahre alt frei von anderen Erscheinungen als einer Asymmetrie des Kopfes, ein Symptom, welches jedoch von manchen Autoren (z. B. E. Fournier) zu den Stigmata der kongenitalen Lues gerechnet wird, und gab negative W. R. Die nächste Geburt, die des jetzt in Frage kommenden Kindes, wurde wieder luetisch; Patientin hatte seit vier Jahren kein Hg bekommen. Bei der Untersuchung hatte sie positive W. R. - Liese jetzt geschilderten Fakta beweisen wohl, daß sie während der letzten sechs Jahre luetisch geweser ist. Gegen die Annahme einer 
Klin. Beobachtungen über die Prognose der kong. Syphilis. 107

neuen Ansteckung sprechen: erstens, daß sie bei der Untersuchung kein einziges Zeichen einer frischen Infektion hatte und zweitens ihre bestimmte Versicherung, mit keinem anderen Manne als mit dem Ehemanne verkehrt zu haben; dieser verneinte syphilitische Infektion, hatte keine Erscheinungen von Lues und gab negative W. $R$.

Die zweite Bedingung, eine neue Infektion des zweiten Gliedes ausschließen zu können, scheint mir durch diese jetzt angeführten Verhältnisse so vollständig wie möglich erfüllt zu sein. Der Fall ist demnach, meiner Ansicht nach, als ein Beispiel der kongenitalen Lues der dritten Generation aufzuf assen.

Ich gehe jetzt zu den näheren Untersuchungen meines Materials von Säuglingssyphilis über, und werde zuerst nach dem aufgestellten Schema die einzelnen Gruppen und Abteilungen jede für sich besprechen, um nachher mit Hilfe aufgestellter Tabellen das Ganze zu verwerten. Dieser Teil meiner Arbeit umfaßt 114 Fälle.

Weiterhin werden die Schicksale der Patienten des „Lilla hemmet" auf Grund der tabellarischen Übersicht in Betracht kommen und näher beurteilt. Das kleine Asyl hat bis jetzt 70 Kinder beherbergt; ein Mädchen war jedoch in den ersten Lebensmonaten mit Syphilis angesteckt worden und wird demnach, als dem Material dieser Arbeit nicht zugehörig, gar nicht in Betracht gezogen. Die Mehrzahl der Kinder sind als Neugeborene im Krankenhause St. Göran aufgenommen und kommen deswegen auch unter den Fällen der ersten Abteilung vor, was in jeder Krankengeschichte der Kasuistik angegeben ist.

Wie aus der Kasuistik hervorgeht, verfüge ich über 13 Fälle von postkonzeptioneller Infektion. Daß es sich um eine wirkliche Infectio post conceptionem handelt, geht nicht nur aus den anamnestischen Angaben hervor, sondern vielmehr daraus, daß alle 13 Mütter bei der Geburt des Kindes 
solche Symptome (Sklerose oder Sklerosenreste, noch kein Leukoderma), welche jünger als die Schwängerung sein müßten, aufgewiesen haben. Die jüngste Ansteckung war 2 Monate, die älteste 8 Monate vor dem Partus.

6 Patientinnen (Fälle 14-19) hatten keine Behandlung erhalten: von deren Kindern hatten 5 bei der Geburt Erscheinungen aufzuweisen. Unter "Erscheinungen" oder "Symptome" verstehe ich hier sowie unten nicht nur klinische, äußere oder innere syphilitische Veränderungen, sondern auch positive Wassermannsche Reaktion (W. R.), gleichzeitig mit oder kurz nach der Geburt. - Nur ein Kind (Fall 19) zeigte sich völlig symptomfrei und hatte bei drei aufeinander folgenden Untersuchungen vollständig negative W. R., starb aber 4 Monate alt an Pneumonie.

2 Mütter waren mit Salvarsan behandelt; die eine (Fall 20) batte eine Injektion, die zweite (Fall 21) 3 Injektionen à 0.4 bekommen: beide Kinder zeigten unzweideutige luetische Symptome, das zweite zwar negative Reaktion, ging aber 14 Tage alt durch Verblutung rom Nabel zu grunde.

5 Mütter waren mit $\mathrm{Hg}$ behandelt; zwei Kinder hatten syphilitische Erscheinungen; die Behandlung war in dem einen Falle (Nr. 22) Hg-Säckchen à $7 g$ ung. Hydrargyri 19 Tage, also ungenügend, im zweiten Falle (Nr. 23) waren 10 Injektionen von Merkuriolöl à $0.067 \mathrm{~g} \mathrm{Hg}$ gegeben. - Der Kürze wegen werden $\mathrm{im}$ folgenden $\mathrm{Hg}$-Säckchen $=\mathrm{Hg} \mathrm{S}$., Merkuriolölinjektionen $=\mathrm{Mk}$ und Einspritzungen rom Salicylas hydrargyricus = Sal. Hg gezeichnet.

Die 3 übrigen Mütter, welche symptomfreie Kinder gebaren, hatten $\mathrm{Hg}$ à $6 g 40$ Tage, Fall 24, do. + 4 Sal. Hg, Fall 25, und do. +6 Sal. Hg, Fall 26, erhalten.

Die Aussichten für die Frucht, einer Ansteckung zu entgehen, scheinen also bei postkonzeptioneller Infektion der Mutter, auch wenn diese erst im 7. Graviditätsmonat erfolgte, sehr gering zu sein. Auch wenn eine spezifische Behandlung durchgeführt wird, sind die Resultate für das Kind nicht so günstig wie bei älterer Krankheit der Mütter, wie unten gezeigt werden soll. Und das ist ja nicht zu verwundern. 
Erstens erfolgt bei der postkonzeptionellen Infektion die erste Überschwemmung des mütterlichen Organismus mit Spirochaeten fast sicher vor dem Partus, und daraus folgt selbstverständlich, daß die Wahrscheinlichkeit für die Frucht einer Ansteckung zu entgehen, sehr gering sein muß. Zweitens kann die spezifische Behandlung zufolge der auf einige Monate beschränkten Zeit keine Dauerwirkung, eventuell eine vollständige Abtötung der Spirochaeten, wie wir mit der durch Jahre fortgesetzten chronisch-intermittenten, präventiven $\mathrm{Hg}$-Behandlung beabsichtigen, mitführen. Daß die Therapie auch bei den betreffenden Umständen doch für das Kind von großem Nutzen sein kann, zeigen doch die drei obenerwähnten Kinder (Fälle 24-26), welche symptomfrei zur Welt kamen.

I b)

Infektion ungefähr gleichzeitig mit der Konzeption.

Erfolgte die Schwängerung der Frau ungefähr gleichzeitig mit der Ansteckung, liegen die Verhältnisse ungefähr wie oben gesagt wurde. Die Infektionsmöglichkeit für die Frucht mub in Anbetracht, daß der allgemeine Ausbruch der mütterlichen Syphilis während der Schwangerschaft erfolgen muß, überaus groß sein. Es geschieht auch unter diesen Umständen, wo die Mehrzahl der Früchte schon in einem frühen Stadium des intrauterinen Lebens durch das syphilitische Virus getötet werden, daß die meisten Aborte zustande kommen. Ich habe während des Jahres 1911166 Sera von Müttern, welche in der geburtshilfl. Klinik zu Stockholm Fehlgeburten oder Fruihgeburten gebaren, nach Wassermann untersucht; 29 von ihnen $(17 \cdot 2 \%)$ gaben positive W. R.; 21 waren im zweiten bis siebenten Graviditätsmonat, die übrigen im achten und neunten. 21 Mütter waren klinisch symptomfrei, acht hatten Erscheinungen; alle diese gehörten der ersten Sekundärperiode. Zwei Früchte hatten syphilitische Ausschläge, acht waren mazeriert.

Wenn die Früchte aber lebensfähig geboren werden und keine Behandlung während der Schwangerschaft dazwischen kommt, wäre es ja zu vermuten, daß kein einziges einer Infektion ent- 
gehen sollte. Von den hierher gehörigen 7 Fällen ist nur ein Kind (Fall Nr. 27) frei von Erscheinungen geboren worden. In diesem Falle erfolgte jedoch die Geburt einen Monat zu früh, das Kind war sehr schwächlich und starb nach einem Jahre an Pneumonie; bei 6 während dieses Jahres vorgenommenen Untersuchungen war die Seroreaktion immer vollständig negativ; das Kind hatte jedoch während derselben Zeit $4 \mathrm{Hg}$-Kuren à 40 Tagen durchgemacht, laut den Grundsätzen, welchen wir nach Welander folgen, jedes auch symptomfreie Kind einer syphilitischen Mutter als syphilitisch zu betrachtea und mithin spezifisch zu behandeln. Wir wissen ja jetzt sicher, daß die Hg-Therapie eine positive Reaktion in den meisten Fällen zum Schwinden bringen kann. (Siehe z. B. die oben zitierte Arbeit rom Verfasser.) Wäre in diesem Falle das spezifische Mittel nicht verabreicht worden, wäre es ja möglich, ja, wahrscheinlich, daB klinische Erscheinungen oder eine positive W. R. aufgetreten wären.

Als eine Regel muß man jedoch glauben, daß kein Kind unter den gegebenen Umständen einer Ansteckung entgehen kann, ja, à priori wäre zu vermuten, daß die kongenitale Syphilis in diesen Fällen besonders schwer verlaufen sollte, wenn man bedenkt, daß die Frucht monatelang der unmittelbaren Einwirkung der mütterlichen Krankheit ausgesetzt ist, ohne jegliche spezifische Behandlung. Die sechs Kinder, welche dieser Gruppe gehören (Fälle Nr. 28--33), haben alle zwar Erscheinungen gehabt, aber nicht besonders schwere; 2 (Fälle 28-29) sind in den ersten Lebensmonaten an akuten Infektionskrankheiten gestorben, 3 sind im "Lilla Hemmet" aufgenommen worden (Fälle 30-32). Die zwei ersten haben sich tadellos entwickelt, der dritte (L. H. 65) ist jetzt nur $3 / 4$ Jahre alt und hat noch positive W. R. Die Anzahl diesbezüglicher Fälle ist ja zu klein, um ein Urteil über die Prognose aussprechen zu können.

Wurden die Mütter aber während der Schwangerschaft spezifisch behandelt, wie gestalteten sich dann die Verhältnisse? 8 von den 13 Kindern wurden frei von allen Erscheinungen geboren; 2, deren Mütter nur schwach behandelt 
waren (Fälle 34-35), hatten zwar bei der Geburt ein nur geringes Gewicht, aber entwickelten sich als Pfleglinge des "Lilla Hemmet" unter den wohltuenden Einwirkungen des Quecksilbers sehr gut. Die 5 Kinder dagegen, welche manifest syphilitisch geboren waren, boten folgende Verhältnisse, welche von Interesse sein können, dar. Im Falle 42 hatte die Mutter nur eine Hg S-kur à 40 Tage durchgemacht; das Kind starb nach 40 Tagen an Pneumonie. Ein Kind (Fall 43) 5 Wochen zu früh geboren, atrophisch (Gewicht $20 j 0 \mathrm{~g}$ ), entwickelte sich während der folgenden Jahre zwar ohne klinische Rezidive zu zeigen, aber hatte noch $3^{1} / 2$ Jahre trotz der konsequent durchgeführten Hg-Behandlung eine hartnäckige positive W. R., welche erst jetzt, wo das Kind 4 Jahre ist, in eine negative übergeführt worden ist; die Mutter hatte nur eine $\mathrm{Hg}$-Säckchen-Kur von 40 Tagen +4 Sal. Hg bekommen. Von einer anderen Mutter (Fall 44) wird nur angegeben, daß3 sie während der Schwangerschaft mit $\mathrm{Hg}$ behandelt wurde; das Kind hatte bei der Geburt Papeln am After und zeigte im 10. Lebensjahre Hutchinsonsche Zähne und positive W. R.; soll während der ersten Lebensmonate nur eine $\mathrm{Hg}$-Kur bekommen haben. Die zwei übrigen Fälle $(45-46)$ betreffen Salvarsanpatienten; die eine Mutter hatte 3, die andere 4 Injektionen à 0.4 erhalten; beide Kinder waren ausgetragen und klinisch symptomfrei, hatten aber positive W. R. Die zweite Mutter bekam kurz nach dem Partus ein schweres Neurorezidiv, doppelseitige Neuritis accustica, welche unter kräftiger $\mathrm{J} \mathrm{K}$ und $\mathrm{Hg}$-Behandlung nach 4 Monaten nicht geheilt ist. Einen besseren Erfolg hatte das Salvarsan im Falle Nr. 45, wo nach 3 Injektionen à 0.4 die Mutter ein ausgetragenes symptomenfreies Kind gebar, welches noch nach 8 Monaten negativ reagiert.

Aus dem obigen geht ja deutlich hervor, welchen wohltuenden Einflub eine spezitische Therapie auf den Zustand des Säuglings ausüben kann; trotzdem die Behandlung in einigen Fällen als wenig kräftig zu bezeichnen war, wurden nicht weniger als 8 von 13 sicher syphilitischen Früchten symptomenfrei geboren. Daß dies nicht gleichbedeutend mit bleibender Gesundheit zu bezeichnen ist, wissen wir ja aus Erfahrung. 
Hier habe ich nur 2 Fälle (Nr. 47, 48). Beide Mütter waren während der Schwangerschaft antiluetisch behandelt, die eine mit Quecksilber, die zweite mit Quecksilber und Salvarsan; beide Kinder batten bei der Geburt Erscheinungen von Syphilis; das der salvarsanbehandelten Mutter wog nur $1900 \mathrm{~g}$ und ging nach einem Monate zugrunde.

Betreffs der Erscheinungen der Mütter bei der Geburt des Kindes wäre es ja zu erwarten, daß sie bei ihrer höchstens 10-11 Monate alten Syphilis auch ausgeprägt primäre oder sekundäre Symptome derselben aufgewiesen haben. Durchmustern wir in der Kasuistik die dieser Gruppe Ia, b, c, angehörigen 35 Fälle, so ersehen wir auch, daß dies der Fall ist.

Die W. R. der Mütter beim Partus bieten bei dem betreffenden Stadium ihrer Syphilis nur wenig Interesse; alle welche keine Behandlung durchgemacht hatten, und welche serologisch untersucht wurden, gaben auch, wie zu erwarten war, positive W. R.

\section{Gruppe II.}

Die zweite Gruppe meiner Fälle umfaßt solche syphilitischen Schwangerschaften, bei welchen die Infektion der Mutter später als ein Jahr vor der Geburt des Kindes erfolgte. Der Zeitpunkt der Ansteckung ist entweder nach den in der Mehrzahl der Fälle genügend kontrollierten anamnestischen Angaben, mangels solcher durch die vorhanden gewesenen klinischen Erscheinungen oder durch beide bestimmt. Der Übersichtlichkeit wegen habe ich die Fälle in kleinere Gruppen verteilt; der Einteilungsgrund sind die klinisch wichtigen Zeitperioden, welche die Lues durch- 
länft, also die ersten 3-4 Jahre jedes für sich und die Fälle, wo die Krankheit älter als 4 Jahre war.

II a) Infektion vor 1 bis 2 Jahren.

2 von den 12 hierher gehörigen Müttern (Fälle 49-50) waren bei der Geburt des Kindes frei von klinischen Erscheinungen. Beide waren vor der Konzeption mit Hektine behandelt worden, die eine hatte während der Schwangerschaft 2mal Salvarsan à 0.4 bekommen und hatte positive W. R. ; das Kind kam atrophisch (Gewicht $2250 \mathrm{~g}$ ) einen Monat zu früh zur Welt, hatte schwach positive W. R. und starb 20 Tage alt an Bronchopneumonie. Die andere Mutter hatte während der Schwangerschaft nur 2 Mk. bekommen: beim Partus positive W. R.; das Kind 1 Monat zu früh geboren, hatte ausgebreitete und schwere syphilitische Symptome nebst positiver W. R. aufzuweisen und starb 11 Tage alt. Die Behandlung war offenbar in beiden Fällen zu schwach, um die Infektion der Früchte zu verhindern oder die positive Seroreaktion zu verändern, obschon kräftig genug, um die Hautund Schleimhauterscheinungen der Mütter zu entfernen.

Bessere Resultate ergeben die 6 Fälle (Nr. 51-56), wo die Mütter während der Schwangerschaft mit $\mathrm{Hg}$ behandelt wurden. Fünf Kinder wurden frei von Erscheinungen geboren, nur eines kam atrophisch zur Welt und starb 4 Monate alt an Gastroenteritis; syphilitische Erscheinungen wurden weder während des Lebens noch bei der Sektion beobachtet. Die Mutter (Fall 56) hatte während der Schwangerschaft 4 Injektionen und Hg-Säckchen bekommen.

Weiter haben wir 4 Fälle, wo die Mütter als Schwangere gar nicht behandelt wurden; 2 von ihnen (Fälle 57, 58) hatten vor der Gravidität $\mathrm{Hg}$-Behandlungen durchgemacht, aber die günstige Wirkung dieser hatte sich nicht über die Zeit der Gravidität erstrecken können, beide Kinder boten ausgebreitete syphilitische Symptome dar. Gar nicht behandelt waren die letzten zwei (Fälle 59, 60). Daß ihre beiden Kinder mit luetischen Erscheinungen geboren werden würden, war ja nicht anders zu erwarten. 
In dieser Gruppe haben wir nur einen Fall (Nr. 61), wo die Mutter niemals behandelt worden war; ausgebreitete syphilitische Erscheinungen beim Kinde ; trotz energischer Hg-Behandlung Rezidiv nach einem Jahre und Tod an Pneumonie nach $1 \frac{1}{2}$ Jahren. Unbehandelt während der Gravidität waren 4 Frauen (Fälle 62-65); 3 Kinder waren syphilitisch. Im Falle Nr. 62 waren $3 \mathrm{Hg}$-Injektionskuren, im Falle $\mathrm{Nr} .635$ solche und im Falle Nr. 642 Salvarsan à 0.45 und 0.60 gegeben worden; das letzte Kind starb 3 Monate alt an Bronchopneumonie; bei der Sektion Hepatitis interstitialis luetica. Die erste Mutter zeigte bei der Geburt des Kindes ein Leukoderma, die andern zwei waren symptomfrei. Das vierte Kind (Fall Nr. 65) wurde ohne Erscheinungen geboren: die Mutter war sehr energisch behandelt worden - $6 \mathrm{Hg}$-Injektionskuren während $1 \frac{1}{12}$ Jahren - und symptomfrei.

10 Mütter wurden sowohl vor wie während der Gravidität spezifisch behandelt. Zwei von ihnen waren beim Partus frei von syphilitischen Erscheinungen; ein Kind (Fall Nr. 66), dessen Mutter während der Schwangerschaft 11 Hg-Injektionen bekommen hatte, hatte nur Coryza und sonst nichts; der weitere Verlauf war auch sehr günstig. Das zweite Kind dagegen (Fall Nr. 67) - während der Gravidität hatte die Mutter etwas mehr als 7 Injektionen bekommen und gab 21/2 Monate nach dem Partus negative W. R., welche jedoch noch einige Monate später, trotz erneuerter $\mathrm{Hg}$-Behandlung absolut positiv wurde, hatte $2 \%$ Monate nach der Geburt ausgebreitete Haut- und Schleimhautsymptome und hartnäckig positive W. R.

Acht Frauen hatten mehr weniger ausgebreitete syphilitische Erscheinungen; von deren Kindern waren vier frei und vier behaftet mit syphilitischen Erscheinungen. Im Falle Nr. 68 waren die Erscheinungen des Säuglings sehr gering - Coryza, negative W. R. - aber schon nach einem Monate wurde die W. R. positiv; weiter siehe L. H. 43; die Mutter war nicht sehr energisch behandelt worden. Im Falle Nr. 69 war eine positive W. R. das einzige Symptom des Kindes; die Mutter hatte drei 
Injektionskuren bekommen; der weitere Verlauf für das Kind ist sehr günstig gewesen. Im nächsten Falle (Nr. 73) war die Behandlung dieselbe wie im vorigen Falle, der Verlauf dagegen nicht vollständig so gut, da sich die Seroreaktion des $31 / 2$ jährigen Kindes positiv zeigte; während der folgenden zwei Jahre erwies sie sich jedoch konstant negativ. Eine dritte Mutter (Fall Nr. 74) war sehr kräftig behandelt worden und reagierte vor, während und nach dem Partus konstant negativ: das Resultat der Schwangerschaft war auch ein großes und symptomfreies Kind, mit negativer W. R. Ähnlich waren auch die Verhältnisse im Falle Nr. 75.

II c) Infektion vor 3 bis 4 Jahren.

2 Mütter waren symptomfrei; die eine (Fall Nr. 76) hatte vor der Schwangerschaft 6 Schmierkuren bekommen, aber während derselben war sie nicht behandelt worden; das Kind hatte klinische Symptome und ergab positive Reaktion noch 5 Jahre alt; später doch negative W. R. Im anderen Falle (Nr. 77) resultierte auch ein syphilitisches Kind mit langer Zeit, trotz spezifischer Behandlung, positiver W. R. Diese Mutter war jedoch sehr energisch behandelt worden - vor der Schwangerschaft eine $\mathrm{Hg}$-S-Kur +4 Injektionskuren und während derselben eine Injektionskur. Sie hatte zwei Jahre vorher auch eine Geburt gehabt (Fall Nr. 39 und L. H. 36). Sie ergab kurze Zeit nach dem letzten Partus noch positive W. R. Eine Mutter, welche weder vor noch während der Schwangerschaft spezifisch behandelt wurde (Fall Nr. 78) und ausgebreitete luetische Symptome darbot, gebar selbstverständlich ein luetisches Kind; nach drei Jahren reagierte es noch positiv. Der vierte Fall (Nr. 79) betrifft eine Mutter, welche vor der Schwangerschaft 3 Hg-S-Kuren + 4 Mk., aber während derselben nichts bekommen batte, drei Monate nach der Entbindung syphilitische Hauterscheinungen und positive W. R. darbot; zu dieser Zeit hatte auch das symptomfrei geborene Kind Syphilissymptome bekommen. 
In dieser Gruppe habe ich zehn Fälle (Nr. 80-89), welche einander vollständig ähnlich sind, zusammengestellt. Alle Mütter hatten ausgeprägte Syphilissymptome. Eine Infektion war in allen Fällen unbekannt und ließ sich durch die Anamnese nicht eruieren. Sie hatten jedoch alle ein Symptom, Leukoderma, wodurch das Alter der maternen Syphilis ungefähr bestimmt werden konnte. Dies Symptom erscheint, wie allbekannt, frühestens ein halbes Jahr nach der Ansteckung und ist in der Regel nach höchstens 4 Jahren verschwunden, dies nach den Angaben in den meisten Hand- und Lehrbüchern (F o urnier, Lesser, Lang u. a.). Daß man auch bei älteren Syphilitikern ein Leukoderma antreffen kann, ist ja jedem Syphilidologen wohlbekannt, aber muß als eine Ausnahme betrachtet werden. Sicherlich gehören diese in Rede stehenden Fälle einer der oben besprochenen Altersgruppen an, aber da die genaue Infektionszeit nicht festzustellen ist, habe ich dieselben als eine Gruppe für sich zusammengestellt.

Wie haben sich die Schicksale dieser kongenitalluetischen Kinder gänzlich unbehandelter Mütter gestaltet? Eigentümlich genug sind sie alle mit Ausnahme der zwei letzten Fälle (Nr. 88-89) im „Lilla Hemmet" aufgenommen worden; dort haben sie sich alle auch unter systematischer Behandlung und sorgfältiger Pflege gut entwickelt; einige haben sich später eingestellt (das älteste von ihnen war damals neun Jahre alt); alle waren anscheinend gesund und die serologisch Untersuchten hatten auch negative W. R. Dies Resultat scheint ja ein bißchen überraschend. Für die Abkömmlinge von Müttern, die bei unbehandelter florider Lues gebaren, wäre ja ein ganz anderes und trauriges Schicksal erfahrungsgemäß zu erwarten; vielleicht frühzeitiger Tod oder wenigstens Rezidive ihrer Krankheit. Aber sowas ist in keinem Falle eingetroffen. Indessen fallen die ersten Lebensjahre der acht ältesten Kinder in den Zeitraum vor der Wassermann-Neisser-Bruckschen Entdeckung; das neunte aber, 1910 geboren, erwies sich bei der serologischen Untersuchung hartnäckig positiv (L. H. 48). Es scheint mir also 
nicht zu kühn zu vermuten, daß auch die älteren kleinen Patienten sich ähnlich verhalten hätten, wenn wir damals eine Seroreaktion zur Verfügung gehabt hätten. Zahlreiche Beispiele dieser Verhältnisse befinden sich ja in meiner Kasuistik, auf welche ich hinweise.

Auch der zehnte Fall (Nr. 89) erwies eine positive W. R., welche die Behandlung nicht verändern konnte.

II $e$ ) Infektion älter als 4 Jahre.

Die vierzehn Fälle, welche ich hier zusammengeführt habe, haben das gemeinsam, daß die Mütter das Sekundärstadium hinter sich hatten: das Alter der mütterlichen Lues schwankt zwischen 4 und 26 Jahren; die Ansteckung läßt sich in allen Fällen durch die Anamnese ziemlich genau feststellen.

Zuerst werde ich drei Fälle, die niemals behandelt waren, besprechen. Zwei Mütter waren symptomfrei, die eine (Fall Nr. 90) hatte negative, die zweite (Fall Nr. 91) positive W. R.; beide Kinder manifeste Symptome und positive W. R.; die Reaktion des letzten Falles erwiès sich jahrelang hartnäckig. Im dritten Falle (Nr. 92) lag die Ansteckung so lange zurück wie 26 Jahre; die Mutter hatte ausgebreitete und schwere tertiäre Erscheinungen und positive W. R.; das Kind manifeste Lues und W. R. +++ .

Die nächsten acht Fälle hatten zwar antiluetische Behandlungen durchgemacht, aber während der Gravidität nicht. Alle diese Kinder hatten positive Seroreaktion, nur eins (Fall 93) war frei von klinischen Lueserscheinungen. Nicht weniger als sechs von ibnengingen in den ersten Lebensmonaten zu grunde. (Fälle Nr. 93-98.)

Das Alter der mütterlichen Syphilis variierte von 4 bis 24 Jahre. Was die Symptome der Mütter betrifft, sei, was von höchstem Interesse ist, zu erwähnen, daß eine Frau an Lues cerebri (Fall 93), eine zweite an Tabes (Fall 97) litten. Fall 95 bietet besonderes Interesse dar. Die Schwangerschaft endete einen Monat zu früh mit einem syphilitischen Kinde mit posi- 
tiver W. R., welches nach 15 Tagen starb; die Mutter hatte während der letzten zwei Jahre kein $\mathrm{Hg}$ bekommen. Sie hatte vorher zwei Kinder gehabt; das erste Kind, postkonzeptionell angesteckt, wurde symptomenfrei und ausgetragen geboren während der Gravidität wurde eine Hg-Kur gegeben. Nachher hat es 3 Säckchenkuren erhalten (also zu wenig), soll keine luetischen Symptome gezeigt haben, hatte aber 4 Jahre alt absolut positive W. R. Das zweite Kind, im achten Graviditätsmonat geboren, starb nach einem Monate; die letzte Behandlung der Mutter lag damals 1/2 Jahre zurïck.

Ich gehe jetzt zu den vier übrigen. Fällen dieser Gruppe über. Alle Mütter waren sowohl vor wie wäbrend der Gravidität spezifisch behandelt; drei waren symptomenfrei; eine (Fall 100) hatte Anzeichen einer ziemlich schweren Syphilis, war nicht besonders kräftig behandelt und gebar ein zwar symptomfreies Kind, das aber trotz kontinuierlicher Hg-Behandlung noch 5 Jahre positiv reagierte: jetzt ist jedoch die Reaktion negativ geworden. Im Falle Nr. 101 war die Behandlung auch nicht besonders kräftig gewesen, trotzdem wurde das Kind symptomfrei und mit negativer Reaktion geboren; nach vier Monaten konnte ich jedoch eine geringe Hemmung der Hämolyse bei der W. R. feststellen. Viel kräftiger war die Mutter im Falle Nr. 102 behandelt; das Kind, symptomfrei geboren, durch Jahre beobachtet, hat sich tadellos entwickelt:

\section{Gruppe III.}

\section{Infektion zeitlich nicht bekannt.}

Hier werden diejenigen Fälle behandelt, wo die Mutter keine Angaben über die Ansteckung geben konnte oder wollte, nebst denjenigen, wo von den Eltern nichts bekannt war. Nur in zwei Fällen war eine Behandlung erfolgt. Im Falle Nr. 104 hatte die Mutter zwar Symptome einer sekundären Syphilis, aber 
der genaue Zeitpunkt der Infektion konnte durch diese nicht bestimmt werden. Sie bekam während der Sohwangerschaft 0.5 Salvarsan; das Kind wurde mit luetischen Erscheinungen geboren, hatte aber negative $W$. $R$, welche jetzt $1 \frac{1}{\mathbf{q}}$ Jahre sich konstant so verhalten hat. Im Falle Nr. 105 wird von der Mutter nur angegeben, daß sie sichere syphilitische Symptome gezeigt haben soll, daß sie eine - näher nicht angegebene - Hg-Kur während der Schwangerschaft bekommen hatte, und daß sie bei der Geburt des Kindes symptomfrei war; das Kind war auch frei von Erscheinungen geboren, starb aber acht Monate alt an Gastroenteritis.

In allen den folgenden Fällen, wo überhaupt einige Angaben betreffs der Mutter zur Verfügung stehen, war die syphilitische Infektion unbekannt und demnach keine Behandlung gegeben. Drei Mütter hatten klinische syphilitische Symptome, im Falle Nr. 106 zeigte der Säugling ausgebreitete solche; zwei Jahre alt an Pneumonie gestorben. Im nächsten Falle (Nr. 107) handelte es sich um eine Frau mit sekundären Symptomen und positiver W. R. Eigentümlich genug wurde das Kind erscheinungsfrei und mit negativer W. R. geboren. Leider wurde der Patient auf Wunsch der Mutter schon nach einigen Wochen entlassen, und die weiteren Schicksale des Kindes sind mir nicht bekannt. In einem späteren Stadium der Syphilis befand sich die Mutter im Falle Nr. 108: von ihr wird nämlich angegeben, daß sie Lues cerebri hatte. Das Kind wurde indessen symptomfrei geboren, hatte aber noch $1 \%$ Jahre alt positive W. R., welche jedoch nachher konstant negativ gewesen ist.

Die folgenden acht Fälle betreffen Mütter, welche nach der Entbindung keine klinischen Symptome hatten; sie gaben jedoch alle positive W. R. In zwei Fällen (Nr. 109, 110) wird angegeben, daß totgeborene oder in jungem Alter gestorbene Kinder vorgekommen sind. Die beiden jetzt in Frage stehenden Kinder kamen syphilitisch zur Welt und sind schon nach einigen Wochen zu Grunde gegangen. In beiden Fällen bekam die Mutter energische $\mathrm{Hg}$-Behandlung mit wiederholten Injektionskuren, mit dem Resultate, daß sie beide, aufs neue geschwängert, ausgetragene, symptomfreie, negativ reagierende Kinder gebaren. 
In den folgenden fünf Fällen waren die Kinder syphilitisch geboren. Wie zu erwarten war, batten die kleinen Patienten, welche im "Lilla Hemmet" aufgenommen sind, trotzdem sie keine Rezidive gezeigt haben, lange Zeit hindurch positive W. R., die der Hg-Behandlung nicht wich (Fälle Nr. 111-115). In einem Falle (Nr. 115) starb das schwer syphilitische Kind zehn Tage alt.

In einem Falle (Nr. 116) sind die Angaben sehr ungenau: Die Mutter und eine Schwester soll W. R. +++ haben; der Patient soll während des zweiten Lebensjahres eine Meningitis luetica gehabt haben, die unter Hg-Behandlung schwand; war bei der Aufnahme im "Lilla Hemmet" symptomfrei und gab negative W. R. Er war damals beinahe drei Jahre alt. Schließlich kommen zur Besprechung diejenigen $10 \mathrm{Kinder}$, von deren Müttern keine Angaben aufzubringen waren. Unter solchen Umständen sind diese Fälle selbstverständlich von relativ geringem Wert, um Beiträge zur Beleuchtung des Themas dieser Arbeit zu leisten, und ich verweise betreffs derselben auf die Kasuistik. Zwei Kinder (Fälle Nr. 125, 126) sind schon in den ersten Lebensmonaten und eins (Fall Nr. 127) neun Monate alt gestorben.

Ich gehe jetzt dazu über, die oben angeführten Beobachtungen summarisch zu verwerten. Der Übersicht halber habe ich die hierbei gewonnenen Ziffern in Tabellen nach d e m Al ter d er maternen Syphilis, nach den Erscheinungen der $M \ddot{u} t$ ter und nach der gegebenen Behandlung aufgestellt. Doch scheint es mir nötig, zuvor eine kleine Bemerkung zu machen.

Gegen die angewandte Bezeichnung "Frei von Erscheinungen" könnte vielleicht die Einwendung gemacht werden, daß unter den so bezeichneten Patienten mehrere sich befinden, welche vor der Wassermannschen Ära beobachtet waren und demnach nicht sicher als symptomfrei anzusehen wären. Diese Anmerkung ist vollständig berechtigt, aber bedeutet bei den Müttern nichts, weil die Erscheinungen der Mütter nur wenig Einfluß auf das neugeborene Kind haben (vide Tabelle II). Viel wichtiger ist dagegen diese Fehlerquelle, wenn wir daran gehen, den Zustand des neugeborenen Kindes zu beurteilen. Glücklicherweise ist die durch dieselben hervorgerufene 
Unsicherheit von sehr geringer Bedeutung, wie ich gleich zeigen werde.

Von den 114 untersuchten Säuglingen waren 4] klinisch symptomfrei; von diesen waren 25 serologisch untersucht; 9 hatten positive W. R. $(=36 \%)$ und sind demnach in den Tabellen als mit Erscheinungen behaftet behandelt. Nur 16 gehören der Zeit vor der Wassermannschen Entdeckung an; wenn wir hier mit dem jetzt angegebenen Prozente rechnen, wäre eventuell 5.8 von diesen Kindern als nicht erscheinungsfrei zu bezeichnen. Das bedeutet bei der Totalmenge von 114 Säuglingen (Tabelle I) eine Vermehrung der sicher syphilitischen Geburten von 82 bis 87.8 , d. i. eine Verschiebung des Prozentes von 71.9 bis 77. Dieser Unterschied ist indessen allzu klein, um der Fehlerquelle eine we sentliche Bedeutung zuzumessen.

Ich gehe zur Besprechung der Tabellen über.

Tabelle I.

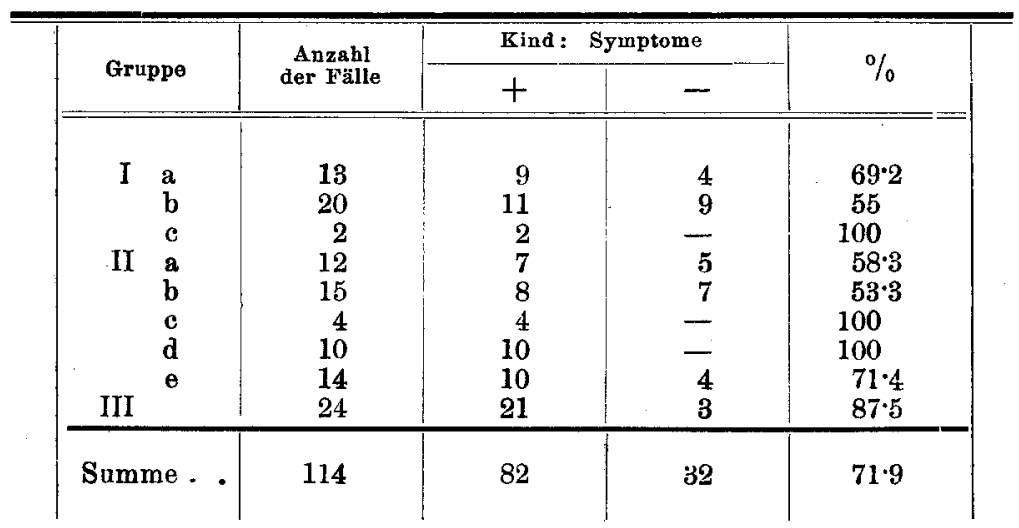

Die Zahlen der einzelnen Altersgruppen sind ja allzu klein, um bestimmte Schlüsse ziehen zu können. So viel erhellt jedoch, daß das Alter der mütterlichen Lues keinen entscheidenden EinfluB auf den Zustand des Säuglings haben kann. Die niedrigsten Prozentziffern sehen wir ja sogar bei den Gruppen I b, II a und b, welche doch lauter Fälle mit sekundärer Syphilis umfassen. Die Erklärung liegt natürlich darin, daß diese Patienten in der Regel unter Behandlung stehen, im Gegensatz zu den älteren Gruppen, 
wo oft viele Jahre seit der letzten Kur vergangen sind. (Siehe weiter Tabelle III.)

Tabelle II.

\begin{tabular}{|c|c|c|c|c|c|c|c|c|}
\hline \multirow{5}{*}{ Gruppe } & \multicolumn{8}{|c|}{ Mutter } \\
\hline & \multicolumn{4}{|c|}{ mit } & \multicolumn{4}{|c|}{ ohne } \\
\hline & \multicolumn{8}{|c|}{ Erscheinungen } \\
\hline & \multirow{2}{*}{$\begin{array}{c}\text { Anzahl } \\
\text { Falle }\end{array}$} & \multicolumn{2}{|c|}{$\begin{array}{c}\text { Kind: } \\
\text { Symptome }\end{array}$} & \multirow{2}{*}{$\begin{array}{l}\% \\
+\end{array}$} & \multirow{2}{*}{$\begin{array}{c}\text { Anzehl } \\
\text { Falle }\end{array}$} & \multicolumn{2}{|c|}{$\begin{array}{c}\text { Kind: } \\
\text { Symptome }\end{array}$} & \multirow{2}{*}{$\begin{array}{l}\% \\
-\end{array}$} \\
\hline & & + & - & & & + & - & \\
\hline I a & 13 & 9 & 4 & $69 \cdot 2$ & . & . & - & . \\
\hline b & 20 & 11 & 9 & 55 & • & . & . & • \\
\hline e & 2 & 2 & . & 100 & . & . & . & . \\
\hline II a & 12 & 7 & 5 & $58 \cdot 3$ & . & . & . & \\
\hline b & 8 & 4 & 4 & 50 & 7 & 4 & 3 & $57 \cdot 1$ \\
\hline c & 2 & 2 & . & 100 & 2 & 2 & . & 100 \\
\hline$d$ & 10 & 10 & . & 100 & & & $\cdot$ & \\
\hline & 6 & 4 & 2 & $66 \cdot 7$ & 8 & 6 & 2 & 75 \\
\hline III & 6 & 3 & 3 & 50 & 8 & 8 & . & 100 \\
\hline Summe . & 79 & 52 & 27 & $65 \cdot 8$ & 25 & 20 & 5 & $\left.80^{1}\right)$ \\
\hline
\end{tabular}

Die Erscheinungen der Miitter spielen auch keine Rolle für die Neugeborenen. Symptomfreie Mütter gebaren sogar $80 \%$ sicher syphilitische Kinder, während die Zahl für die mit Erscheinungen behafteten $65.8 \%$ war.

Tabelle III.

\begin{tabular}{|c|c|c|c|c|c|c|c|c|c|c|c|c|}
\hline \multirow{3}{*}{$\begin{array}{l}\text { 品 } \\
\text { 胥 }\end{array}$} & \multicolumn{4}{|c|}{$\underset{\text { anbehandelt }}{\text { Muter }}$} & \multicolumn{4}{|c|}{$\begin{array}{l}\text { Behandlung, } \\
\text { aber nicht während der } \\
\text { Schwangersehaft }\end{array}$} & \multicolumn{4}{|c|}{$\begin{array}{c}\text { Behandlung } \\
\text { während der } \\
\text { Sehwangersehaft }\end{array}$} \\
\hline & \multirow{2}{*}{$\begin{array}{l}\text { Anzabl } \\
\text { Fälle }\end{array}$} & \multicolumn{2}{|c|}{$\begin{array}{l}\text { Kind: } \\
\text { Sympt. }\end{array}$} & \multirow{2}{*}{$\begin{array}{l}\% \\
+\end{array}$} & \multirow{2}{*}{$\begin{array}{c}\text { Anzahl } \\
\text { Fălle }\end{array}$} & \multicolumn{2}{|c|}{$\begin{array}{l}\text { Kind: } \\
\text { Sympt. }\end{array}$} & \multirow{2}{*}{$\begin{array}{l}\% \\
+\end{array}$} & \multirow{2}{*}{$\begin{array}{c}\text { Anzahl } \\
\text { Fälle }\end{array}$} & \multicolumn{2}{|c|}{$\begin{array}{l}\text { Kind: } \\
\text { Sympt. }\end{array}$} & \multirow{2}{*}{$\begin{array}{l}\% \\
+\end{array}$} \\
\hline & & + & - & & & + & - & & & + & - & \\
\hline & 6 & 5 & 1 & $83 \cdot 3$ & - & - & . & - & 7 & 4 & 3 & $57 \cdot 1$ \\
\hline & 7 & 6 & 1 & $85 \cdot 7$ & - & . & . & . & 13 & 5 & 8 & $\mathbf{3 8} \mathbf{5}$ \\
\hline & & & • & & & & . & & 2 & 2 & & 100 \\
\hline II $\mathbf{a}$ & 2 & 2 & . & 100 & 2 & 2 & . & 100 & 8 & $\overline{3}$ & 5 & $37 \cdot 5$ \\
\hline & 1 & 1 & . & 100 & 4 & 2 & 2 & 50 & 10 & 5 & 5 & 50 \\
\hline & & & . & & 3 & 3 & . & 100 & 1 & 1 & . & 100 \\
\hline & 10 & 10 & . & 100 & & $\dot{\bullet}$ & . & & $\dot{*}$ & - & . & • \\
\hline & $\mathbf{3}$ & 3 & . & 100 & 8 & 7 & 1 & $87 \cdot 5$ & 3 & . & 3 & \\
\hline III & 12 & 10 & 2 & $83 \cdot 3$ & - & . & . & . & 2 & 1 & 1 & 50 \\
\hline Summe & 41 & 37 & 4 & $90 \cdot 2$ & 17 & 14 & 3 & $82 \cdot 3$ & 46 & 21 & 25 & $\left.45^{\cdot} 6^{1}\right)$ \\
\hline
\end{tabular}

1) 10 Fälle ohne jegliche Angaben betreffs der Mutter sind hier nicht mitgerechnet. 
Die durch die Erfahrung wohlbekannte segensreiche Wirkung der intrauterinen Behandlung wird durch die hier angeführten Zahlen kräftig bestätigt.

Unbehandelte Mütter gebaren $90.2 \%$, nicht während der Schwangerschaft behandelte $82 \cdot 3 \%$, während der Gravidität behandelte nur $45.6 \%$ sicher syphilitische Kinder!

Tabelle IV.

\begin{tabular}{|c|c|c|c|c|c|c|c|c|c|c|c|c|c|c|c|}
\hline \multirow{2}{*}{ 总 } & \multirow{2}{*}{ 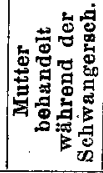 } & \multirow{2}{*}{ 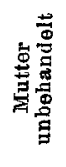 } & \multicolumn{12}{|c|}{ Lebensmonat } & \multirow{2}{*}{ 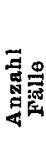 } \\
\hline & & & 1 & 2 & $\mathbf{3}$ & 4 & 5 & 6 & 7 & 8 & 9 & 10 & 11 & 12 & \\
\hline 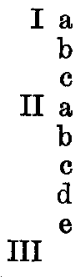 & $\begin{array}{l}2 \\
2 \\
1 \\
3 \\
1 \\
.\end{array}$ & $\begin{array}{l}1 \\
3 \\
\cdot \\
\cdot \\
: \\
\cdot \\
\dot{5} \\
5\end{array}$ & $\begin{array}{l}2 \\
1 \\
1 \\
2 \\
. \\
\dot{1} \\
1 \\
2\end{array}$ & $\begin{array}{l}1 \\
\mathbf{1} \\
\dot{ } \\
\dot{ }\end{array}$ & $\begin{array}{l}1 \\
\dot{.}\end{array}$ & $\begin{array}{l}1 \\
\text { i } \\
\dot{1} \\
\text { i } \\
1\end{array}$ & $\begin{array}{l}1 \\
\text {. } \\
\text {. }\end{array}$ & $\begin{array}{l}1 \\
\dot{5} \\
\dot{.} \\
\dot{.} \\
\dot{.}\end{array}$ & : & : & $\dot{:}$ & : & $\dot{5}$. & $\begin{array}{l}\dot{5} \\
\dot{5} \\
\dot{5}\end{array}$ & $\begin{array}{l}3 \\
5 \\
1 \\
3 \\
1 \\
\cdot \\
6 \\
6 \\
5\end{array}$ \\
\hline Summe & 10 & 14 & 9 & 3 & 3 & 4 & 2 & 1 & - & . & 1 & . & 1 & . & 24 \\
\hline
\end{tabular}

24 Kinder von den 114 sind während des ersten Lebensjahres gestorben, d. i. eine Mortalität von $21 \%$. 10 Mütter waren während der Schwangerschaft behandelt, ke in e e in zige jed och wirklich energis ch; vier hatten Salvarsan bekommen, eine III. (Fall 21), eine II. + eine Hektinekur (Fall 49) und zwei nur eine Injektion (Fälle 48 und 64). Im Falle 50 hatte die Mutter nur eine Hektinekur und $2 \mathrm{Mk}$. bekommen. Alle die übrigen 14 waren unbehandelt.

Nicht weniger als 22 Kinder sind im ersten Halbjahre gestorben, und 9 von diesen sind schon im ersten Lebensmonate zu grunde gegangen.

Die Todesursachen waren: 


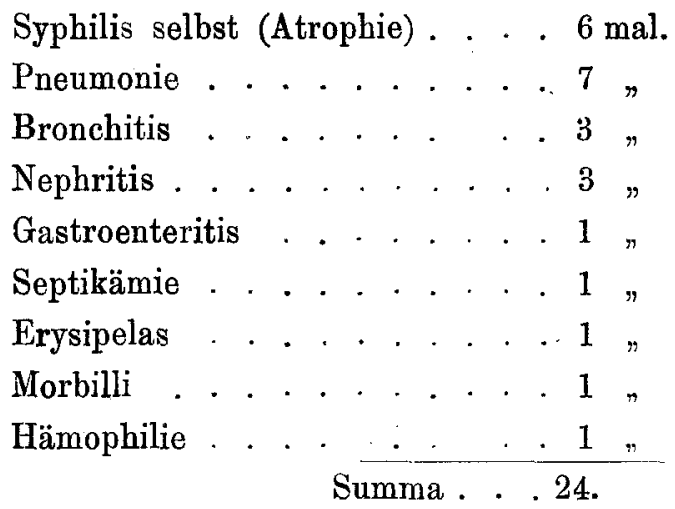

Das Mortalitätsprozent ist relativ sehr gering, wenn man bedenkt, daß die Mehrzahl der Säuglinge unter die Frühgeburten zu rechnen sind; zufolge der in einigen Fällen unvollständigen Angaben sehe ich jedoch von einer näheren Berechnung hier ab.

Die Todesursachen sind die gewöhnlichen und stimmen mit dem, was wir von größeren Statistiken schon wissen.

Der Grundsatz, bei einer frischen Syphilisinfektion so schnell wie möglich mit spezifischer Behandlung einzugreifen, hat, wie aus obigem hervorgeht, bei den luetischen Schwangerschaften die Prozentzahl symptomfreier Kinder von $9.8 \%$ bis 54.4 erhöht! Aber $45.6 \%$ luetische Früchte haben wir noch zu verzeichnen, und von den symptomfrei Geborenen bekommen viele später Erscheinungen! Das einzige Mittel, diese kleinen Kinder zu retten, ist selbstverständlich nur die fortgesetzte spezifische Behandlung, und nicht nur eine oder zwei Kuren, sondern systematisch und während mehrerer Jahre wiederholte Kuren. Unter günstigen äußeren Bedingungen läßt sich dies auch meistens leicht tun, aber für die ärmere Bevölkerung wird es wohl in der Regel nicht gelingen, besonders weil die Mehrzahl der syphilitischen Kinder 
unehelich geboren sind, mit allen den damit drohenden Folgen sowohl für ihre eigene Pflege, wie für ihre zufällige Umgebung. Die Rettung vor dem fürchterlichen Unheile eines solchen Daseins suchte nun Edward Welander durch Einrichtung eines Asyles zu finden, wo die kongenitalluetischen Kinder 3-4 Jahre bleiben könnten. Eine solche Anstalt wurde auch, wie bekannt, von ihm 1900 in Stockholm eröffnet. Die Erwartungen, welche an das "Lilla Hemmet" geknüpft waren, sind im höchsten Grade erfüllt worden, den kleinen Patienten zum Besten und dem großen Gedanken des Urhebers zur unvergänglichen Ehre!

Das Alter der kleinen Patienten bei der Aufnahme ist durchschnittlich $1 / 2-1 \frac{1}{2}$ Jahre gewesen. Im allgemeinen wúrden sie zuerst in St. Göran mehrere Monate gepflegt, um die eventuellen ersten ansteckenden Erscheinungen unter der $\mathrm{Hg}-$ Behandlung zur Heilung zu bringen und so viele Säuglinge wie möglich so lange wie möglich an der Mutterbrust zu halten. Nur drei Kinder - 30, 53 und 54 - sind mit 2 Jahren 4 Monaten, 2 Jahren 5 Monaten und 3 Jahren 10 Monaten aufgenommen worden, und dies aus äußeren Gründen. 53 hatte vor der Aufnahme Gummen des Gesichtes und 54 eine luetische Meningitis gehabt; beide waren unter $\mathrm{Hg}$-Behandlung erscheinungsfrei geworden; 54 hatte negative W. R., 53 dagegen hartnäckig positive, nebst Narben an der Stirn und tiefe radiäre Narben um den Mund.

Alle Patienten des "Lilla Hemmet" werden chronisch intermittierend behandelt. Die Methode ist die von Welander empfohlene Säckchenmethode, welche sich für die jetzt in Frage kommenden kleinen Patienten außerordentlich zweckmäßig gezeigt hat: kräftige therapeutische Wirkung, keine Hautreizung wie bei der Schmierkur, keine Verdauungsstörungen wie bei der internen Darreichung des Hg. Die Dosen sind $0.5-2 g$ täglich gewesen und die Kuren haben als Regel 30-40 Tage gedanert. Schädliche Nebenwirkungen des $\mathrm{Hg}$ sind nur ausnahmsweise und in Form von leicht vorübergehender Albuminurie und zufälliger Gewichtsabnahme vorgekommen. Harnuntersuchungen wurden systematisch vorgenommen; nachdem 
die $\mathrm{W}$ a s s er m a $\mathrm{n} n$ sche Seroreaktion zur Anwendung gekommen ist, ist dieselbe jeden dritten Monat vorgenommen worden.

Vier Patienten sind mit Salvarsan behandelt worden. Nr. 41 und $43,2 \frac{1}{2}$ und $1 \frac{1}{2}$ Jahre alt, welche bei wiederholten Untersuchungen positiv reagiert hatten, bekamen $0.043 \mathrm{~g}$ „606" intramuskulär; eine deutliche Besserung war während des nächsten Jahres nicht festzustellen. Die kleinen Kinder hatten noch beinabe $2 \frac{1}{4}$ Jahre nach der Injektion pflaumengroße Infiltrate in den Glutäen. Nr. 48 bekam am 5./IX. 1910, vier Monate alt, $0.03 g$ Hata intramuskulär und Nr. 57 erhielt, einen Monat alt, $0.02 \mathrm{~g}$ Salvarsan, nach drei Monaten weitere $0.035 \mathrm{~g}$. Zwei Monate später Rezidiv und bei der Aufnahme ein-halbes Jahr nachher im "Lilla Hemmet" hatte sie ausgebreitete syphilitische Erscheinungen. In allen den mit Salvarsan behandelten Fällen erwies sich, wie aus der Übersicht hervorgeht, die Wassermannsche Reaktion als außerordentlich hartnäckig positiv.

Von den bisher in „Lilla Hemmet“ gepflegten 69 kongenitalluetischen Kindern sind acht während des Aufenthaltes im Asyl gestorben; ein Kind (Nr. 19) ist während einer Diphtherieepidemie, welche das Asyl während des Jahres 1904 heimsuchte, im Isolierungskrankenhause gestorben. Die übrigen sieben erlagen denjenigen Krankheiten, welche die kongenitalluetischen Kinder am öftesten töten, drei $(5,6,18)$ an Preumonie, zwei $(36,59)$ an Bronchitis und zwei $(7.38)$ an Gastroenteritis. Rechnen wir das an Diphtherie gestorbene Kind, was mir richtig scheint, weg, so haben wir also sieben Todesfälle zu verzeichnen; das ist eine Mortalität von $10 \cdot 14 \%$.

Zum Vergleich will ich entsprechende Ziffern aus jüngst veröffentlichten Statistiken anführen: Hochsinger gibt an, daß ron 263 lebendgeborenen Luetikern $55(29 \cdot 9 \%)$ starben vor Vollendung des vierten Lebensjahres, trotzdem mehrere von ihnen spezifische „Frühbehandlung" erhielten. Reuben endlich sah $\mathbf{7 5 \%}$ aller Früchte syphilitischer Mütter vor Vollendung des ersten Lebensjahres sterben!

Nicht weniger als sechs von unseren Todesfällen gehören der Periode an, wo das "Lilla Hemmet" provisorisch in einem alten 
Gebäude, wo die nötigen hygienischen Maßnahmen nicht durchzuführen waren, beherbergt war; seitdem die kleinen Patienten das vor zwei Jahren eingeweihte, hygienisch außerordentlich gut eingerichtete neue Heim in Besitz genommen haben, ist nur ein Todesfall eingetroffen; die Patientin (59) war sehr schwächlich gewesen, atrophisch geboren; die Mutter sehr wenig behandelt, nicht während der Gravidität. Bei keiner der vorgenommenen Sektionen konnten luetische Veränderungen beobachtet werden.

Fin Kind (24), das während des Aufenthaltes im Asyle keine syphilitischen Erscheinungen gezeigt batte, war schon im vierten Lebensjahre an chronischer Bronchitis leidend, und starb $6 \frac{1}{2}$ Jahre alt in einem Kinderspitale an Pneumonie; einige Monate vorher hatte es bei der Untersuchung keine klinischen Erscheinungen und negative W. R. Diesen Fall rechne ich aus diesen Gründen nicht unter die Todesfälle, welche das Mortalitätsprozent des "Lilla Hemmet" bestimmen, sondern habe ihn unter die günstig verlaufenen Fälle gerechnet. (Beobachtungszeit 61/2 Jahre.) Ebenso ist der Fall 2 (sechs Jahre alt durch Unfall gestorben; kurz vorher bei Untersuchung keine Erscheinungen) behandelt worden.

31 Kinder sind nach durchschnittlich mehr als drei Jahren entlassen; von fünf haben wir keine weiteren Mitteilungen bekommen; von den übrigen 26 dagegen wissen wir soviel, daß wir ein Urteil über ihre Schicksale geben können. Die Kinder werden an verschiedenen Orten, teils durch die Behörde, teils durch private Maßnahmen am meisten in Familien aufgenommen; an diese wurden im Mai 1912 schriftliche Fragen über den Zustand des Patienten gerichtet; die Fragen sind selbstverständlich mit Hinsicht auf die gewöhnlichen Stigmata, die körperliche und geistige Entwicklung und eventuelle akute Infektionskrankheiten aufgestellt. Auf die eingelaufenen Antworten und auf eigene Beobachtungen der in Stockholm wohnenden Kinder sind die Urteile gestützt, die jetzt mitgeteilt werden sollen.

Die Beobachtungszeit war in

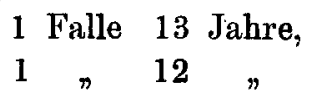




$\begin{array}{llll}3 & \text { Fällen } 11 & \text { Jahre } \\ 1 & \text { Falle } & 10 & n \\ 4 & \text { Fällen } & 9 & n \\ 2 & n & 8 & " \\ 6 & n & 7 & n \\ 2 & \# & 6 & " \\ 5 & n & 5 & n\end{array}$

Ein Fall (54) wird auf Grund gewisser Umstände (siehe oben) hier nicht mitgerechnet.

Nur ein einziges Kind (13) hat ein sicheres Syphilisrezidiv gehabt. Die Mutter wußte nichts von einer syphilitischen Infektion und war bei der Untersuchung symptomfrei. Die Patientin soll während des ersten Lebensjahres nichts abnormes gezeigt haben. Mit einem Jahre Ausschlag am After. Keine antiluetische Behandlung. Drei Monate später, im Krankenhause St. Göran aufgenommen, hatte sie muköse Papeln ad anum, Erosionen an den Tonsillen und Inguinal- und Zervikaladenitis. Nach $40 \mathrm{Hg}$-S. à $1 \mathrm{~g}$ waren die Erscheinungen verschwunden. Während der nächsten drei Jahre wurde sie im "Lilla Hemmet" präventiv intermittent mit Säckchenkuren behandelt und war die ganze Zeit erscheinungsfrei. Nach der Entlassung wurde sie durch die Vermittlung der allgemeinen Waisenanstalt zu Stockholm von einer Familie aufgenommen. Fünf Jahre alt wurde sie in die Krankenabteilung dieser Waisenanstalt wegen einer Augenerkrankung aufgenommen. Aus dem Journale mag folgendes angefuihrt werden. Graziler Körperbau; gesundes Aussehen; Kopf wohlgebildet; Gehör gut; ein oberer Schneidezahn an der Unterseite ein bißchen ausgehöhlt; diffuse Trübung der linken Hornhaut, in der Mitte am meisten gesättigt; Perikornealinjektion; die Pupille reagiert auf Licht. Das rechte Auge gesund, aber an diesem traten nach einem Monate ähnliche Veränderungen wie am linken auf. Nach Schmierkur ( $21 / 2 g$ Tage) heilte die Augenkrankheit aus. Ein Jahr später konnten bei der Untersuchung kaum Spuren der parenchymatösen Keratitis entdeckt werden; im übrigen war sie vollständig erscheinungsfrei. Nach drei Jahren wurde sie, wegen einer unbedeutenden Verletzung durch Unfall, wieder in der Waisenanstalt aufgenommen; damals war sie völlig er- 
Klin. Beobachtungen über die Prognose der kong. Syphilis.

scheinungsfrei. Stat. praes. 28./VI. 1912. Graziler Körperbau. Hautfarbe etwas blaß. Am Kinn und am Halse zwei lineäre Narben (nach dem oben erwähnten Unfalle); in diesen sind derbe Keloide entstanden. Beide Korneae jetzt vollständig klar; Sehvermögen gut. Gehör auch. Keine anderen Zeichen, welche mit ihrer Lues in Zusammenhang zu setzen wären. Geht in die Schule; soll nicht unter die besten Schüler zu rechnen sein, aber ist nicht als zurückgeblieben zu betrachten. W. R. negativ.

Die enorme Bedeutung der friihzeitigen, d. h. intrauterinen Behandlung geht aus den Auseinandersetzungen im vorigen Abschnitte dieser Arbeit unzweideutig hervor. Eine solche Behandlung war in diesem Falle nicht gegeben worden und es dauerte ja $1 \frac{1 / 3}{3} \mathrm{Jahr}$, bis das Kind $\mathrm{Hg}$ bekam. Daß in einem solchen Falle die nachfolgende kräftige $\mathrm{Hg}$-Behandlung die $\mathrm{Pa}-$ tientin gegen ein Rezidiv nicht schützen konnte, ist ja nicht zu verwundern. Einige Fälle, welche ähnliche Verhältnisse mit Hinsicht des zu späten Einsetzens der Behandlung aufweisen, sind Nr. 53, 62, 63 und 66. (Siehe die tabellarische Übersicht.)

Die Fälle $(22,35,37)$ haben, wie aus der Übersicht hervorgeht, solche Erscheinungen dargeboten, welche auf einer syphilitischen Infektion beruhen $k$ önnen aber nicht $m$ ü ssen. In keinem dieser Fälle ist eine intrauterine Behandlung gegeben worden. Im Falle 22 ist eine serologische Untersuchung nicht möglich gewesen, in den zwei übrigen Fällen wurde die Seroreaktion bei wiederholten Untersuchungen konstant negativ befunden. Dies spricht selbstrerständlich gegen die Annahme, daß die bej den beiden Kindern beobachtete, etwas mangelnde geistige Entwicklung auf ihre Syphilis zurückzuführen sei. In einem Falle lag Alkoholismus der Mutter vor.

Wir sehen also, daß nur ein Kind von den 26 in Frage stehenden ein sicheres Rezidiv bekommen bat $(3 \cdot 8 \%)$. Nach den Angaben von Sprinz sah $\mathrm{P}$ eiser Rezidive in ca. $1 / 3$ der Fälle, Hochsinger dagegen bei $63 \%$ der von ihm behandelten Kinder.

Dies, man kann wohl sagen, glänzende Resultat des nLilla Hemmet" ist wohl darauf zurückzuführen, daß die intrauterine und die chronisch intermittente Behandlung bei den meisten 
der Patienten zielbewußt angewendet wurde. In zehn von den gelungenen Fällen waren die kleinen Patienten schon im Mutterleibe spezifisch behandelt; in allen Fällen wardie Fournier-Neisser-Welandersche Behandlung gegeben worden.

Während des Aufenthaltes im "Lilla Hemmet" also bei einer Periode der kongenitalen Syphilis, welche dem Sekundärstadium der erworbenen Lues entspricht, haben drei Kinder Rezidive gezeigt.

Nr. 41 hatte gegen das Ende des ersten Lebensjahres spezifische Erosionen am After und im Munde und ein Jahr später Papeln an der Stirn; die Mutter, gleichzeitig mit der Konzeption angesteckt, hatte nur eine Kur durchgemacht; der kleine Patient bekam "606". (Siehe oben.)

Nr. 45. Mutter unbehandelt. Bekam im zweiten Lebensjahre Schleimpapeln in der Vulva und an den Tonsillen.

Nr. 28 wird etwas näher besprochen werden. Die Mutter hat drei Schmierkuren durchgemacht; während der Gravidität keine Behandlung. Das Kind bei der Geburt ausgetragen, symptomlos. Drei Wochen alt im Krankenhause St. Göran aufgenommen; blutigen Schnupfen, Leber- und Milzvergrößerung, Ikterus. Flecken und Papeln am Körper, muköse Papeln im Munde und in der Vulva; Atrophie. Nach 2 Hg-Kuren symptomenfrei; sechs Monate alt ins "Lilla Hemmet" aufgenommen. Zwei Jahre vier Monate alt (23./XI. 1907), nachdem sie zusammen $9 \mathrm{Hg}$-Kuren bekommen und keine Erscheinungen gezeigt hatte, wurde sie unruhig, schlaflos, rollte den Kopf und den linken Arm bald nach rechts, bald nach links; Schwierigkeit sich selbst aufzusetzen; kein Fieber. 24./XI. Parese des unteren linken Fazialis, schlaff in den Beinen. 25./XI. Ord. JK. 30./XI. Paralyse des genannten Nerrens; kann etwas gehen, doch parätisch und unsicher; kann sich nicht aufrichten. Im allgemeinen ruhig, rollt sie doch ab und zu den Kopf und den linken Arm, spricht unverwirrt, gibt auf die Umgebung acht. Tuberkulinprobe negativ. Ord. JK. + Hg. 21./X. Paralyse vermindert, Beweglichkeit in den Beinen vermehrt. 12./L 1908 wesentliche Besserung. 10./II. geheilt. Unter fortgesetzter Hg- 
Behandlung hat sie keine neuen Erscheinungen bekommen; gab bei wiederholten Untersuchungen negative W. R.; wurde entlassen und hatte sich $1912 \mathrm{im}$ Juni (also 7 Jahre alt) zu einem geistig und körperlich normalen Kinde entwickelt.

$\mathrm{DaB}$ es sich hier um eine Meningitis luetica handelt, ist wohl fast sicher. Dafür sprechen vor allem die deutlichen Symptome und übrigens das gute Resultat der JK.- und HgBehandlung; gegen eine tuberkulöse Meningitis sprechen die Abwesenheit des Fiebers und die negative Tuberkulinprobe.

Nur drei von den 69 Kindern haben also während der ersten Lebensjahre klinische Rezidive gezeigt, d. i. ein Prozent von $4 \cdot 35$.

In gewissem Maße sind diejenigen Fälle mit den Rezidiven zu vergleichen, welehe, ohne sichtbare Erscheinungen zu haben, nach mehreren negativen Proben eine positive W. R. bekommen, oder eine solche hartnäckig durch Jahre haben, trotzdem die Behandlung die ganze Zeit hindurch so kräftig wie möglich fortgesetzt wurde.

$\mathrm{Zu}$ dieser Gruppe gehören, streng genommen, $18 \mathrm{Kinder}$; von diesen sind indessen vier $(62,63,65$ und 66$)$ noch allzn kurze Zeit (ungefähr ein Jahr) in Beobachtung gewesen, um mit in Betracht zu kommen. Nr. 53 ist ein schwerer Fall. Zuerst 2 Jahre und 4 Monate alt ins Asyl aufgenommen, vorher ungenügend behandelt, und Nr. 57 ist das schon oben erwähnte Mädchen, das, in der Waisenanstalt mit Salvarsan behandelt, vor der Aufnahme ins "Lilla Hemmet“ zwei Rezidiven gezeigt hatte. Dall diese beiden Fälle hartnäckig positive W. R. geben sollten, war ja zu erwarten, und da sie beide, auf Grund der jetzt erwähnten Umstände Ausnahmen von den anderen Patienten machen, rechne ich sie nicht hier mit.

Die 12 übrigen Kinder verhalten sich wie folgt: Ein Knabe (Nr. 32) hatte, noch 5 Jahre alt, W. R. +++ , keine intrauterine Behandlung; nach $\mathrm{Hg}$-Behandlung negative Reaktion. Vier Kinder (26, 27, 30 und 33) reagierten positiv noch vier Jahre alt. In den Fällen 26 und 30 waren die Mütter nicht während der Schwangerschaft behandelt, im Falle 27 nur sehr schwach; das Kind Nr. 30 war bei der Aufnahme 2 Jahre 4 Monate alt. Alle diese Kinder reagierten 
indessen bei späteren Untersuchungen negativ; sie waren selbstverständlich erneuter $\mathrm{Hg}$-Behandlung unterworfen worden. Die Kinder Nr. 43, 48, 50 und $\check{\jmath} 5$ hatten mit 2 Jahren noch positive W. R.; unter fortgesetzter spezifischer Behandlung wurde 43 und 50 im dritten Lebensjahre negativ, währenddem bei den zwei übrigen ein Umschwung der Reaktion noch nicht zu beobachten ist; nur im Falle 43 war die intrauterine Behandlung gegeben, in den anderen nicht; 43 und 50 waren 12 Monate alt und 5511 Monate alt ins "Lilla Hemmet" aufgenommen worden. Schließlich ist zu erwähnen, daß die positive W. R. bei den Kindern Nr. 58 und $601 \frac{1}{2}$ Jahre nach der Geburt noch bestand, und bei dem Knaben Nr. 46 erst nach einem Jahre negativ wurde.

Nur vier Mütter wurden während der Schwangerschaft spezifisch behandelt, die übrigen acht nicht. Fünf Mütter waren serologisch untersucht, alle hatten positive W. R.

Wie aus dem jetzt Angeführten erhellt, sind die scheinbar ungünstigen Resultate der serologischen Untersuchungen in diesen 12 Fällen bei näherer Prüfung doch nicht so schlecht; teils war die intrauterine Behandlung nur in einigen Fällen gegeben und teils sind es ja Kinder, die erst in älteren Jahren ins "Lilla Hemmet" aufgenommen wurden und vorher entweder besonders schwere Symptome gezeigt hatten, oder ungenügende Behandlung bekommen hatten. Ohne Zweifel werden alle die kleinen Patienten, welche noch positiv reagieren, unter fortgesetzter Hg-Behandlung allmählich negativ werden, wie es schon mit den fünf ältesten Kindern geschehen ist.

Neunzehn andere Kinder sind mehrmals serologisch untersucht worden. Davon waren sechs bei der ersten Untersuchung schon lange Zeit antiluetisch behandelt worden; nur ein Kind (Nr. 39) hatte bei der ersten Untersuchung schwach positive W. R. - intrauterine Behandlung war nicht erfolgt; sowohl dieses als die fünf übrigen gaben bei allen folgenden Untersuchungen konstant negative Seroreaktion.

Die übrigen hierhergehörigen 13 Kinder wurden schon als Neugeborene serologisch untersucht. Acht Mütter waren während der Schwangerschaft spezifisch behandelt; nur drei Kinder (Nr. 47, 51, 64) hatten positive Reaktion, welche jedoch schnell 
in eine negative überführt werden konnte. Nicht weniger als fünf Neugeborene reagierten negativ und verhielten sich so bei allen bisher vorgenommenen Untersuchungen (Nr. 44, 52,68,69, 70).

Die Kinder der fünf während der Schwangerschaft unbehandelten Mütter hatten bei der Geburt alle positive W. R., welche in diesen Fällen jedoch ziemlich leicht negativ wurde.

Die serologischen Untersuchungen im „Lilla Hemmet" haben also als Resultat ergeben, daß unter den Kindern, welche sich während längerer Zeit positiv verhielten, nur vier von zwölf $(=25 \%)$ intrauterin behandelt worden waren, während die entsprechenden Zahlen bei den serologisch negativ verlaufenden Fällen elf von neunzehn $(=60 \%)$ waren. Fünf von den acht Neugeborenen, welche intrauterin behandelt worden waren, reagierten von Anfang an negativ; alle die fünf dagegen, die aut diese Weise nicht behandelt worden waren, wurden mit positiver W. R. geboren.

\section{Zusammenfassung.}

Sollte ich jetzt einen Versuch machen, aus dem oben bearbeiteten Materiale einige Schlüsse betreffs der Schicksale der kongenital luetischen Kinder, d. i. der Progn ose, zu ziehen, so wäre vor allem folgendes hervorzuheben:

1. Weder das Alter der maternen Syphilis noch die Erscheinungen der Mütter bei der Entbindung haben einen bestimmten Einfluß auf den Zustand des neugeborenen Kindes gezeigt (Tabelle I und II).

2. Die spezifische Behandlung während der Schwangerschaft hat dagegen einen außerordentlich günstigen Einfluß gezeigt. (Tabelle III.)

3. Eine durch Jahre fortgesetzte Behandlung ist jedoch notwendig, um Rezidive zu verhindern und die Seroreaktion dauernd negativ zu halten. Die Resultate einer solchen, wie sie aus den bisherigen Erfahrungen des "Lilla Hemmet" hervorgehen, sind außerordentlich gute gewesen: ein einziges Rezidiv auf 26 entlassene Kinder (längste Beobachtungszeit 13 Jahre) und nur 3 Rezidive auf 69 Patienten während des 
Aufenthaltes im Asyle. Die intrauterine Behandlung hat auch auf den weiteren Verlauf der angeborenen Syphilis einen deutlich günstigen Einfluß sowohl klinisch als serologisch gezeigt.

\author{
Kasuistik. \\ Ältere Individuen. \\ Lues hereditaria tarda.
}

Fall 1. 70/1907. Inez Walborg A. 7 Jahre.

Von der Mutter wird nichts angegeben. St. pr. 18./III. 1907. Etwas imbecill. Tubera frontalia hervortretend. An den Mundwinkeln strahlenförmige Narben. Die Zähne zeigen fehlerhafte Implantation, nicht typisch Hutchins on sche. Gehörvermögen etwas herabgesetzt. An den Vorderseiten der Tibiae können einige Unebenheiten palpiert werden. An beiden Augen Keratitis parenchymatosa und an dem linken Glaukom. Die positive W. R. nach sieben $\mathrm{Hg}$-S.-Kuren schwächer und nach noch einer negativ.

Fall 2. 9/1909. Ellen Theresia H. 8 Jahre.

Die Mutter war 1895 (Nr. 419) im Krankenhause St. Göran wegen Primäraffekt an der Oberlippe antiluetisch behandelt; weitere Angaben fehlen. Die Patientin wrrde am 7./I. 1909 im Krankenhause aufgenommen; hatte doppelseitige Keratitis parenchymatosa, sonst nichts; die Seroreaktion war positiv und blieb so trotz vier Hg-S.-Kuren während zweier Jahre.

Fall 3. 75/1908. Karl Axel Wilhelm W. 10 Jahre.

Die Mutter (186/1892) im Krankenhause St. Göran wegen ansgebreiteter sekundärsyphilitischer Erscheinungen gepflegt; war damals in Grossess; das manifest hereditärluetische Kind starb 11 Wochen alt. Im April 1893 totgeborenes Kind. 1895 im Juli ein Kind, welches 1899 gestorben ist. 1897 gebar sie den Patienten und 1901 ein Mädchen, das gesund sein soll.

Der Patient soll vor der jetzigen Krankheit keine Symptome von Lues aufgewiesen haben, und hat bei der Untersuchung doppelseitige Keratitis parenchymatosa und sonst nichts; die positive W. R. wurde erst nach sieben Hg-S.-Kuren schwächer, dann abwechselnd positiv und negativ; die letzte Untersuchung (26./X. 1911) ergab negative W. R.

Fall 4. 26/1908. Anna Greta O. 12 Jabre.

Die Mutter wurde am 4./XI. 1894 (Nr. 578) wegen frischer Syphilis im Krankenhause St. Göran aufgenommen, war gravid, bekam Hg-Behandlung und gebar 6./III. 1895 die Patientin, welche multiple Osteochondritiden zeigte. Sie ersehien wieder 12 Jahre alt mit Iritis $d x$. und Keratitis parenchymatosa bil. AuBerdem zeigte sie verschiedene entstellende Deformitäten der Extremitäten, genau entsprechend den Osteochondritiden, die sie als Säugling zeigte; Röntgenphotographie zeigt einen vollständigen Schwand der Beinsubstanz an diesen Stellen; nach vier 
Klin. Beobachtungen über die Prognose der kong. Syphilis.

Hg-S.-Behandlnngen wurde die Seroreaktion negativ befunden und war auch so im Mai 1912.

Fall 5. 441/1910. Gösta Robert H. 14 Jahre.

Keine anamnestische Angaben. Jetzt Keratitis parenchymatosa dx., Dentes Hutchinsoni und Periostitides tibiarum; positive W. R. Nach 0.2 Hata (intramuskulär) vorübergehende Abschwächung der Hemmung. Nach 3 Hg-Injektionskuren fortwährend positive W. R.

F a 11 6. 38/1907. Ragnhild P. 14 Jahre.

Die Anamnese enthält nur negative Angaben. Jetzt doppelseitige parenchymatöse Keratitis, fehlerhafte Implantation der Zähne, sonst nichts. Nach $3 \mathrm{Mk}$-Kuren and $4 \mathrm{Hg}$-S.-Kuren negative Seroreaktion.

Fal1 7. 148/1912. Birger Karl F. 17 Jahre.

Die Mutter weiB nichts von einer luetischen Infektion, ist jetzt symptomfrei und hat negative W. $R$. Der Patient soll als nengeboren keine verdächtigen Lueserscheinungen gehabt haben, soll aber. kränklich gewesen sein; die Zunge erkrankte bei 4 Jahren, die Angen bei 10 . St. pr. Graziler Körperbau; allgemeine Hautfarbe graublaß; in den beiden Corneae Flecken nach ausgeheilter Keratitis parenchymatosa, Trübungen im linken Glaskörper. Ausgeprägte Sattelnase; Rhinitis atrophicans. Zähne sehr unregelmäßig mit bedeutenden Lücken zwischen einander. Die Zunge im ganzen vergrößert und verdickt, Dorsum von dichten, höckerigen, von tiefen Furchen begrenzten papillomatösen Wucherungen bedeckt; an den Seitenrändern graublauweiße, lejstenförmige Epithelverdickungen, Stomatitis. Gehör ohne Bemerkung. Genitalia wenig entwickelt (soll niemals Erektionen oder Pollutionen gehabt haben). Herz und Lungen unverändert. Leber bedeutend vergrößert; untere Grenze in der Mamillarlinie $5 \mathrm{~cm}$ unter dem Brustkorbsrande; vordere Fläche grobhöckerig zu palpieren. Kein Albumen oder Zucker im Harne. Nervensystem (Reflexen) ohne Veränderungen. Intelligenz völlig normal. W. R. - Drei Salvarsaninjektionen à 0.3 (intravenös) ohne sichtbare Wirkung.

F all 8. 271/1909. Karl Johann S. 17 Jahre.

Anamnese negativ. Sattelnase; Perforatio septi, Rhinitis. Periostitis tibiae sin., positive W. R., welche nach acht Hg-Injektionskuren sich etwas schwächer zeigte.

F a 11 9, 433/1909. Karl Bruno K. 18 Jahre.

Der Patient kann keine bestimmten anamnestischen Angaben machen. Abnebmendes Sehvermögen seit drei Jahren; seit einem Jahre Schwierigkeiten beim Gehen. St. pr.: Sattelnase und Perforation des Septum. Vollständige Amaurose; Atrophie beider nervi optici. Gehör gut, Dentes irregulares; Romberg positiv; Patellarreflexe rechts keine, links herabgesetzt; keine Parästhesien, keine lanzinierende Schmerzen. W. R. positiv. Trotz großer Mengen K. J. und Hg (acht Säckchenkuren) allmähliche Verschlimmerung des Zustandes. Nach 2 Jahren in Hemiplegia gestorben. Sektion: Hämorrhagia cerebri e. Ruptura A. basilaris.

Fall 10. 37/1911. Anna 0.28 Jahre. 
Keine Angaben von der Mutter zu erhalten. Mit sieben Jahren Augen- und Ohrenkrank; vor einem halben Jahre geisteskrank; unter antiluetischer Behandlung fast vollständig gebessert. Jetzt keine Wahnvorstellungen $\mathfrak{u}$. ä. Klagt über Sausen im Kopfe. Trübungen der Hornhaut beiderseits; Gehör herabgesetzt. Tibiae uneben. Romberg positiv. W. R. + +十.

Fall 11. 173/1891. Georg D. 22 Jahre.

Waisenkind. So lange er sich erinnern kann, hat er schlecht und nasal gesprochen. Beim ersten Aufenthalte im Krankenhause hatte er Perforation des harten und weichen Gaumens, Deformitäten der Epiglottis, chronisch - inflammatorische Veränderungen des Kehlkopfes, schlechtes Gehör und Atrophie der Testikeln. Wiederholte Hg-Behandlungen haben die Veränderungen nicht das Geringste beeinflußt; 1910 war die Seroreaktion negativ.

Fall 12. 210/1910. Sofia Charlotta J. 37 Jahre. Verheiratet.

Die Mutter soll während der Schwangersehaft an Lues gelitten haben; zwei jüngere Geschwister sollen gesund sein. 18 Jahre alt, Keratitis parenchymatosa und allmäbliche Deformierung der Nase. 29 Jahre alt, verheiratet; nach einem Jahre ein Kind, welches nach 18 Tagen zugrunde ging. Kein Abort. Ein zweites Kind, jetzt $6 \frac{1}{2}$ Jahre alt, ist symptomenfrei und gibt negative W. R. St. pr. Ausgeprägte Sattelnase, Perforation des harten Gaumens, Kornealflecke links und Auftreibungen beider Tibiae; W. R. +++ .

F all 13. Poliklinik 230/1912. Märta Sofia K. 24 Jahre. Verheiratet.

Die Mutter luetisch angesteckt während des Sommers 1887; bekam vier Hg-Injektionen. Patientin 8./IV. 1888 geboren, ausgetragen, ohne bestimmte luetischen Erscheinungen. Eine 1889 geborene Schwester starb im zweiten Lebensmonate an Bronchitis (?). Eine 1889 geborene Schwester soll immer gesund gewesen sein, ist jetzt erscheinungsfrei and gibt negative W, R.

Die Patientin selbst soll immer kränklich gewesen sein; kann keine direkte luesverdächtige Erscheinungen angeben. Defloriert (vom späteren Ehemanne) mit 17 Jahren (1905); gebar 15./III. 1906 eine tote luetische Frucht; 18./IV. 1907 luetische Fehlgeburt im siebenten Monate; 1908 wieder geschwängert; jetzt $\mathrm{Hg}$-Kur: ausgetragenes symptomenfreies Kind, das jetzt keine anderen für Syphilis verdächtigen Symptome als eine Assymmetrie des Kopfes aufweist und negative W. R. gibt. Nachher keine Hg-Behandlung. 6./VIII. 1912 neue Geburt (= Fall Nr. 99). Kongenitalluetisches Kind.

St. pr. Graziler Körperbau, Hautfarbe blaß, an den Mundwinkeln feine strahlenförmige Narben, sonst kein einziges Zeichen von Lues; kein Leukoderma, keine lokale oder allgemeine Adenitis. Zähne unregelmäßig, aber sehr wenig. Sinnesorgane, innere Organe und Nervensystem ohne nachweisbare Veränderungen. W. $R$. +++ .

Der Ehemann verneint syphilitische Infektion, hat keine Erscheinungen von Syphilis und negative $W$. $R$.

Patientin gibt bestimmt an, mit keinem anderen Manne als ihm verkehrt zu haben. 
Klin. Beobachtungen über die Prognose der kong. Syphilis. 137

\section{Neugeborene Kinder.") \\ Gruppe I.}

A.

F a I1 14.

53/1907. Mathilda A. 40 Jahre. Köchin.

Partus: 22./2. 1907.

Inf.: November 1906.

Stat. pr.: 2./3. 1907. Skierose lab. sup. $\quad 0$

+ Adenit. + Roseola.

54./1907. Erik A. $\quad$ L. H. 37

Geboren: 22./2. 1907 .

Stat. pr.: 2./3. 1907. Koryza + Psor. plantaris + Pap. muc.

Fal1 15.

299/1911. Wanja Maria J. 21 Jahre. Modistin.

Inf.: Anfang 1911.

Partus: 11./8. 1911.

Stat. pr.: 26./8. 1911. Pap. muc, vulv.

+ Adenit. univ.

26./8. 1911

300/1911. Erik Wilhelm J.

Geboren: 11./8. 1911.

Stat. pr.: 26./8. 1911. S-frei. Gew. 28./8. ++ $3900 \mathrm{~g}$.

Fall 16.

121/1911. Elsa Paulina Viktoria K. 24 Jahre. Geschäftsgehilfe.

Inf.: Ende 1909. (Wahrscheinlich.)

Partus: $\quad 18 . / 5.1910$.

St. pr.: $\quad$ 31./3. 1911. Pap. muc. ad

0 anum. + Exanth. pap.

12./4. +++ univ. + Adenit. ing. +

Pap. capillitii + Iritis dx.

122/1911. Inga Elsie K.

Geboren: $18 . / 5.1910$.

Stat. pr.: 31./3. 1911. S-frei. Gut genährt.

31./3. $++t$

1) Die nachfolgenden kurzen Krankengeschichten sind der Übersichtlichkeit wegen in drei Kolumnen aufgestellt: die erste enthält die klinischen Daten, die zweite die Behandlung und die dritte die Seroreaktion. "L. H." bedeutet, daß das Kind später ins "Lilla Hemmet" aufgenommen und unter dem betreffenden Nummer in der "Tabelle zu finden ist. 


\section{Fall 17.}

45/1903. Ingeborg J. 24 Jahre. Dienstmädchen.

Gravida: Juli 1902 .

Inf.: $\quad$ Oktober 1902.

Partus: $\quad 17 . / 2$. 1903 .

Stat. pr.: 25./2. 1903. Pap. muc. vulv.

+ Leukoderma + Roseola.

46/1903. Linnea J.

Geboren: 17./2. 1903 .

Stat. pr.: $25 . / 2.1903$. Nicht ausgetragen.

Atrophie, Gewicht $2100 \mathrm{~g}$.

F a Il 18.

110/1900. Helga Helena A. 25 Jahre. Fabriksarbeiterin.

In f.:

Frühjahr 1900 .

Partus: $\quad 2 . / 7.1900$.

Stat. pr.: 10./7. 1900. Sklerose + Adenitis + Roseola.

111/1910. Wilhelm A.

I. H. 3.

Geboren: 2./7. 1900 .

Stat. pr.: 10./7. 1900. S-frei. Gewicht $3700 \mathrm{~g}$. Koryza.

Fall 19.

238/1909. Elsa Alfrida R. 20 Jahre. Ehefrau.

Inf: : Juli 1909.

Gravida: Seit Februar 1909.

L. H, 17.

Partus: $\quad$ 17./10. 1909.

Stat. pr.: 9./12. 1909. Sklerosereste + Adenit. + Pap. muc. vulv. + Exanth. mac. pap. +
Psoriasis plant, et palm.

10./12.-6./1.

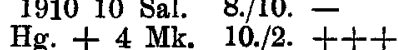
14./2.-14./3. 10 Sal. Hg. +

11./1. 1910. Leukoderma. 2. $\mathrm{Mk}$.

239/1909. Lucia Antoinette R.

Geboren: 17./10. 1909.

Stat. pr.: 9./12. S-frei.

12./2. 1910. Mors in Broncho-

$0 \quad 9.12$.

8./1. $1910-$ pneumonie.

10./2. -

Fall 20.

252/1911. Elna N. 30 Jahre. Köchin.

Inf.:

1.5. 1911.

Gravida: Seit November 1910.

0

Stat. pr.: 15./6. 1911. Skler. lab. dx. + Pap. muc. vulv. + Aden. ing. + Gravid. Mens. VIII.

16./6. Salvarsan $0.4 \mathrm{I}$

16./6. +++

Partus: $\quad 7 . / 7$.

S tat. pr.: 24./7. Sklerosereste + Papelr. 24./7. d:o. II. 23./7. +++ 
Klin. Beobachtungen über die Prognose der kong. Syphilis. 139

253/1911. Hjalmar Gustav N.

L. H. 63 .

Geboren: 7./7. 1911. Ausgetragen.

Gewicht $2750 \mathrm{~g}$.

Stat. pr.: 24./7. Atrophie 芩 Leberver- 24./7. - 8./9. Hg. größerung. 27./10. Gewicht $6500 \mathrm{~g}$.

Säckuhen à $1 / 2$ g $27 . / 7$. + +

Fall 21.

233/1911. Fanny Maria L.

Gravida: Seit Januar 1911.

Inf.: $\quad$ Frühjahr 1911.

Stat. pr.: 10./7. 1911. Sklerose + Pap. 0*4 Salv. I 11./7. muc. vulv. et tons.

Partus: $\quad 11 . / 9$.

232/1911. Otto L.

dto. II $3 . / 8$.

dto. III 28./8.

1./8. +++

dto. IV 4./10.

dto. V 30./10. 1./11. +++

dto. VI 17./2.

1912.

Geboren: 11./9. 1911.

Stat. pr.: 22./9.1911.Atrophic(Hepatitis)

Gewicht $2400 \mathrm{~g}$.

Mors 25./9. Verblutung

(Hemophili).

24. $/ 8$. +++

20./9. +++

Fall 22.

141/1902. Karolina A. 27 Jahre. Plätterin.

Gravida: Ende August 1901.

Inf.: Wahrscheinlich Nov. 1901.

Stat. pr.: 2./1. 1902. Sklerose, Oedema Hg-Säckchen dur. + Pap. trunci + Ro- à 7 g 19 Tage. seola.

Partus: $\quad 18 . / 5$, 1902.

Stat. pr.: 27./5. 1902. S-frei.

142/1902. Karl A.

L. H. :2.

Geboren: $18 . / 5.1902$.

Stat. pr.: 2./6. 1902. Psoriasis plant. + Koryza.

Gew. $3150 \mathrm{~g}$.

Fall 23.

127/1910. Natalia A. 20 Jahre. Dienstmädchen.

Inf.: Herbst 1909.

Stat. pr.: 28./12. 1909. Sklerose, 28./12. 1909 bis Oedema durum + papul. 5./2. $19107 \mathrm{Mk}$.

Partus: $\quad 29 . / 5.1910$.

Stat. pr.: 29./6. 1910. Leukoderma $\quad$ 14./6. Mk. 1./3., 15./3.,

22./9. - 
128/1910. Josef Albin A.

Geboren: 29./5. 1910. Gewicht bei der

I. H, 46 .

Geburt beinahe $3000 \mathrm{~g}$.

Stat. pr.: 29./6. Exanthema papulosum. 22./9. S-frei.

Fall 24.

102/1901. Emma E. 23 Jahre. Fabriksarbeiterin.

Inf.: $\quad$ Anfang 1901 .

Stat. pr.: 12./4. 1901. Sklerose. Roseola pap. muc. vulv. et ani.

Partus: $\quad$ 7./6. 1901.

Hg-Säckchen à 6 g 40 Tage.

103/1901. Elsa E.

L. H. 18.

Geboren: 7./6. 1901.

Stat. pr.: 14./6. 1901. S-frei.

F a 1125.

202/1908. Ellen J. 31 Jahre. Dienstmädchen.

Inf.: $\quad$ Frühjahr 1908.

Stat.pr.: 18./6. 1908. Sklerose.

Partus: $\quad$ 2./8. 1908.

Hg-Säckchen à 8 g 40 Tage +4 Sal. Hg.

203/1908. Ella J.

L. H. 40 .

Geboren: 2./8. 1908 .

Stat. pr.: 10./8. 1908. S-frei.

Fall 26.

339/1908. Therese W. 23 Jahre. Plätterin.

Inf.: Wahrscheinlich vor 2 Monat.

Gravida: Seit 5 Monaten.

Stat. pr.: 1./12. 1908. Sklerose, Roseola.

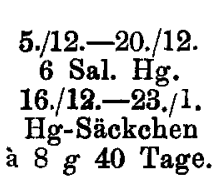

2./12. +++

6 Sal. Hg.

16./12.-23./1.

à 8 g 40 Tage.

1./3. Erysipelas faciei.

3./2. $1909-$

Partus: $\quad 2 . / 3$

6./3. Mors in Septikämie.

$68 / 1909$. Ungetauft W.

Geboren: 2./3. 1909. Ausgetragen.

$\mathrm{S}$ tat. pr.: 2./3. Gut genäbrt. Keine Zeichen von Syphilis.

Septikämie.

Mors 5./3. 
Klin. Beobachtungen über die Prognose der kong. Syphilis. 141

B.

Fall 27.

223/1908. Anna H. 26 Jahre. Arbeiterin.

Inf.: Wahrscheinlich Januar 1908.

Gravida: Gleichzeitig.

Partus: 24./8.

Stat. pr.: 1./9. Roseola, Psoriasis plant. 2./9.-9./10. 20

Mergandol

à $1 \mathrm{ccm}$

13./11, $-4 . / 12$.

9./11. -

14./1. 1909. Leukoderma

5 Mk. erschienen.

27./1.-12./3.

$\mathrm{Hg}$-Säckchen

12./5. Mors in Peritonitis.

à 8 g 40 Tage.

29./3. -

224/1908. Margareta $H$.

Geboren: 24./8. Ein Monat zu früh. 1./9.-10./10.

Stat. pr.: 1./9. S-froi (?).

$\mathrm{Hg}$-Säckchen

à $0.5 \mathrm{~g} 40$ Tage.

25./11.- - 7./1.

9./11. -

Hg-Säckchen

à 1 g 40 Tage.

15./2. $1909-$

13./2.-28./3.dto.

30./3. -

1./1. 1910. Mors in Broncho-

5./7.-13./8. dto. 26./4. pneumonie.

7./7. -

6. $/ 10$. -

Fal1 28.

Inf.:

68/1910. Tekla, A. 29 Jahre. Dienstmädchen.

Wahrscheinlich gleichzeitig mit der Konzeption.

Gravida: Seit Mitte August 1909.

Partus: $\quad 28 . / 3.1910$.

St at. pr.: $8 . / 4$. Pap. muc. vulv. + mac. pigment. post. pap. muc. vulv.

9./4. +++

8./4.-19./5.

Hg-Säckchen

69/1910. Ellen A.

à 8 g 40 Tage.

Geboren: 28./3. 1910. Ausgetragen.

Gewicht $2950 \mathrm{~g}$.

Stat. pr.: $\quad 8 . / 4$. S-frei.

23./4. Mors in Erysipelas.

14./4. Hg-Säck-

9./4. +++ 
Fall 29.

420/1911. Hilma V. S. 23 Jahre. Geschäftsgeh.

Inf.:

Wahrscheinlich vor einem

Jahre.

Gravida: Gleichzeitig.

Partus: Vor 5 Monaten.

Stat. pr.: 10./11. 1911. Pap. muc. vulv. 13./11. Salvarşan + Adenit. bilat. ing. et $0.4 \mathrm{I}$. mastoid. + Alopec. specif. 5./12. dto. II. 18./12. dto. III.

15./1.1912dto.IV. 27./12. +++

2./2. S-frei. 22./1. +++

421/1911. Folke Helmer S. 5 Monate.

Geboren: Ein Monat zu früh.

Ausschlag seit 7 Wochen.

Stat. pr.: $\quad$ 10./11. Exanth. mac. pap. univ. 20./11.-30./12. + Pap. muc. oris + La- Hg-Säckchen ryngit. Gewicht $4225 \mathrm{~g}$. à 1 g 40 Tage + Hydr. c. creta

10./1. Varicellae. $0.05 \times 3$.

24./1. Morbilli.

1./2. Mors.

Fall 30.

150/1900. Ada Juliana K. 27 Jahre. Gärtnerin.

Inf.: Wahrscheinlich Ende 1899.

Partus: $\quad 8 . / 9.1900$.

Stat, pr.: 16./9. 1900. Pap. muc. vulv.

151/1900. Karl Oskar K. $\quad$ L. H. 6.

Geboren: $8 . / 9$. 1900 .

Stat. pr.: 16./9. 1900. Ikterus. Hepatitis.

Fall 31.

277/1909. Ebba Charlotta H. 18 Jahre. Dienstmädchen.

In f.: $\quad$ Wahrscheinlich Frühjahr1909.

Partus: $\quad 2 . / 11.1909$.

Stat. pr.: 2./2. 1910. Großfeckige Ro- 4./2-3./3. 1910. 3./2. +++ seola + Pap. corporis + 10 Sal. Hg. + Erosiones tonsill. +undeut- $4 \mathrm{Mk}$. liches Leukoderma.

14./4. Deutliches Leukoderma.

278/1909. Sven Gustav H.

Geboren: 2./11. 1909.

Stat. pr.: 18./11. Schnupfen, ũbrigens S.frei.

L. H. 44. 
Fall 32.

Agnes Cecilia E. 17 Jahre.

Inf: : Ende 1910 .

Partus: $17 . / 8$. 1911.

Stat. pr.: 26./8. 1911. Exanth. papulos.

+ Pap. muc. vulv.

Eugen E.

L. H. 65 .

Geboren: 17./8. 1911.

Stat. pr.: 26./8. 1911. Koryza + Exanthema papul.

26./8. 1911

$+++$

Fall 33 .

236/1908. Alma J. 24 Jahre. Dienstmädchen.

In f.: Wabrscheinlich vor 1 Jahr.

Gravida: Gleichzeitig.

Partus: Anfang Dezember 1907.

Stat. pr.: 21./2. 1908. Pap. vulv. et ani 22./2.-4./4. 1910

+ Leukoderma. Sal. Hg. $+4 \mathrm{Mk}$.

5./5.-30./5. $6 \mathrm{Mk}$.

Während des

8./9. S-frei.

August $7 \mathrm{Mk}$.

9./9. -

237/1908. Lars Gunnar J. 21/2 Monate.

14 Tage nach der Geburt Ausschlag an den Nates und schwerer Schnupfen.

Stat. pr.: 21./2. 1908. Großfleckiges 21./2.-31./3. Roseola + Psoriasis plant. $\mathrm{Hg}$-Säckchen + Pseudoparalysis. à 1 g 40 Tage. 4. $/ 5 .-12 . / 6$. dto.

Während des

August $30 \mathrm{Hg}$ -

8./9. S-frei.

Säckchen.

30./9.-9./11.

9./9. +++

Hg-Säckchen

à 1 g 40 Tage. $9 . / 11$. -

Fall 34.

253/1904. Hilma Sophie O. 23 Jahre. Dienstmädchen.

Inf.:

Frühjahr 1904.

Gravida: Gleichzeitig.

Stat. pr.: 24./11. 1904. Pap. muc. valv. Hg-Säckchen

Partus: $\quad$ t Adenitis + Leukoderma. à $8 \mathrm{~g} 20$ Tage.

10/1905. Ruth 0 .

L. H. 27.

Geboren: 5./1.1905. Ein Monat zu früh.

S t at. pr.: 14./1. S-frei. Gewicht $2000 \mathrm{~g}$. 
Fall 35.

250/1899. Hulda Maria W. 20 Jahre. Dienstmädchen.

Inf.: $\quad$ Anfang 1899.

Gravida: Gleichzeitig.

Stat. pr.: 24./7. 1899. Sklerosereste + Hg-Säckchen Pap. muc. vulv. + Pap. à 5 g. Merkuriol

Partus: $28 . / 10.1899$

40 Tage.

S tat. pr.: 12./11. 1899. Angina papulosa.

251/1899. Wera W.

L. H. I.

Geboren: $28 . / 10.1899$.

Stat. pr.: 12./11. 1899. S-frei. Gewicht $2650 \mathrm{~g}$.

Fall 36.

126/1906. Anna Sophie H. 26 Jahre. Dienstmädchen.

Inf.: Ende 1905.

Gravida: Gleichzeitig.

Stat. pr.: 24./1. 1906. Sklerose + Pa- Hg-Säckchen pulae + Roseola.

Partus: 26./5. 1906. $30+13$ Tage.

125/1906. Arnold H.

Geboren: 26./5. 1906. S-frei.

L. H. 31 .

Fall 37.

194/1904. Edla S. 28 Jahre. Dienstmädchen.

Inf.: Herist 1903 .

Gravida: Gleichzeitig.

Stat."pr.: 16./5. 1904. Pap. muc. vulv. Hg-Säckchen ani et tons + Adenitis + à $8 \mathrm{~g} 40+40$ Erythema papul. + Psor. Tage.

Partus: $\quad 13 . / 9.1904$. plant. + Leukoderma.

Stat. pr.: $20 . / 9$. 1904. Leukoderma.

195/1904. Marta S.

L. H. 25 .

Geboren: 13./9. 1904 .

Stat. pr.: 20./9. 1904. S-frei.

\section{Fall 38.}

251/1908. Eva Ö. 21 Jahre. Näherin.

Inf.:

Mitte Dezember 1907.

Gravida: Gleichzeitig.

Stat. pr.: 2./5. 1908. Großfleckige Ro- 4./5.-30./5. 10 seola + Pap. muc. vulv. et Sal. Hg. +4 Mk. ani + Psoriasis plant. + 29./6.-8./8. Eros. tons. +.Leukoderma Hg-Säckehen

$\begin{array}{lll}\text { Partus: } & \text { 8./9. Graviditas mens. V. } 1908 \text { à } 8 \text { g } 40 \text { Tage. }\end{array}$

Stat. pr.: 17./9. Leukoderma übrigens Hg-Säckchen 31./10. S-frei.

à $8 \mathrm{~g} 40$ Tage. 8./12.-7./1.1909 
252/1908. Valdemar ö.

Geboren: $8 . / 9$. 1908 .

Stat. pr.: $17 . / 9$. S-frei.

Fall 39.

21./9.-1./11.

Hg-Säckchen

à $0.5 \mathrm{~g} 40$ Tage. 31./10. -

7./12.-16./1. dto.

4./2. 1909

233/1907. Elvira A. 22 Jahre. Näherin.

In f.:

Anfang 1907.

Gravida: Gleichzeitig.

Stat.pr.: 11./2.1907. Sklerose+Oedema 2 Inj.-Kuren + durum + Adenitis + Ro- 40 Hg-Säckchen

Partus: 13./9. 1.907.

234|1907. Bertil A.

Geboren: 13./9. 1907.

Stat. pr.: 27./9. S-frei.

Mors: $18 . / 2.1909$ in Bronehitis.

Fall 40.

113/1912. Beda Wilhelmina S. 23 Jabre. Dienstmädchen.

In f.:

September 1911 .

Gravida: Gleichzeitig.

Stat. pr.: 15./12. 1911. Pap. muc. vulv. V. Salvarsan

+ Adenitis inguinalis $+\mathrm{à} 0 \cdot 418 . / 12,5 . / 1$, Leukoderma. $\quad 20 . / 1 ., 9 . / 3 ., 4 . / 4$.

Partus: $\quad$ 6./5. 1912

$4 \mathrm{Mk} .16 . / 4 .-2 / 5$.

15. $12 .+++$

Stat. pr.: 14./5. Leukoderma colli.

$$
\begin{array}{ll}
22 . / 5 .-1 . / 6.3 \mathrm{Mk} . & 14 . / 5 .+ \\
& 30 . / 5 .-
\end{array}
$$

114/1912. Erik Birger S.

Geboren: 6./5. 1912.

Stat. pr.: 14./5. S-frei.

Gewicht $2700 \mathrm{~g}$.

$$
\begin{array}{ll}
\text { 14. } / 5 .-23 . / 6 . & 14 . / 5 .- \\
\begin{array}{c}
\text { Hg-Säckchen } \\
\text { à } 1 / 2 g .
\end{array} & 30 . / 5 .-
\end{array}
$$

Fall 41 .

417/1911. Anna Ottilia M. 22 Jahre. Arbeiterin.

Inf.: Anfang 1911.

Gravida: Seit Ende Januar 1911.

\section{0}

Stat. pr.: 28./8. 1911. Pap. muc. vulv. 30./8. Salv. 0.4 I 30./8. +++ + Polyadenit. + großfleckige Roseola + Graviditas Mens. 29./9. dto. II VII,

Partus: $\quad 29.110$

13./10. +++

Stat. pr.: 9./11. S-frei.

418/1911. Talis M.

Geboren: 29./10. 1911. Ausgetragen.

L. H. 70 .

S-frei. Gewicht $3220 \mathrm{~g}$.

$17 . / 3$. Gewicht $5650 \mathrm{~g}$.

Arch. f. Dermat. a. Syph. Bd. OXVI. 
Fall 42.

In f.:

82/1911. Alfrida Kristina A. 23 Jahre. Kellnerin.

Etwa 1.6. 1910

Gravida: Gleichzeitig.

Stat. pr.:

6./12. 1910 .

+ Exanth. maculo-papul H.j12.-16./1.

Partus: 25./2. 1911 .

83/1911. Margareta Alfrida A.

à 8 g 40 Tage. 16./3. $-10 . / 4$.

7./12. +++
10./3. $t++$ $6 \mathrm{Mk}$.

Geboren: 25./2. 1911. Ausgetragen.

Stat. pr.: 6./3. S-frei. Gewicht $3100 \mathrm{~g}$.

7./4. Mors in Pneumonie. Säckchen 1 ./3. 1 g-

10./3. +++

Fall 43 .

114/1908. Regina Olivia W. 34 Jahre. Waschfrau.

In $f_{n}:$

Angust 1907.

Gravida: Gleichzeitig.

Stat. pr.: 9./3. 1908. Sklerose + Roseola

Partus: 28./4. 1908 .

Hg-Säckchen

à 8 g 40 Tage

+4 Sal. Hg.

9./11. $1900-$

115/1908. Gösta W.

Geboren: 28./4. 1908. 5 Wochen zu

L. H. 41. früh.

St at. pr.: 5./5.1908. Atrophie. Gewicht $2050 \mathrm{~g}$.

Fall 44.

1/1912. Lilly Helena S. 10 Jahre.

Die Mutter infiziert 1901, gleichzeitig mit ihrer Schwangerschaft, während welcher sie mit $\mathrm{Hg}$ behandelt wurde. Pat. bei der Ge burt Pap. ad anum. Hg. Säckchen-Kur.

Stat. pr.: 2./1. 1912. Dentes Hutchinsonii.

Fall 45.

87/1912. Emma Theresia E. 25 Jahre. Telephonistin.

Inf.:

Wahrscheinlich seit Nov. 1910.

Gravida: Gleichzeitig.

Stat. pr.:
17./6. 1911. Pap. muc. vulv. 19.6. Salv. 0.4 I
+ Adenit. ing. bil.
$11 . / 7$. dto. II $\begin{array}{lll}+ & 11 . / 7 . & \text { dto. II } \\ & 3 . / 8 . & \text { dto. III }\end{array}$

Partus: $\quad 19 / 8$.

29./8, S-frei.

306/1911. Göta E.

Geboren: 19./8. Ausgetragen. Gewicht

$\begin{array}{ll} & 4300 g . \\ \text { Stat. pr.: } & 29 . / 8 \text {. S-frei. }\end{array}$

30./8. + 
Fall 46.

70/1912. Lucia Sofia L. 38 Jahre. Dienstmädchen.

Inf.:

Juni 1911

Gravida: Gleichzeitig.

Stat. pr.: 17./11. 1911. Pap. muc. valv. 21/11. 1911 et ani, linguae, Psoriasis 0.4 Salvarsan I plantaris, Polyadenitis. $\quad 6 . / 12$ d d

12./2. dto. IV

Partus: $\quad 11 . / 3$.

Stat. pr.: 18./3. S-frei.

28./3. Neurorezidiv.

2./3. -

18./3. ++

(Neuritis accustica).

4./4.-19./5.

4 Sal.Hg. $+7 \mathrm{Mk}$.

$30 . / 5$. -

71/1912. Knut Oskar Wilhelm L.

Geboren: $11 . / 3$.

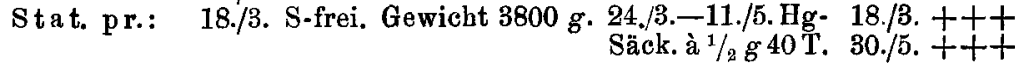

Fall 47.

63/1908. Ada Juliana F. 30 Jahre. Näherin.

In $f_{*}$ :

Frühjahr 1907 .

Gravida: Mai 1907.

Im Herbst Ausschlag im Nov.-Dez. Unterleibe, an Kopf, Brust $8 \mathrm{Hg}$-Inj.

Partus: $\quad$ 13./3. 1908.

Stat. pr.: 13./3. 1908. Lenkoderma. $\quad$ 15./3. $-14 . / 4$.

64/1908. Aino Matti F.

Geboren: 13./2. 1908 .

Stat. pr.: 13./3. 1908. Roseola + Pap. 14./3.-23./4. faciei.

Hg-Säckchen

à $1 \mathrm{~g} 40$ Tage.

29./5.-7./7. dto.

28./10. 1908. S-frei.

Fall 48.

308/1911. Elisabeth Sofia G. 18 Jahre. Kellnerin.

In f.: $\quad$ Dezember 1910.

Gravida: Seit Januar 1911.

$3 \mathrm{Hg} \cdot$ Injektionen $\quad 10 . / 6$, -

Stat +17 Einreib.

26./10. +++

Stat. pr.: 3./8. Leuk.

Partus: 23./8.

Stat. pr.: 31./8. Leukoderma + Adenit.

4./8. -

5./8. Salv. 0.4 I

309/1911. Runo Theodor G.

Geboren: 22./8. Zwei Monate zu früh.

Gewicht $1900 \mathrm{~g}$.

Stat. pr.: 31./8. 1911 Atrophie. Ikterus. LebervergröBerung.

Mors 20./9. 1911 . 


\section{Gruppe II.}

A.

Fall 49.

75/1912. Agnes Margareta N. 23 Jahre. Dienstmädehen.

Inf.: $\quad$ Im Herbst 1909.

St at. pr.: $29 / 7.1910$. Exanth. pap. univ. 30./7.-14./9. 15

+ Iritis sin. + Eros. tons. Hektin à 0.20

Gravida: Seit September 1911 (?).

Stat. pr.: 28./12. 1911. S-frei.

Partus: $\quad 31 . / 3$.

$$
\begin{array}{lll}
\multicolumn{1}{c}{\begin{array}{l}
14 . / 9 .+ \\
\text { 10./1. Salv. } 0.4 \text { I I. }+++
\end{array}} & \\
\text { 24./1. dto. II } & \\
& & \text { 10./4. }+++
\end{array}
$$

76/1912. Karl Axel N.

Geboren: 31./3. 1912. Ein Monat zu früh. Gewicht $2250 \mathrm{~g}$.

Stat. pr.: 9./4. Atrophie.

19./4. Mors in Bronchopneumonie.

Fall 50.

285/1911. Sigrid Valborg 0. 21 Jahre. Kellnerin.

In f.:

Frühjahr 1910 .

Stat. pr.: 19./8. 1910. Roseola + Pso- 20./8.-17./9. 15 riasis plant.+Pap.muc.vulv. Helstin à $0 \cdot 10$

Partus: $\quad$ 7./8. 1911.

Stat.pr.: 17./8. S-frei.

1911 im Jan. und

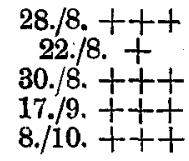

März 2 Mk.

17./8. Salv. $0.4 \mathrm{I}$

17./8. +++

6./10. Salv. 0.4 II

286/1911. Mary Valborg 0 .

Geboren: 7/8. 1911. Ein Monat zu früh.

Stat. pr.: 17./8. Exanth. pap. univ. + 17./8. Salv. $0.0117 . / 8 .+++$ Pap. muc. oris. + Hepatitis + Splenitis + Osteochondritides.

Temperatur $35^{\circ}$.

18./8. Mors.

Fall 51.

105/1901. Johanna B. 22 Jahre. Dienstmädchen.

In f.: Mitte 1900 .

S tat. pr.: 18./12. 1900. Pap. muc. vulv. Hg-Säckehen + Exanth. mac. pap. univ. à 6 g $40+40$

Partus: $\quad 14 . / 6.1901$.

Tage. 
Klin. Beobachtungen über die Prognose der kong. Syphilis. 149

106/1901. Sven B.

L. H. 9.

Geboren: $14 . / 6$. 1901 .

Stat. pr.: 22./6. S-frei. Gewicht $3180 \mathrm{~g}$.

Fall 52.

191/1905. Anna Maria R. 26 Jahre. Fabriksarbeiterin.

Inf.: $\quad$ Mai 1904.

Partus: $\quad 15 . / 8.1905$.

Drei Schmier. kuren.

Stat. pr.: 6./10. 1905. Leukoderma.

190/1905. Greta R.

Geboren: $15 . / 8$. 1905 .

Stat. pr.: 6./10. 1905. S-frei.

Fall 53.

Elin Martina B. 24 Jahre. Geschäftsgehilfe.

Inf.: $\quad$ Anfang 1905 .

Gravida: Anfang 1906.

Stat. pr.: 23./6. 1906. Roseola annulata.

Hg-Säckchen à 8 g 45 Tage.

Partus: $\quad$ 5./9. 1906. dto. 40 Tage.

Ella B.

L. H. 34.

Geboren: 5./9. 1906.

Stat. pr.: 13./9. 1906. S-frei.

Fall 54.

261/1909. Anna Josefina E.

Inf.: $\quad$ Mai 1908.

Stat. pr.: $\quad 20 . / 8.1908$. Sklerose, Oedema 22./8.-19./9. 10 durum, Adenitis, Roseola, Sal. Hg. $+4 \mathrm{Mk}$. Pap. muc. + Eros. tons. + Pap. muc. vulv.

Gravida: Im Januar 1909.

Stat. pr.: $16 . / 6$ 1909. Leukoderma.

Im März 2 Mk.

16./6. $-26 . / 7 . \mathrm{Hg}-18 . / 6 .+++$

Säckchen à $8 \mathrm{~g}$

40. Tage.

Partus: 25./10. 1909.

10./9.-21./10.dt. 11./9. +++

262/1909. Alf Uno E.

Geboren: 25./10, 1909. Ausgetragen.

Stat. pr.: 2./11. 1909. S-frei.

L. H. 47.

Fall 55.

8./11. -

Mathilda 0. 25 Jahre. Dienstmädchen.

In f.: $\quad$ Ende 1910.

Stat. pr.: 23./2. 1911. Exanth. mac. pap. 3 Inj.-Kuren. + Pap. muc. vulv. + Adenit.

Sten 0 .

Geboren: 15./11. 1911 .

Stat. pr.: 21./2. 1912. S-frei. 
Fall 56.

Fanny K. 23 Jahre. Dienstmädchen.

Inf.: $\quad$ Vor ungefähr einem Jahre.

Stat. pr.: 23./1. 1908. Pap. mac. ani +4 Injektionen + pap. Syphilid.

Partus: $\quad 20 . / 3.1908$.

Margit K.

Geboren: 20./3. 1908 .

Stat. pr.: 26./3. 1908. Atrophie.

Mors 30./7. 1908 in Gastro enteritis. Sektion: keine Zeichen von Syphilis.

Fal1 57.

Inf.:

80/1903. Carolina Sofia N. 32 Jahre. Fabriksarbeiterin.

24./2. 1902. Pap. muc. vulv. Hg-Säckchen et ani + Leukoderma. à $8 g 40$ Tage. dto. 15 Tage.

Gravida: August 1902, $100 \mathrm{Hg}-$ Pillen.

Partus: 23./3. 1903.

Stat.pr.: 31./3. 1903. Leukoderma.

81/1903. Gustav N.

L. H. 19 .

Geboren: 23./3. 1903 .

Stat. pr.: 31./3. 1903. S-frei. Gewicht Hg-Säckchen $2350 \mathrm{~g}$.

Stat. pr.: 22./8. Exanth. pap. + Koryza.

Fall 58.

Hilma Constantia G.

Inf.: $\quad$ Vor $1 \frac{1}{2}$ Jahren.

3 Schmierkuren.

89/1905. Dagmar G.

L. H. 28.

Geboren: 16.4 . 1905 .

St a t. pr.: 27./5. 1905. Koryza + Exanth. mac. pap. + Pap. muc. vulv. et oris. Gewicht $2925 \mathrm{~g}$.

Fall 59.

Julia Idalia Ch. 25 Jahre. Dienstmädchen.

Inf.: Wahrscheinlich Anfang 1898.

Partus: $\quad 31 . / 5.1899$.

Stat. pr.: 7./9. 1899. Psoriasis plant. 1 Schmierkur. Leukoderma.

Erik Ch.

L. H. 2.

Geboren: $31 . / 5$. 1899 .

Stat. pr.: 7./9. 1899. Atrophie, Impetigo cont. 
Fall 60 .

190/1908. Arvida K. 25 Jahre. Dienstmädchen.

In f.: $\quad$ Wahrscheinlich im Herbst

$$
1906 .
$$

Gravida: Von Mai 1907.

Partus: 5. Januar 1908.

Stat.pr.: 11./2.1908. Pap. muc. vulv. + 19./2.-17./3. 10

Leukoderma + Psoriasis Sal. Hg. $+4 \mathrm{Mk}$. palm. et plant. + Iritis. 25./5.-25./4.7Mk.

191/1908. Oscar Fabian K.

Geboren: 5./1. 1908. Ausgetragen. 3 Wrichen nach der Geburt Ausschlag.

Stat. pr.: 11./2. 1908. Exanth. mac. pap. univ.

\section{B.}

Fall 61.

Emma Wilhelmina L. 27 Jahre. Kellnerin.

Inf.: $\quad$ Herbst $1899 . \quad 0$

Stat. pr.: 1899. Sklerose. Ausschlag.

Partus: 15./2. 1902.
Harriet L.
L. H. 16 .

Geboren: 15./2. 1902 .

Stat. pr.: 13./5. 1902. Exanth. pap. + Koryza.

Fall 62 .

290/1911. Olga Maria K. 21 Jahre. Kellnerin.

Inf.: $\quad$ Im Herbst 1908 . Febr.-April 1909

$2 \mathrm{Hg}$-Inj.-Kuren.

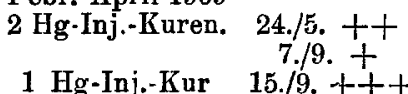

$1 \mathrm{Hg}-\mathrm{Inj}$.-Kur

Gravida: Seit März 1910.

Partus: 11.12. 1910.

Stat. pr.: 10./3. 1911. Leukoderma.

28./10. -

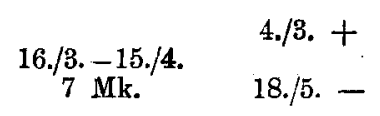

90/1910. Sven Viktor Emanuel K.

Geboren: 11./12. 1911. 3 Wochen zu früh. Gewicht $3600 \mathrm{~g}$.

Stat. pr.: 10./3. 1911. Atrophie + Koryza.

L. H. 58 .

Fall 63 .

Stina P. 29 Jahre.

Inf.: $\quad$ Anfang 1909 .

Partus: 28.7. 1911.

5 Inj.-Kuren.

Stat. pr.: 21./8. 1911. S-frei.

11./3. +++ 
Sonja P.

Geboren: $28 . / 7$. 1911.

Stat. pr.: 21./8. 1911. S-frei.

Fall 64 .

429/1911. Signe F. 27 Jahre. Köchin.

Inf.: $\quad$ Wahrscheinlich 1909.

Stat. pr.: 28./9. 1910. Pap. lichenoid. et aggregat. auf den Beinen.
L. H. 64.

21./8. 1911

Partus: Anfang September 1911.

Stat.pr.: 14./11. 1911. S-frei.

0

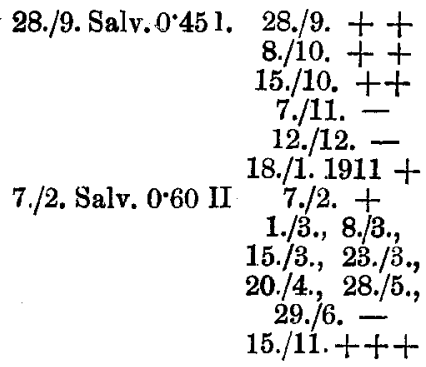

430/1911. Roland F. 2 Monate.

Stat.pr.: Koryza, Psoriasis plant. et palmaris.

Gewicht 17./11. $4800 \mathrm{~g}$.

16./11. Hg-Säck-

11/1912. Mors in Bronchopneumonie. chen à 1 g17Tage. 15./11. +++

Sektion: Hepatitis interstitialis luetica.

Fall 65 .

131/1908. Eleonora A. 37 Jahre. Näherin.

Iuf. : 1906.

Partus: $\quad$ 21./5. 1908.

6 Inj.-Kuren.

Stat. pr.: 27./5, 1908. S.frei.

6./6.-15./7. Hg-

Säck. à $8 g 40 \mathrm{~T}$.

10. 10 . -

132/1908. Ebba Karola A.

Geboren: 21./5. 1908.

Stat. pr.: 27./5. S-frei.

Fa11 66.

6./6. - 17./7. $\mathrm{Hg}-$

Säck. à $0 \cdot 5 \mathrm{~g} 40 \mathrm{~T}$.

151/1907. Jenny S. 27 Jahre. Näherin.

Inf.: $\quad$ Mitte 1905.

Partus: 1./4. 1907.

Hg-Säckchen à $8 g$
40 Tage + 11 Injek-

tionen während der Grossesse.

Stat. pr.: 19./7. 1907. S-frei.

152/1907, Harriet S.

L. H. 35 .

Geboren: 1./4. 1907.

Stat. pr.: 19./7. 1907. Koryza. 
Fall 67.

138/1909. Anna Ö. 30 Jahre. Ehefrau.

Inf.: $\quad 1907$.

Stat. pr.: 15./8. 1907. Pap. muc. vulv. 15./8.-18./9. 6 + Exanth. papulat. Atoxyl. à $0.40+$ 6 Sal. Hg. 2 Inj.-

Kuren à $7 \mathrm{Mk}$.

1 Inj.-Kurà $7 \mathrm{Mk}$.

Partus: $\quad$ 18./3. 1909 . + einige Inj.

Stat. pr.: $\quad 3 . / 6$. S-frei.

4./6.-23./6. Hg-

Säckchen à $8 g$

19 Tage. 21./9., 26./9. 2 Mk.

139/1909. Edvard ö.

5./6. -

4. $10 .+++$

Geboren: 18./3. 1909. Fine Woche nach der Geburt Schnupfen.

30./5. Ausschlag.

Stat.pr.: 3./6. 1909. Exanthema mac.papulos. univ. + Koryza + Rhagad ani.

\section{Hg-Säckchen à $\mathbf{j}$ g 19 Tage. \\ Mehrere Hg-Kuren.

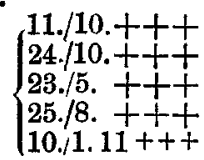

Fall 68.

81/1909. Eva Maria K. 25 Jahre. Kellnerin.

Inf.:

1907.

84Mergalkapseln +10 Sal. Hg. + $4 \mathrm{Mk}$. $+\mathrm{Hg}$-Säck -

Partus: $\quad 9 . / 3.1909$. chen à $8 \mathrm{~g} 28 \mathrm{~T}$.

Stat.pr.: 16./3. 1909. Leukoderma.

82/1909. Gustav K.

L. H. 43 .

Geboren: $9 . / 3.1909$

Stat.pr.: 16./3. Koryza.

Fall 69.

Ellen J. 40 Jahre.

Inf.: $\quad$ Ende 1908.

Stat. pr.: April 1910. Papul. aggregat.

3 Inj.-Kuren.

Partus: $\quad$ 21./11. 1910.

?/4. 1910 .

$+++$

L. H. 51 .

Geboren: 21./11. 1910

S-frei. 
Fall 70.

14/1910. Maria K. 30 Jahre. Dienstmädchen.

In f.: $\quad 1907$.

7 Inj.-Kuren, die letzte 7./10.1909.

Partus: $\quad 29 . / 11.1909$.

Stat. pr.: 15./1. 1910. Leukoderma + Narben nach Pap. mue. vulv. 24./1.-24./2.1910

15. $1 .+++$

10 Asurol +4

Huile grise.

15/1910. Gösta K.

11./4.-12./5.7Mk.

1./3. ++

6./7.-5./8. $7 \mathrm{Mk}$ 。

$9 . / 6$. -

1./10.-1./11. $7 \mathrm{Mk}$.

15./8. -

Geboren: 29./11. 1909. Ausgetragen.

Gewicht $2780 \mathrm{~g}$.

Stat. pr.: 15./1. 1910. Exanth. pap. + 15./1.-25./2.Hg- 15./1. +++ Koryza + Rhagad oris.

Säckchen à $1 / 2 g$ 40 Tage.

11./4. - 20./5. Hg-

Säckchen à $1 \mathrm{~g}$ 40 Tage.

6./7.-14./8. dto. 16./8. Salvarsan 0.03 (intram.)

5./9. - 14./10.Hg -

Säckchen à $1 \mathrm{~g}$ 40 Tage.

9. $4 .+++$
9./6. +++

15./8. + $30 . / 8+$

8./9. + 23./9. +++ 6./10. ++ 29./10. 2.11. + $26 / 1.11+++$

Fall 71.

191/1909. Ida L. 21 Jahre. Dienstmädchen.

In f.: Januar 1907.

Gravida:

Gleichzeitig.

Partus: $\quad 3 . / 8$. Die Frucht während der Entbindung gestorben; bei der Obduktion keine luetischen Symptome.

Stat. pr.: $\quad$ 10./8. Pap. muc.vulv. +Leuko- $11 . / 8$ - $4 . / 9.9$ derma.

Atoxyl + 6./9.-

Partus: $\quad$ 21./6. 1909.

20./9. $6 \mathrm{Sal}$. Hg.

Stat. pr.: $\quad 20 . / 7$. 1909. Leukoderma.

29./7.-25./8. 10 Sal. Hg. $+4 \mathrm{Mk}$.

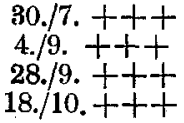


Klin. Beobachtungen über die Prognose der kong. Syphilis. 155

$$
\begin{aligned}
& 20 . / 10 .-19 . / 11.7 / k \text {. } \\
& \text { 21./4. S-frei. } \\
& \text { 3./1.10-7./5. 7Mk. } \\
& \text { 27./4.-27./5.7 Mk. } \\
& 4 . / 1.1910++ \\
& \text { 24./4. }++ \\
& \text { 6./6. - } \\
& \text { 14./10. - }
\end{aligned}
$$

191/1909. Oskar Ferdinand L.

Geboren: 21./6. 1909.

S tat. pr.: 28./7. 1909. Exanth. pap. univ. $28 / 7 .-7 . / 9$. Hg- 30./7. +++ + Rhagad oris + Koryza Säckchen à $0.5 \mathrm{~g}$ + Hepatitis.

$$
40 \text { Tage. }
$$

20./10.-28./11. dt

19./10. +++ $4 . / 1.10+++$

8./1.-17./2. dto à 1 g 40 Tage.

27./4.-5./6. dto.

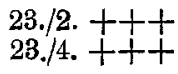
à $1.5 \mathrm{~g} 40$ Tage.

$$
\begin{gathered}
\text { 6./6. }+++ \\
\text { 9./9. }+ \\
\text { 14./10. }+++
\end{gathered}
$$

Fall 72.

52/1902. Elin Maria J. 23 Jahre. Dienstmädchen.

Inf.: Anfang 1900.

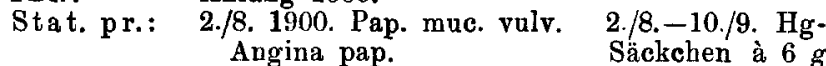
Angina pap. 40 Tage

Stat. pr.: 5./3. 1901. Pap. muc. ani. dto.à 8 g 40Tage.

Gravida: Mai 1901 .

Stat. pr.: 25./11. 1901. Leukoderma + Pap. muc. vulv.

Partus: $\quad$ 4./2. 1902.

St at. pr.: 1./3. Leukoderma.

53/1902. Ernst J.

Geboren: 4./2. 1902. Gewicht $3470 \mathrm{~g}$.

Stat. pr.: $1 . / 3$. S-frei. Gewicht $2710 \mathrm{~g}$.

Hg-Säckchen à $6 \mathrm{~g} 37$ Tage.

L. H. 11.

\section{Fall 73 .}

25/1905. Emma Charlotta M. 22 Jahre. Dienstmädchen. Inf.: Ende 1902.

Stat. pr.: 11./4. 1903. Pap. muc. vulv. + Roseola + Leukoderma. Hg-Säckchen à8g

Partus: $\quad$ 17./1. 1905. $40+40+40$ Tage.

Stat. pr.: 26./1. 1905. Leukoderma + Pap. muc. vulv.

26/1905. Nils $M$.

Geboren: 17./1. 1905.

Stat.pr.: 26.1. 1905. S-frei. Gewicht $3700 \mathrm{~g}$.

L. H. 26. 
Fall 74.

116/1909. Anna Theresia A. 19 Jahre. Dienstmädehen.

Inf.: $\quad$ Dezember 1906.

3 Schmierkuren +4 Inj.-Kuren, das letzte Mal

Gravida: Seit Juli 1908. am 11./1. 1906.

Stat. pr.: 25./1. 1909. Leukoderma. 27./1.-7./3. HgPartus: $\quad$ 21./4. 1909. Säckchen à $8 \mathrm{~g}$ 40 Tage.

15./5.-23./6.dto. 13./2. 19./3. 2./8.-5./9. dto.

117/1909. Augnst Gustav A. Geboren: 21./4. 1909. S tat. pr.: 30.4 . Gewicht $4420 \mathrm{~g}$. S-frei.

15./5. $-23 . / 6$.

Fall 75.

402/1911. Vendela Viktoria L. 27 Jahre. Kellnerin.

Inf: : Im Herbst 1909.

Partus: 20./4. 1910. 3 Monate zu früh.

Fötus totgeboren.

Stat. pr.: 29./4. 1910. Leukoderma. $\quad 29 . / 4 .-8 . / 6$.

Gravida: Januar 1911.

Partus: $15 . / 10.1911$.

St at. pr.: $30 . / 10$. 1911. Leukoderma.

Hg-Säckchen à 8 g 40 Tage. 4 Inj.-Kuren, die letzte Juli 1911.

1./11. -

403/1911. Elsa Viktoria Viola L.

Geboren: 15/10.1911. Ausgetragen.

L. H. 69 .

Stat. pr.: $\quad 30 . / 10$. S-frei.

C.

Fa 1176.

225/1906. Gerda Viktoria L. 22 Jahre. Näherın.

Inf.: Vor 3 Jahren.

Partus: $\quad$ 17./9. 1906.

6 Schmierkuren.

Stat. pr.: 25./9. 1906. S-frei.

226/1906. Seth L.

L. H. 32.

Geboren: 17./9. 1906.

Stat. pr.: 25./9. 1906. Koryza + Hepatitis. 


\section{Fall 77.}

283/1909. Elvira Mathilda A. 24 Jahre. Näherin.

In f.:

Dezember 1906.

Gravida: Gleichzeitig.

Stat. pr.: 11./2.1907. Sklerose+Oedema 12./2.-14./3. 10 durum + Adenit. + Ero- Sal. Hg. + $6 \mathrm{Mk}$. siones + Roseola. 12./4.-13./5.7 Mk.

Partus I: 19./9. 1907. S-frei. Gut ge- 28./6.-6./8. nährt (L. H.).

Gravida: Dezember 1908.

Partus II: 6./10. 1909 .

Stat. pr.: 28./11. 1909. S-frei.

284/1909. Stig William A.

$\mathrm{Hg}$-Säckchen

à $8 \mathrm{~g} 40$ Tage.

16./10.-17./11.

$$
7 \mathrm{Mk} \text {. }
$$

23./12.-3./2.

$19087 \mathrm{Mk}$.

Jan. 1909.7 Nk.

Geboren: 6./10. 1909. Ausgetragen.

Gewicht $4500 \mathrm{~g}$.

Stat. pr.: 23./11. 1909. Exanth. pap.

$$
\begin{aligned}
& \text { 24./11. } \\
& 7 \text { Mk. }
\end{aligned}
$$

13. $1.10+++$

$$
\text { 10./2. - }
$$

15. $7 .-23 . / 3.7 \mathrm{Mk}$.

22./5.-21./6. $7 \mathrm{Mk}$.

24. $/ 3$. -

20./8.-19./9.7Mk. 16./8. -

28./9. 22./8. 1911 $20 . / 4$. -

24./11.-4./1. 27./11. +++ $10 \mathrm{Hg}$-Säckchen $15 . / 1.10+++$ à $1 \mathrm{~g}$ 40 Tage. 10./2. +++
$14 / 2$. - 25./3. dto. 24./3. +++

20./5.-29./6.dto.

à 2 g 40 Tage. $20 . / 5$. +++

20./8-29./9.dto $16 . / 8$. -

22./3.-6./5. dto. 22./3.11 +++ 25./4. $+t+$

Fall 78.

210/1909. Hulda Math. K. 28 Jahre. Dienstmädehen.

Inf.: 1906. Wahrscheinlich.

Partus: Im Juni 1909.

Stat. pr.: 26./8. 1909. Leukoderma;

0

Papelreste in der Vulva.

27./8. -

209/1909. Anna Elisabeth K.

Geboren: Im Juni 1906. Ausgetragen.

L. H. 45 .

Gewicht $2500 \mathrm{~g}$.

Stat. pr.: $\quad 26 . / 8.1909$. Koryza + Rhagad. oris + Exanth. pap. 
Fall 79.

228/1908. Agnes Charlotta K. 23 Jahre. Dienstmädchen. Inf.: Frühjahr 1905.

Stat. pr.: 24./10. 1905. Oedema durum $40+31 \mathrm{Hg}-$ + Pap. muc. vulv. et ani Säckchen à $8 g$ + Angina pap. + Exanth. $\quad+2$ Mk. pap. univ. + Psoriasis plant. + Leukoderma.

3./9. I906. Exanth. mac. pap. $40 \mathrm{Hg}-$ Säckchen

Partus: 15./6. 1908.

S tat. pr.: 2./9. 1908. Pap. serpig. et ulc. $\quad 2 . / 9 .+++$ trunci.

229/1908. Erik Alexander K.

Geboren: 15./6. 1908. Ausgetragen.

S-frei.

Stat. pr.: 2./9. 1908. Atrophie. Koryza + Sattelnase.

D.

Fal1 80.

141/1900. Emmy Charlotta A. 18 Jahre. Geschäftsgehilfin.

Inf.: Unbekannt.

Partus: 21./6. 1900.

Stat. pr.: $5 . / 9.1900$. Leukoderma.

137/1900. Sonja A.

L. H. 4 .

Geboren: 21./6. 1900.

Stat. pr.: 4./9. Exanth. mac. pap. univ.

Gewicht $4200 \mathrm{~g}$.

Fall 81 .

128/1908. Engla W. 28 Jahre. Köchin.

Inf.: Unbekannt.

Partus: $\quad$ 17./12. 1902.

S tat. pr.: 12./6. 1903. Leukoderma.

127/1903. Svea W.

L. H. 20.

Geboren: $17 . / 12.1902$.

Stat. pr.: 12./6. 1903. Pap. muc. vulv. et ani + Koryza.

Fall 82.

Edit Emilia B. 27 Jahre. Dienstmädchen.

Inf.: Unbekannt.

Partus: 22./5. 1903.

0

Stat. pr.: 18./7. 1903. Leukoderma.

Sven B.

I. H. 22 .

Geboren: 22./5. 1903.

Stat. pr.: 18./7. 1903. Exanth. pap. univ. + Koryza. 
Klin. Beobachtungen über die Prognose der kong. Syphilis. $\quad 159$

Fall 83.

32/1904. Amalia L. 24 Jahre. Dienstmädchen.

Inf.: Unbekant.

Partus: $\quad 28 . / 9.1903$.

Stat. pr.: $\quad 26 / 1$. 1904. Leukoderma.

33/1904. Gösta L. L. H. 23.

Geboren: $28 . / 9$. 1903 .

Stat. pr.: 26./1. 1904. Koryza + Pap. muc. ani.

Fall 84.

121/1904. Anna H. 34 Jahre. Fabriksarbeiterin.

Inf.: Unbekannt.

Partus: 28./5. 1904. 0

St at.pr.: 15./6. 1904. Psoriasis palm. + Leukoderma.

120/1904. Adolf $\mathrm{H}$.

L. H. 24 .

Geboren: $28 . / 5.1904$.

S tat. pr.: 15./6. 1904. Psoriasis palm. et plant. Gewicht $4300 \mathrm{~g}$.

Fall 85 .

Inf.: Anna Margareta B. 29 Jahre. Dienstmädchen.

Partus: 29.7. 1905.

Stat. pr.: 5./8. 1905. Roseola + Pap. muc. vulv. + Iseukoderma.
Josef B.
L. H. 30 .

Geboren: 29./7. 1905.

S tat. pr.: 5./8. 1905. Atrophie + Koryza.

Fall 86.

303/1908. Amalia 0. 35 Jahre. Näherin.

In f.: Unbekannt.

Partus : $\quad 24 . / 10.1908$.

Stat. pr.: 31./10. 1908. Erythema pap. univ. + Pap. muc. tons. + Lenkoderma.

304/1908. Nils 0 .

L. H. 42.

8

Geboren: $24 . / 10.1908$.

Stat. pr.: 31./10. 1908. Hepatitis.

Fal1 87.

104/1910. Maria Augusta A. 20 Jahre. Näherin.

In f.: Unbekannt.

Gravida: Seit Anfang August 1909 . 0

Partus: $\quad 8 . / 5.1910$. 
Stat. pr.: 27./5. 1910. Leukoderma. Narben an Labb. maj.

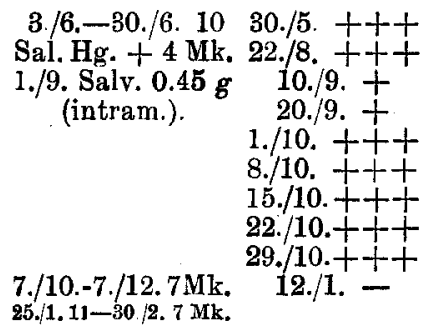

6./6. 1912. S-frei. W. R. - Während der Partus vor 2 Monaten; Kind letzten 2 Jahre ausgetragen, S-frei, W.R. - mehrere Inj.Kuren.

105/1910. Robert A

Geboren: $8 . / 5$. 1910. Ausgetragen. Ge-

L. H. 48. wicht $3700 \mathrm{~g}$. Erkrankt $26 . / 5$.

Stat. pr.: 27./5. Periostitides tibiae sin. et antibr. $\mathrm{dx}$.

30./5. +++

\section{Fall 88.}

73/1904. Maria H. 29 Jahre. Dienstmädchen.

Inf.:

Unbekannt.

Partus: $\quad 30 . / 1.1904$.

Stat. pr.: 12./3. 1904. Leukoderma.

72/1904. Arvid H.

Geboren: 30./1. 1904.

Stat. pr.: 10./3. 1904. Exanth. pap. univ. + Pap. muc. lab. sub. + Koryza.

Fall 89.

81/1908. Anna B. 24 Jahre.

Inf.: Unbekannt.

Stat. pr.: 7./9. 1908. Lenkoderma.

2./12. Leukoderma.

8./9.-5./10. 10 9./9. +++

Sal. Hg. +4 Mk. 14./10. -

2./12.-4./1. 1909 2./12. +++ $7 \mathrm{Mk}$.

$$
\begin{array}{cc} 
& 11 . / 1 .- \\
26 . / 2 .-5 . / 4.6 \mathrm{Mk} . & 14 . / 4 .- \\
19 . / 6 .-26 . / 7.7 \mathrm{Mk} . & 15 . / 6 .+++ \\
& 23 . / 7 .+++ \\
29 . / 9 .-10 . / 11 . & 29 . / 9 .+++ \\
7 \mathrm{Mk} . & \\
27 . / 1 .-1 . / 3.7 \mathrm{Mk} . & 24 . / 1 .+++ \\
13 . / 6 .-5 . / 8.7 \mathrm{Mk} . & 6 . / 4 .+++
\end{array}
$$

82/1908. Ernst B. $1 \mathrm{Jahr}, 8$ Monate.

Ausschlag ad.anum seit 3

Monaten. 
Klin. Beobachtungen über die Prognose der kong. Syphilis. 161

Stat. pr.: 7./9. 1908. Pap. muc. ani + 7./9.-17./10.Hg- 9./9. Roseola + Psoriasis palm. Säckchen40Tage $17 . / 10 .+++$ 2./12. +++ 11./1. 1909

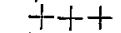

11./3. +++

14./4.

2./12. 1908-23./7, 15./6 $19096 \mathrm{Hg}$-Säck- 23./7. +++ chenkurenà1-2g. 29.19. +++

24./1. 1910

6. $1+$ t $^{+}+++$

E.

Fall 90 .

128/1909. Emma J. 27 Jahre. Köchin.

In f.:

Wahrscheinlich vor 7 Jahren; vor 6 Jahren Fehlgeburt im

7. Monate.

Partus: $\quad$ 8./1. 1909.

Stat.pr.: 19./5. 1909. S-frei.

124/1909. Sven Gustaf J.

Geboren: 8./1. 1909. Ausgetragen.

Stat. pr.: 19./5. 1909. Exanth. mac. pap. 20./5.-30./6. 24./5. +++ univ. + Rbagad. oris + Hg-Säckchen Koryza + Hepatitis + ̀̀ 1 g 40 Tage. Splenitis.

Fall 91.

72/1910. Anna Sofia P. 43 Jahre. Waschfrau.

In f.: $\quad$ Wabrscheinlich vor 7 Jahren;

0 Fehlgeburt im 5. Monat.

Partus: $\quad 12 . / 1.1910$.

Stat.pr.: $12 . /$. S-frei.

13./4. +++

71/1910. Tyra Sara P.

Geboren: 12./1. 1910. Gewicht $2500 \mathrm{~g}$.

Stat. pr.: 12./4. 1910. Koryza.

4./5. Albuminurie + Zylin-

0

drurie.

Stat. pr.: 27./7. Pap. faciei.

Fall 92.

485/1911. Hilda Elisabeth B. 42 Jahre. Ehefrau.

Inf.: $\quad$ Vor 26 Jahren Genitalgeschwüre, langwierige

Partus: $\quad$ 19./12. 1911

Stat. pr.: 29./12. 1911. Gummata ulcer. KJ. $1-2 \times 3.29 . / 12 .+++$ trunci et brach. sin. + Periostitis clav. et hum. sin. + Nephritis chr. + Ascites.

Archiv f. Dermat. u. Sypk. Bd. CXVI. 
486/1911. Anna Elisabeth B.

Geboren: 19./12. 1911. Ausgetragen.

Stat. pr.: 29./12. Atrophie + Erosion.

29. $12 .+++$ ani + Hepat. + Splenitis.

Fall 93.

22/1910. Karl Johann K.

Geboren: 9./1. 1910.

Die Mutter: Inf. mehr als 10 Jahre zurück, jetzt Lues cerebri.

St at. pr.: 20./1. 1910. S.frei.

$40 \mathrm{Hg}$-Säckchen 20./1. $10++$ à $1 / 2 \mathrm{~g}-6 . / 3$.

$21 . / 4 .-30 . / 5$.

15./4. -

$40 \mathrm{Hg}$-Säckcher

à $1 \mathrm{~g}$.

Mors 12./10, in Nephritis.

25./8. -29./9.dto. 29./9. -

Fall 94.

Ida Mathilda S. 35 Jahre.

Inf: $\quad 1906$.

Partus I: Juni 1907. Lues hereditaria.

$$
\text { Mors 3./8. } 1907 .
$$

Stat.pr.: 26./7. 1907. Leukoderma.

Partus II: 30/8. 1910.

Hg-Säckchen-

Kur.

Sonja S.

Geboren: $30 . / 9.1910$.

Stat. pr.: 10/10. 1910. Atrophie + Koryza.

Mors 1./8. 1911 in Kapillarbronchitis.

F all 95.

299/1909. Tyra Lovisa J. 23 Jahre. Zigarrenarbeiterin.

Gravida: Mars 1905.

Inf.: Juni 1905.

Stat. pr.: 1./9. 1905. Sklerose + Adenitis.

Partus I: 14./12. 1905. Das Kind: Ge-

L. H. 59 .

10./10. 1910

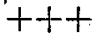

Hg-Säckchen

à 8 g 40 Tage. 1906-1907 wicht $3500 \mathrm{~g}$. Ausgetragen. $5 \times 40 \mathrm{Hg}$-Säck$3 \mathrm{Hg}$-Säckchenkuren erhal- chen à $8 \mathrm{~g}$. ten, niemals luetische Symptome gezeigt, aber am 22./12. 1909 W. R. +++

Partus II: Januar 1909. Ein Monat zu frük. Nach einem Monat an einer Darmerkrankung gestorben.

Partus III: 8./12. 1909.

Stat. pr.: $20 . / 12,1909$. S-frei. 
300/1909. Sven Wilh. J.

Geboren: 8./12.1909. Ein Monat zu früh.

Stat. pr.: 20./12. Exanth. pap. univ. + Atrophie.

23./12. Mors.

$22.12 .+++$

Fal1 96.

In f.:

4/1910. Selma D. 30 Jahre. Dienstmädchen.

Partus I: Im März 1904.

Stat. pr.: $28 . / 3$. 1904. Leukoderma $+40+40+36$

Pap. corporis.

Partus: $\quad$ 13./9. 1909 .

Stat. pr.: 7.|1. 1910. S-frei.
$\mathrm{Hg}$-Säckchen.

Seitdem 0.

7./1.-15./2.

9 Asurol +

1 Huile grise. 14./2. +++

5/1910. Ernst Uno D.

Geboren: 13./9. 1909. 1 Monat zu früh. $11 / 1 .-27 . / 1$.

S tat. pr.: 7./1. 1910. Koryza + Exanth. Hg-Säckchen

$8 . / 1 .++$ papul. univ. + Hepatitis + à $0.5 \mathrm{~g}$.

Pap. muc. ani.

5./2.Mors in Bronchopneumon.

Fall 97.

232/1909. Anna Mary W. 38 Jahre. Ehefrau.

Inf.: $\quad 1897$.

S tat. pr.: 7./2. 1898. Pap. muc. vulv, et Hg:Säckchen faucium + Leukoderma. à $6 \mathrm{~g} 40$ Tage. 1899. Totgeborene Frucht 7 Monate alt.

1900. Totgeborene Frucht 8 Monate alt.

1901. Totgeborene Frucht 8 Einige HgMonate alt. Behandlungen.

1904. Ausgetragenes Kind, das noch lebt, gesund (?)

Stat. pr.: 28./9. Tabes incipiens.

29./9. +++

11./10.-17./11.

5./1. 1910-18./2. 13./1. 1910

$7 \mathrm{Mk}$.

25. $/ 5 .-13 . / 7$.

$7 \mathrm{Mk}$.

24:/8.-12./10.

$7 \mathrm{Mk}$.

14./12.-1./2. 28./12. +++

233/1909. Eivor Alice W.

$19117 \mathrm{Mk}$.

$+++$

25. $/ 5 .++$

$3: / 8$. +++

Geboren: 6./9. 1909.

Stat. pr.: 28./9.1909. Exanth. mac. pap. univ. + Koryza + Rhagad. oris.

1./11. 1909. Mors in Nephritis.
29./9.-1./11.

29./9. +++

Hg-Säckchen

à $1 / 2$ g 32 Tage. 
Fall 98.

13/1911. Anna Maria S. 34 Jahre. Ehefrau.

Inf." $\quad 1898$.

Gravida: Gleichzeitig.

Stat. pr.: 19./7. 1898. Roseola + Pap. Hg-Säckchen muc. vulv. à $5 g 40$ Tage.

Partus: 20./10. 1898. Das atrophische Hg-Säckchen Kind hatte Koryza und La- à $6 \mathrm{~g} 40$ Tage. ryngitis und starb $1 \mathrm{Jahr} 190640 \mathrm{Hg}$ und 1 Monat alt an Cho- Säckchen. lera infantum.

Partus: $\quad 23 . / 10.1910$.

Stat. pr.: $9 . / 1,1911$. S-frei.

19084 Inj.

14/1911. Bertha Maria S.

$$
\begin{gathered}
\text { 19./1. } \frac{27 . / 1}{3 \mathrm{Mk} .} \\
\text {. }
\end{gathered}
$$

Geboren: $23 . / 10$. 1910. S-frei

Stat. pr.: 7./1. 1911. Exanth. mac. pap. univ.

11./2. Mors.

7./1.- $-11 . / 2$.
Hg-Säckchen

11. $1 .+++$

à $1 \mathrm{~g} 31$ Tage.

Fall 99.

39/1912. Vera Maria K. 6 Monate,

Geboren: 6./8. 1911. Ausgetragen.

Anamnese Fall Nr. 13 .

Stat.pr.: 30./1. 1912. Pap. muc. ani.

Koryza. Hepatitis. Splenitis.

30./1. 1912

Fall 100.

25/1907. Anna E. 34 Jahre. Näherin.

Inf.: $\quad$ Vor. 6 Jahren.

Stat. pr.: 25./9. 1906. Ule. gummosa + Pap. tubercul. + Leukoderma.

Partus: $\quad$ 18,/10. 1907.

2 Schmierkuren.

$$
\begin{gathered}
\text { Hg-Säckchen } \\
\dot{a} 8 \mathrm{~g} \\
40+40 \text { Tage. }
\end{gathered}
$$

26/1907. Karin E.

L. H. 33 .

Geboren: 18./1. 1907 .

Stat. pr.: 4./2. 1907. S-frei.

Fall 101.

212/1911. Gertrud Bernhardina C. 33 Jahre. Kellnerin.

Inf.:

Grühjahr 1907. 0

Stat.pr.: 5./10.1908. Leukoderma + 5/10.-16./11. Pigmentflecke nach Pap. Hg-Säckchen
muc. vulv.

Gravida: Oktober 1910

Stat. pr.: 6./12. 1910. S-frei.

8./12.-27./2. II.

16./11. 1908

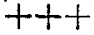

7./12. $1910-$ 
Klin. Beobachtungen über die Prognose der kong. Syphilis. 165

Partus: $\quad 9 . / 6.1911$.

Stat.pr.: 17./6. 1911. S-frei. 19./6. $1911+$

213/1911. Astrid Margaretha C.

Geboren: 9./6. 1911. Ausgetragen.

Gewicht $3150 \mathrm{~g}$.

Stat. pr.: 17./6. 1911. S-frei.

\section{Fall 10?.}

113/1902. Frida Maria S. 29 Jahre. Ehefrau.

Inf.: Mitte 1891.

Stat. pr.: 13./11. 1891. Pap. muc. vulv. tons. + Psoriasis plant. 2 Inj.-Kuren.

3 Schmierkuren.

2 Pillenkuren

Partus I: 9./12. 1891.

1891-1896.

Partus II: Abort im 3. Monat.

Gravida: Seit Juli 1901.

Stat. pr.: 6./3. 1902. Pap. muc. vulv.

Partus: 22./4. 1902.

L. H. 62 .

Stat.pr.: 2./5. 1902. S-frei.

114/1902. Martin S.

Geboren: 22./4. 1902 .

Stat. pr.: 2./5. 1902. S-frei. Gewicht $4340 \mathrm{~g}$.

Hg-Säckchen à 8 g 40 Tage.

L. H. 15.

\section{Fall 103 .}

490/1911. Maj Ingeborg S. 3 Jahre.

Die Mutter inf. 1901.

Partus I: Kind mit Lues hereditaria. Hg-Behandlung.

Partus II: Gesunder Kind.

Partus III: Gestorben im Alter von $2^{1 / a}$ Monaten an Lues heredit.

Partus IV: = Pat. 5 Monate alt. Pseudo-

paralyse des rechten Armes. Hg. c. creta.

Stat. pr.: 7./11. 1911. Pap. muc. hypertroph. ad anum. 


\section{Gruppe III.}

Fall 104.

Augusta F.

Stat. pr.: 11./11.1910. Roseola + Pap. muc, vulv.

Partus: $\quad$ 7./12. 1910.

$14 . / 11,0.5 \mathrm{Sa}:-$ varsan $I$.

Stat. pr.: $\underset{\substack{\text { Koryza } \\ \text { nitis. }}}{\text { Erik }}+$ Hepatitis + Sple-

L. H. 52.

11./1. $11-$

Fall 105.

193/1900. Hulda L. 18 Jahre.

Partus: 11./11. 1900.

Hg-Kur.

Stat. pr.: 19./11. 1900. S-frei.

194/1900. Gulli I.

I. H. 7 .

Geboren: 11./11. 1900.

Stat. pr.: 19./11. 1900. S-frei.

Fall 106.

149/1903. Anna Maria S. 33 Jahre. Fabriksarbeiterin.

Inf,: Unbekannt.

Partus: 9./2. 1903.

S tat. pr.: 21.7.1903. Cicatrices vulv. + Polyadenit.

147/1903. Karl S.

Geboren: 9./2. 1903 .

L. H. 18 .

Stat. pr.: 20./7. 1903. Exanth. pap. univ. + Pap. muc. ad anum + Koryza.

Fall 107.

457/1911. Viktoria B. 21 Jahre. Geschäftsgehilfin.

Inf: $\quad$ Unbekannt.

Partus: 21./11. 1911.

S tat. pr.: 1./12. 1911. Pap. muc. tons. +

Papelreste in der Vulva. + Adenitis.

2./2. $t+t$

458/1911. Karl Olof B.

Geboren: 21./11. 1911 .

Stat. pr.: 1./12. S-frei.

2./2. -

Fall 108.

Ester J. 25 Jahre. Dienstmädchen.

Inf.: Unbekannt. 
Partus. 30.1. 1908.

Stat. pr.: Februar 1908. Lues Cerebri.

Tyra J.

Geboren: 30./3. 1908. S-frei.

L. H. 39 .

Fall 109.

225/1909. Anna Linnéa J. 30 Jahre. Ehefrau.

Inf.: Unbekannt.

Partus I: Dez. 1907. Ein totgeborenes

Partus II: $16 . / 6.1909$

Kind in dem 7. Monat.

Stat. pr.: $23 . / 9$. S-frei.

Gravida: Seit Mai 1910.

Partus III: 1./10. Ausgetragen. S-frei. Gewicht 3.900 $g$.

30./9.-5./11, $1024 . / 9 .+++$

Sal. Hg. $+4 \mathrm{Mk}$. 15./11. -

3./1. $1910-16 . / 2$.

$7 \mathrm{Mk} . \quad 16 . / 2.1910$ -

24./3.-2./5.7Mk. $5 . / 4$. -

4./7.-18./8.7 Mk.

1./7. -

$9 / 11 .-22 . / 12$.
7. Mk.

2./9. -

28./12. -

226/1909. Gustav Algot J.

Geboren: 16./6. 1909. Ausgetragen.

Gewicht $2 \cdot 600 \mathrm{~g}$.

Stat. pr.: 23./9.1909. Atrophie + Koryza 27./9. Hg.Säck- 24./9. +++ + Nephritis.

Mors 10./10 1909 in Nephritis. chen $\dot{a} 1 \mathrm{~g}$

5 'Tage.

Fal1 110.

337/1908. Wilhelmina Sofia 0. 27 Jahre. Ehefrau.

Inf.:

Unbekannt.

Seit 1903 IV. Partus: 2 totgeboren und 2 tote bei jungem Alter.

Partus V: 23./11. 1908.

Stat. pr.: 1./12. 1908. S-frei.

Partus VI: 14./6. Ansgetragen: S-frei.

$$
\begin{aligned}
& \text { 10./12.-18./1. } 10 \\
& \text { Sal. Hg 21./1. } 1909- \\
& \text { 24. } / 2 .-7 . / 47 \mathrm{Mk} \text {. } \\
& \text { 2./6.-28./7: } 7 \text { Mk. 2./6. }+++ \\
& \text { 22./9.-3./11.7Mk. } \\
& \text { 8./9. }+++ \\
& \text { 29./12.-9./2.7Mk. } \\
& \text { 23./3.-11./4.Hg- } \\
& \text { Säckchen à } 8 g \\
& 20 \text { Tage } \\
& \text { 20./4.-30./5.dto. } \\
& 40 \text { Tage } \\
& \text { Fortgesetzte } \\
& \text { Säckchenbe- } \\
& \text { handlung. } \\
& \text { 2./12. }+++ \\
& \text { 3./11. } \\
& \text { 9./2. } 1910- \\
& \text { 23./3. - } \\
& \text { 1./6. - } \\
& \text { 5./7. - } \\
& \text { 25./1. } 1911-
\end{aligned}
$$


336/1908. Marie Wilhelmina Elisabeth. 0 .

Geboren: 23./11. 08. Ein Monat zu früb.

Stat. pr.: 1./12. 1908. Pemphigus syphi-

2./12. +++ liticus + Exantb. mac. pap.

Mors. 12./12.

Fall 111.

Hanna Charlotta K. 25 Jahre.

Inf: $\quad$ Unbekannt.

0

Partus: $\quad 13.77 .1910$.

Stat.pr.: 12./8. 1910. S-frei.

12./8. $+t+$

Astrid K.

Geboren: $13 . / 7,1910$.

Stat. pr.: 12./8. Koryza + Exanth. + pap. muc. ani + Splenitis.

12./8. +++

Fall 112.

283/1910. Matti Klara H. 28 Jahre. Näherin.

In f.: Ist verleugnet.

Stat. pr.: 26./11. 1910. S-frei.

28./11. +++

283/1910. Matts $\mathrm{H}$.

L. H. 57.

Geboren: 14./6. 1910.

Stat. pr.: 26./11. Pap. frontis.

Fall 113.

114/1911. Amalia J. 23 Jahre. Näherin.

Inf.: $\quad$ Wahrscheinlich v. $2^{1} / 2$ Jahren.

Partus: $\quad$ 19./2.1911.

Stat. pr.: 30./3. 1911. S-frei.

1./4. +++

115/1911. Anders Roland J.

Geboren: 19./2. 1911. Ausgetragen.

Gewicht $2 \cdot 300 \mathrm{~g}$.

Stat. pr.: 30./3. 1911. Psoriasis palm. et

0

L. H. 55. plant. + Pap. ani. + Rhagad. oris.

L. H. 61 .

Fall 114.

153/1909. Gustafva J. 37 Jahre. Wäscherin.

In f.: Unbekannt.

Ein Knabe 6 Jahre, gesund.

Partus II: Vor 8 Monaten.

Stat. pr.: 17./6. 1909. S-frei.

22./6. $-20 . / 7.10$

18./6. $+t+$

Sal. Hg. $+4 \mathrm{Mk}$.

2./9. - 8./10.7Mk. 18./10. +++

30./3. -

$7.77+++$ 2./9. +++

152/1909. Sven Gustav J. 8 Monate.

Geboren: Ausgetragen.

Ausschlag Mai 1909. 
Klin. Beobachtungen über die Prognose der kong. Syphilis. 169

Stat. pr.: 17./6.1909. Exanth. mac. pap. univ.

18./6. +++

22./6.-31./7. $\mathrm{Hg}$ -

Säckchen à $1 \mathrm{~g}$

7./7.

1./9. +++ 40 Tage

8./9.-17./10. dto. 19./10. +++

F.all 115 .

268/1909. Elna Viktoria Svea N. 22 Jahre. Friseur.

Inf.: Unbekannt.

Partus: $\quad$ 3./11. 1909.

Stat. pr.: 10./11. 1909. S-frei.

$$
\begin{aligned}
& \text { 19./11.-16./12. } \\
& 10 \text { Sal. Hg. } \\
& +4 \mathrm{Mk} .
\end{aligned}
$$

17./12. -

269/1909. Lave Sven Waldemar N.

Geboren: 3./11. 1909. Ein Monat zu früh.

Stat. pr.: $\quad 10 . / 11.1909$. Pemphigus syphiliticus + Exanth. papul. + Koryza.

13./11. Mors.

Fall 116.

Mutter und eine Schwester sollen W. R. +++ haben.

Helmer J.

Geboren: 25./6. 1907 .

Meningitis luetica.

S tat. pr.: $19 . / 3$. 1910. S-frei.

Fall 117.

Sven Axel Arne $L$.

$191 / 1911$.

Mutter: Soll Lues haben.

Geboren: $12 . / 8$. 1910.

Stat. pr.: Nov. 1910. Exanthema univ.

L. H. 54 .

Hg-Kur.

Fall 118.

Mutter unbekannt.

Doris C.

L. H. 58 .

Geboren: 29./11. 1908 .

Stat.pr.: 8./5. 1909. Rhagad oris + Exanth. papulos.

Fall 119.

Inf.: Unbekannt.

Partus: $\quad 27 . / 3$. 1901 .

Stat.pr.: 22./7. 1902. S-frei.

0

192/1902. Astrid F.

Geboren: $27 . / 3.1901$.

Stat.pr.: 22./7. 1902. Pap. muc. ani + Erosiones oris.
L. H. 60 .

5./7. +++ 
Fall 120.

Johanna Maria K. Ehefrau.

Inf.: Verneint.

Partus: $\quad 8.110 .1901$.

Stat. pr.: 12./12. S-frei.

243/1901. Nils $N$.

Geboren: 8./10. 1901.

I. II. 10.

Stat. pr.: 12./12. 1901. Exanth. pap. + Rhagad oris.

Fall 121.

Inf: : Unbekannt.

S-frei.
Wivi E.
L. H. 49 .

Geboren: 29./9. 1909 .

Stat. pr.: Exanth. pap. univ.

Fall 122.

313/1907. Gerda B.

Geboren: 21./12. 1907 .

Von der Mutter keine Angaben.

Stat.pr.: $\quad 31 . / 12.1907$. Exanth. pap.mac. univ. + Koryza.

Fall 123.

179/1908. Sigrid Olivia L.

Geboren: 22./5. 1908.

Von der Mutter keine Angaben.

Stat. pr.: $\quad$ 10./7. 1908. Exanth. mac. pap. 11./7-19./8. Hguniv. + Rhagad oris. Säckchen à $1 / 2 g$

40 Tage.

21./9.-1./11.dto.

à $1 \mathrm{~g} \quad 40$ Tage

14./1.1909-4./3.

9./11. -

dto. à $2 g 40$ Tage

6./7.-14./8. dto.

12./3. -

8./4. -

19./8. -

Fall 124.

195/1909. Bertha J. 2 Jahre.

Die Mutter unbekannt.

In Juni 1909 luetischer Ausschlag in den Plantae und Palmae wie auch epileptiforme Anfälle. 
Klin. Beobachtungen über die Prognose der kong. Syphilis, 171

Stat. pr.: 4./8.1909. Sattelnase, übrigens $17 . / 8 .-25 . / 9 . \mathrm{Hg}-$ S-frei.

Säckchen à $2 g$

$$
40 \text { Tage. }
$$

25./10-3./12. dto.

28./9. +++
27./10. +++
3./12. +++

Fall 124.

434/1911. Vera Viola K.

Geboren: 10:/9. 1911.

Von der Mutter nichts bekannt.

(Der Vater wurde vor 4 Jahren für Syphilis gepflegt.)

Seit 14 Tagen in dem rechten Kniegelenk erkrankt.

Stat. pr.: 16./11. Osteochondritis fem 10./11. +++ dx. luetica + Exanth. maculo papulosum.

Mors 15./12.

Fall 126.

203/1911. Karl Gustav E.

Geboren: 1./5. 1911.

Die Mutter hat 2 Frühgeburten in resp. dem 5. und 6. Monate gehabt.

Stat. pr.: 9./6.1911. Exanth. mac. pap. univ. + Koryza + Rhagad. oris. + Atrophie.

23./7. Mors in Pneumonie.

9./6.-22./6. $\mathrm{Hg}$. Säckchen à $0.5 \mathrm{~g}$ 13 Tage.

27./6. Salvarsan

0.3 (Intram).

Fall 127 .

Eltern unbekannt.

17\%/1900. Anna Lisa A.

L. H. 5 .

Geboren: $12 . / 8.1900$.

S tat. pr.: 2./11. 1900. Pap. + Koryza + Rhagad ani. 


\begin{tabular}{|c|c|c|c|c|c|c|}
\hline \multirow{3}{*}{$\mathbf{N r}$} & \multirow{3}{*}{$\begin{array}{l}\text { Na m e } \\
\text { und } \\
\text { Geburtstag }\end{array}$} & \multicolumn{5}{|c|}{$M$ u $t$ t e r } \\
\hline & & \multirow{2}{*}{$\begin{array}{l}\text { Alter der } \\
\text { Syphilis }\end{array}$} & \multicolumn{2}{|c|}{ Be $\mathrm{h}$ a nd l ung } & \multirow[b]{2}{*}{ Brscheinungen } & \multirow[b]{2}{*}{ Serorealstion } \\
\hline & & & $\begin{array}{l}\text { vor der } \\
\text { Gravidität }\end{array}$ & $\begin{array}{l}\text { während der } \\
\text { Graviditãt }\end{array}$ & & \\
\hline 1 & $\begin{array}{l}\text { Wera W. } \\
28 / 101899\end{array}$ & $\begin{array}{l}\text { Ungefähr } \\
\mathbf{3} / 4 \mathbf{J a h r}\end{array}$ & 0 & $\begin{array}{l}\text { Merkuriolsäck- } \\
\text { chen ì } 5 \mathrm{~g} \\
40 \text { Tage }\end{array}$ & $\begin{array}{l}\text { Sklerosereste, } \\
\text { Pap. muc. vulv., } \\
\text { Pap. diss. }\end{array}$ & . \\
\hline 2 & $\begin{array}{l}\text { Erik Ch. } \\
31 / 51899\end{array}$ & $\begin{array}{l}\text { Wahrscheinlich } \\
\text { 1 Jahr }\end{array}$ & 0 & 1 Schmierkur & $\begin{array}{l}\text { !'soriasis plan- } \\
\text { taris, Leuko- } \\
\text { derma }\end{array}$ & $\cdot$ \\
\hline 3 & $\begin{array}{l}\text { Wilhelm A. } \\
2 / 7 \\
1900\end{array}$ & 4 Monate & 0 & 0 & $\begin{array}{c}\text { Sklerose } \\
\text { adenit., Roseola }\end{array}$ & $\cdot$ \\
\hline 4 & $\begin{array}{l}\text { Sonja A. } \\
21 / 61900\end{array}$ & $\begin{array}{l}\text { Infektion } \\
\text { unbekannt }\end{array}$ & 0 & 0 & Leukoderma & $\cdot$ \\
\hline 5 & $\underset{12 / 8}{\operatorname{Anna-Lisa} A} \mathrm{~A}$ & \multicolumn{4}{|c|}{ Eltern unbekannt } & \\
\hline 6 & $\underset{8 / 91900}{\text { Karl Oskar K. }}$ & $\begin{array}{c}\text { Wahrscheinlich } \\
3 / 4 \mathrm{Jahr}\end{array}$ & 0 & 0 & Pap. muc. vulv. & $\cdot$ \\
\hline 7 & $\begin{array}{l}\text { Gulli L. } \\
11 / 111900\end{array}$ & $\begin{array}{l}\text { Infektion } \\
\text { unbekannt }\end{array}$ & Unbekannt & $\mathrm{Hg}$-Kur & $\begin{array}{c}\text { Soll sichere } \\
\text { syphilitische } \\
\text { Symptome auf- } \\
\text { gewiesen haben }\end{array}$ & $\cdot$ \\
\hline 8 & $\begin{array}{l}\text { Elsa E. } \\
7 / 6 \\
1901\end{array}$ & $\begin{array}{l}\text { Ungefähr } \\
8 \text { Monate }\end{array}$ & 0 & $\begin{array}{l}\text { Hog-Säckchen } \\
\text { à } 6 \text { g } 40 \text { Tage }\end{array}$ & $\begin{array}{c}\text { Sklerose, Roseola } \\
\text { Pap. muc. vulv. } \\
\text { et ani }\end{array}$ & $\cdot$ \\
\hline 9 & $\begin{array}{l}\text { Sven B. } \\
14 / 61901\end{array}$ & $\begin{array}{l}\text { Ungefähr } \\
1 \text { Jahr }\end{array}$ & 0 & $\begin{array}{c}\text { Hg-Säckchen } \\
\text { à } 6 \underset{g}{40+40}+40 \\
\text { Tage }\end{array}$ & $\begin{array}{l}\text { Pap. muc. vulv., } \\
\text { Exanth. mac., } \\
\text { Pap. univers., } \\
\text { Leukoderma, }\end{array}$ & $\cdot$ \\
\hline 10 & $\begin{array}{l}\text { Nils N. } \\
8 / 101901\end{array}$ & $\begin{array}{l}\text { Infektion } \\
\text { verneint }\end{array}$ & 0 & 0 & S-frei & $\cdot$ \\
\hline 11 & $\begin{array}{l}\text { Ernst J. } \\
4 / 21902\end{array}$ & 2 Jahre & $\begin{array}{c}H g-\text { Säckchen } \\
\text { à } 6 \text { g } 40+40+40 \\
\text { Tage }\end{array}$ & $\begin{array}{c}\mathrm{Hg} \text {-Säckchen } \\
\text { à } 6 \text { g } 37 \text { Tage }\end{array}$ & $\begin{array}{c}\text { Pap. muc. vulv., } \\
\text { Leukoderma }\end{array}$ & $\cdot$ \\
\hline 12 & $\underset{18 / 5}{\operatorname{Karl} A}$ & 7 Monate & 0 & $\begin{array}{c}\text { Hg-Säckchen } \\
\dot{a} 7 \mathrm{~g} 19 \text { Tage }\end{array}$ & $\begin{array}{l}\text { Sklerose, Oedema } \\
\text { dur. pap. trunci, } \\
\text { Roseola }\end{array}$ & • \\
\hline 13 & $\begin{array}{l}\text { Astrid F. } \\
27 / 3 \quad 1901\end{array}$ & $\begin{array}{l}\text { Infelation } \\
\text { wnbekannt }\end{array}$ & 0 & 0 & $S-$ frei & $\cdot$ \\
\hline
\end{tabular}




\begin{tabular}{|c|c|c|c|c|c|c|}
\hline \multicolumn{6}{|c|}{$\begin{array}{llll} & i & n & d\end{array}$} & \multirow{3}{*}{ Bemerkungen } \\
\hline \multicolumn{2}{|c|}{ Bei der Geburt } & \multicolumn{4}{|c|}{$V$ e r 1 a u f } & \\
\hline Symptome & $\begin{array}{c}\text { Sero- } \\
\text { reaktion }\end{array}$ & I. Jahr & II. Jahr & III. Jahr & IV. Jahr & \\
\hline $\begin{array}{c}\text { S-frei } \\
2^{\circ} 650 \mathrm{~g}\end{array}$ & . & 0 & $\begin{array}{c}0 \\
\text { Rubeola }\end{array}$ & $\begin{array}{c}0 \\
\text { Pneumonie }\end{array}$ & 0 & $\begin{array}{l}\text { 20/12 1906. Keine Erschei- } \\
\text { nungen. Geht in die Schule. } \\
\text { Juni 1912. Gut entwickelt. } \\
\text { S-frei. }\end{array}$ \\
\hline $\begin{array}{c}\text { Atrophie, } \\
\text { Impetigo } \\
\text { cont. }\end{array}$ & - & 0 & $\begin{array}{c}0 \\
\text { Rubeola }\end{array}$ & $\stackrel{0}{\text { Morbilli }}$ & 0 & $\begin{array}{l}\text { Keine Erscheinungen. } \\
\dagger \text { durch Unfall } 1905 .\end{array}$ \\
\hline $\begin{array}{c}\text { S-frei } \\
\mathbf{3} 700 \mathrm{~g}\end{array}$ & · & $\begin{array}{c}0 \\
\text { Pneumonie }\end{array}$ & 0 & 0 & 0 & $\begin{array}{l}\text { 18/\% 1906. Gut entw. S-frei. } \\
\text { Juni 1912. Sehr gut entw. } \\
\text { S-frei. }\end{array}$ \\
\hline $\begin{array}{l}\text { Exanth., } \\
\text { Mac.pap. } \\
\text { univ. } 4-200 g\end{array}$ & $\cdot$ & 0 & 0 & 0 & 0 & 29/7 1905. S-frei. Gut entw. \\
\hline $\begin{array}{c}\text { Papulae, } \\
\text { Rhag. aui, } \\
\text { Koryza }\end{array}$ & $\cdot$ & $\begin{array}{l}0^{\mathbf{3 1}} / \mathrm{s} 1901 \mathrm{in} \\
\dagger_{\mathrm{s}} \\
\text { Pneumonie }\end{array}$ & $\cdot$ & - & . & $\begin{array}{l}\text { Bei der Sektion keine } \\
\text { Zeichen von Syphilis. }\end{array}$ \\
\hline Ikterus & . & $\begin{array}{c}0 \\
\text { Pneumonie }\end{array}$ & 0 & $\underset{\text { Morbilli }}{0}$ & 0 & - \\
\hline $\begin{array}{c}\text { S-frei } \\
2.950 \mathrm{~g}\end{array}$ & $\cdot$ & $\begin{array}{c}02 / 81901 \text { in } \\
+\quad \text { Gastroente- } \\
\text { ritis }\end{array}$ & $\cdot$ & $\therefore$ & • & $\begin{array}{l}\text { Bei der Sektion keine } \\
\text { Zeichen von Syphilis. }\end{array}$ \\
\hline S-frei & . & 0 & 0 & 0 & 0 & $\begin{array}{l}{ }^{30} / 121906 . \text { S-frei. } \\
\text { Juni } 1912 . \text { Sehr gut ent- } \\
\text { wickelt. S frei. }\end{array}$ \\
\hline $\begin{array}{l}\text { S-frei } \\
3^{*} 180 \mathrm{~g}\end{array}$ & • & 0 & $\stackrel{0}{\text { Morbilli }}$ & $\begin{array}{c}0 \\
\text { Diphtherie }\end{array}$ & 0 & $\begin{array}{l}\text { 16/4 1910. S-frei. } \\
\text { W. R. - }\end{array}$ \\
\hline $\begin{array}{l}\text { Exanth. } \\
\text { pap., } \\
\text { Rhagad. } \\
\text { oris }\end{array}$ & $\cdot$ & 0 & 0 & $\begin{array}{c}0 \\
\text { Diphtherie }\end{array}$ & 0 & $\begin{array}{l}\text { 6/10 1909. S-frei. W. R. - } \\
\text { Juni 1912. S-frei. } \\
\text { Der Knabe ist begabt. }\end{array}$ \\
\hline $\begin{array}{c}\text { S-frei } \\
3.470 \mathrm{~g}\end{array}$ & • & 0 & $\begin{array}{c}0 \\
\text { Morbilli }\end{array}$ & $\begin{array}{c}0 \\
\text { Diphtherie }\end{array}$ & $\stackrel{0}{\text { Varicellae }}$ & - \\
\hline $\mid \begin{array}{l}\text { Psor. plant., } \\
\text { Koryza } \\
3.150 \mathrm{~g}\end{array}$ & $\cdot$ & $\begin{array}{c}0 \\
\text { Morbilli }\end{array}$ & $\begin{array}{c}0 \\
\text { Diphtherie }\end{array}$ & $\begin{array}{c}0 \\
\text { Pneumonie }\end{array}$ & 0 & - \\
\hline $\begin{array}{l}\text { Pap. muc. } \\
\text { ani }+, \\
\text { Erosiones } \\
\text { oris }\end{array}$ & $\cdot$ & 0 & 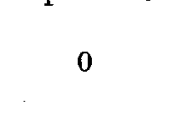 & - & 0 & $\begin{array}{l}\text { 1906. Kerat. parenchymat. } \\
15 / 2 \text { 1908. S-frei. } \\
\text { 28/6 1912. S-frei. W.R. - }\end{array}$ \\
\hline
\end{tabular}




\begin{tabular}{|c|c|c|c|c|c|c|}
\hline \multirow{3}{*}{ Nr. } & \multirow{3}{*}{$\begin{array}{l}\text { Na m e } \\
\text { und } \\
\text { Geburtstag }\end{array}$} & \multicolumn{5}{|c|}{$M u t t e r$} \\
\hline & & \multirow{2}{*}{$\begin{array}{l}\text { Alter der } \\
\text { Syphilis }\end{array}$} & \multicolumn{2}{|c|}{ Beband I ung } & \multirow[b]{2}{*}{ Erscheinungen } & \multirow[b]{2}{*}{ Seroreaktion } \\
\hline & & & $\begin{array}{c}\text { vor der } \\
\text { Graviditatit }\end{array}$ & $\begin{array}{l}\text { während der } \\
\text { Gravidität }\end{array}$ & & \\
\hline 14 & Hildur $\mathbf{K}$. & & Lues acquisita & & & . \\
\hline 15 & $\underset{22 / 4}{\text { Martin S. }}$ & 11 Jahre & $\left|\begin{array}{c}2 \text { Injektionskur., } \\
3 \text { Schmierkuren, } \\
\text { 2 Pillenkuren } \\
1891-1896\end{array}\right|$ & $\begin{array}{l}\text { Hg-Säckchen } \\
\text { à } 8 \text { g } 40 \text { Tage }\end{array}$ & $\begin{array}{c}\text { Pap, muc. vulv, } \\
\text { Leukoderma, } \\
\text { Cephalalgia, } \\
\text { Abort } 1899\end{array}$ & • \\
\hline 16 & $\begin{array}{l}\text { Harriet L. } \\
15 / 21902\end{array}$ & $2^{1} / 2$ Jahre & 0 & 0 & S-frei & • \\
\hline 17 & $\begin{array}{l}\text { Linnea J. } \\
17 / 21903\end{array}$ & 4 Monate & 0 & 0 & $\begin{array}{c}\text { Pap. muc. vulv., } \\
\text { Leukoderma, } \\
\text { Roseola }\end{array}$ & - \\
\hline 18 & $\begin{array}{l}\text { Karl S. } \\
9 / 21903\end{array}$ & $\begin{array}{c}\text { Infektion } \\
\text { unbekannt }\end{array}$ & 0 & 0 & $\begin{array}{c}\text { Cicatrites vul- } \\
\text { vae, Polyadenit. }\end{array}$ & $\cdot$ \\
\hline 19 & $\underset{28 / 3}{\text { Gustav N. }} \mathbf{N}$ & $1^{1 / 2} \mathrm{Jahr}$ & $\begin{array}{c}\text { Hg-Säckehen } \\
\text { à } 8 \text { g 40 Tage, } \\
\text { Hg-Säckchen } \\
\text { à } 8 \text { g } 15 \text { Tage } \\
100 \text { Hg-Pillen }\end{array}$ & 0 & $\begin{array}{l}\text { Pap. muc. vulv, } \\
\text { et ani, } \\
\text { Leukoderma }\end{array}$ & $\cdot$ \\
\hline 20 & $\begin{array}{l}\text { Svea W. } \\
17 / 121902\end{array}$ & $\begin{array}{c}\text { Infektion } \\
\text { unbekannt }\end{array}$ & 0 & 0 & Leukoderma & . \\
\hline 21 & $\underset{\text { s0 } / 1}{\text { Margit } X .}$ & $\begin{array}{c}\text { Infektion } \\
\text { unbekannt }\end{array}$ & 0 & 0 & Leukoderma & . \\
\hline 22 & $\begin{array}{l}\text { Sven B. } \\
22 / 51903\end{array}$ & $\begin{array}{c}\text { Infektion } \\
\text { unbekannt }\end{array}$ & 0 & 0 & Leukoderma & . \\
\hline 23 & $\begin{array}{l}\text { Gösta L. } \\
\mathbf{2 8} \% 1903\end{array}$ & $\begin{array}{c}\text { Infektion } \\
\text { unbekannt }\end{array}$ & 0 & 0 & Leukoderma & $\cdot$ \\
\hline 24 & $\begin{array}{l}\text { Adolf } \mathrm{H} \\
{ }^{28} / 5 \\
1904\end{array}$ & $\begin{array}{l}\text { Infektion } \\
\text { unbekann }{ }^{t}\end{array}$ & 0 & 0 & $\begin{array}{l}\text { Psoriasis palm., } \\
\text { Leukoderma }\end{array}$ & · \\
\hline 25 & $\underset{13 / 9}{\text { Marta S. }}$ & $\begin{array}{l}9 \text { Monate } \\
\text { ungefähr }\end{array}$ & 0 & $\begin{array}{c}\text { Hg-Säckchen } \\
\text { à } 8 \underset{\text { g. } 40+35}{\text { Tage }}\end{array}$ & $\begin{array}{l}\text { Pap. muc. vulv. } \\
\text { ani tonsill, Ery- } \\
\text { thema pap., Pso- } \\
\text { riasis plant., } \\
\text { Leukoderma }\end{array}$ & - \\
\hline
\end{tabular}


Klin. Beobachtungen über die Prognose der kong. Syphilis. 175

\begin{tabular}{|c|c|c|c|c|c|c|}
\hline \multicolumn{6}{|c|}{$\begin{array}{llll}K & i & n & d\end{array}$} & \multirow{3}{*}{ Bemerkungen } \\
\hline \multicolumn{2}{|c|}{ Bei der Geburt } & \multicolumn{4}{|c|}{ Verla $\mathrm{af}$} & \\
\hline Symptorne & $\begin{array}{l}\text { Sero- } \\
\text { reaktion }\end{array}$ & I. Jahr & If. Jahr & III. Jahr & IV. Jahr & \\
\hline . & - & - & - & - & - & - \\
\hline $\begin{array}{c}\text { S-frei } \\
4: 340 \mathrm{~g}\end{array}$ & - & $\begin{array}{l}{ }^{0} \\
\text { Otitis bilat. } \\
\text { Morbilli }\end{array}$ & $\stackrel{0}{\text { Diphterie }}$ & 0 & 0 & - \\
\hline $\begin{array}{l}\text { Exanth. } \\
\text { pap., } \\
\text { Koryza }\end{array}$ & - & $\begin{array}{c}0 \\
\text { Morbilli }\end{array}$ & $\mid \begin{array}{lll}0 & 11 / 8 & 1903 \text { in } \\
\dagger & \\
\text { Pneumonie }\end{array}$ & - & - & $\begin{array}{l}\text { Bei der Sektion keine } \\
\text { Zeichen von Syphilis. }\end{array}$ \\
\hline $\begin{array}{l}\text { Nicht aus- } \\
\text { getragen, } \\
\text { S-frei } \\
\mathbb{Z} \cdot 100 \mathrm{~g}\end{array}$ & - & $\begin{array}{c}0 \\
\text { Diphtherie }\end{array}$ & 0 & 0 & 0 & $\begin{array}{l}\text { 10/1 1910. S-frei. W. R. - } \\
\text { Juni 1912. S-frei. Gut } \\
\text { entwickelt. }\end{array}$ \\
\hline $\begin{array}{l}\text { Exanth. pap. } \\
\text { univers. +, } \\
\text { Pap.muc. ad } \\
\text { anum +, } \\
\text { Koryza' }\end{array}$ & $\cdot$ & $\begin{array}{c}0 \\
\text { Diphtherie }\end{array}$ & $\begin{array}{l}0 \\
+ \\
+\end{array}$ & - & - & $\begin{array}{l}\text { Bei der Sektion keine } \\
\text { Zeichen von Syphilis. }\end{array}$ \\
\hline $\begin{array}{c}\text { S-frei } \\
\text { 2. } 350 \text { g. Nach } \\
3 \text { M. Exanth. } \\
\text { pap. + +. } \\
\text { Koryza }\end{array}$ & . & $\begin{array}{c}0_{19} / 111904 \text { in } \\
\text { Diphtherie }\end{array}$ & - & - & • & - \\
\hline $\begin{array}{l}\text { Pap. muc. } \\
\text { vulv. et } \\
\text { ani,Koryz. }\end{array}$ & - & $\begin{array}{c}0 \\
\text { Diphtherie }\end{array}$ & 0 & 0 & 0 & $\begin{array}{l}\text { 10/1 1910. S-frei. W. R. - } \\
\text { Juni 1912. S-frei. Gut } \\
\text { entwickelt. }\end{array}$ \\
\hline $\begin{array}{l}\text { Exanti.pap. } \\
\text { univ., Pap. } \\
\text { muc. lab. } \\
\text { sup.,Eoryz }\end{array}$ & - & $\begin{array}{c}0 \\
\text { Diphtherie }\end{array}$ & 0 & 0 & 0 & $\begin{array}{l}\text { Juni 191\%. S-frei. } \\
\text { Gut entwickelt. }\end{array}$ \\
\hline $\begin{array}{c}\text { Exanth.pap. } \\
\text { univers., } \\
\text { Koryza } \\
\text { Koryza, }\end{array}$ & - & $\begin{array}{c}0 \\
\text { Diphtherie }\end{array}$ & 0 & $\mathbf{0}$ & 0 & $\begin{array}{c}\text { Juni 1912. Die mittleren } \\
\text { Schneidezähne des Ober- } \\
\text { kiefers etw, unregelmäßig; } \\
\text { ubrigens S-frei. }\end{array}$ \\
\hline $\begin{array}{l}\text { Pap. muc. } \\
\text { ani }\end{array}$ & • & $\begin{array}{c}0 \\
\text { Diphtherie }\end{array}$ & 0 & 0 & 0 & $\begin{array}{c}\text { Juni 1912. S-frei. Gut } \\
\text { entwickelt. }\end{array}$ \\
\hline $\begin{array}{l}\text { Psoriasis } \\
\text { palm. et } \\
\text { plant., } \\
4 \cdot 300 \mathrm{~g}\end{array}$ & • & $\begin{array}{c}0 \\
\text { Diphtherie }\end{array}$ & 0 & 0 & $\begin{array}{l}0 \\
\text { Chronische } \\
\text { Bronchitis }\end{array}$ & $\begin{array}{l}16 / 91910 . \text { S-frei. W. R. } \\
\text { † Dez. } 1910 \text { in Pneumonie. }\end{array}$ \\
\hline S-frei & $\cdot$ & 0 & 0 & 0 & 0 & $\begin{array}{l}\text { Juni 1912. S-frei. Gut } \\
\text { entwickelt. }\end{array}$ \\
\hline
\end{tabular}




\begin{tabular}{|c|c|c|c|c|c|c|}
\hline \multirow{3}{*}{ Nr. } & \multirow{3}{*}{$\begin{array}{c}\text { Na m e } \\
\text { und } \\
\text { Geburtstag }\end{array}$} & \multicolumn{5}{|c|}{$M u^{\prime} t \quad t r$} \\
\hline & & \multirow{2}{*}{$\underset{\text { Syphilis }}{\text { Alter der }}$} & \multicolumn{2}{|c|}{ Behandlung } & \multirow[b]{2}{*}{ Erscheinnngen } & \multirow[b]{2}{*}{ Seroreaktion } \\
\hline & & & $\begin{array}{c}\text { vor der } \\
\text { Gravidität }\end{array}$ & $\begin{array}{l}\text { während der } \\
\text { Gravidität }\end{array}$ & & \\
\hline 26 & $\begin{array}{l}\text { Nils M. } \\
17 / 11905\end{array}$ & 2 Jahre & $\begin{array}{c}\text { Hg- Säckchen } \\
\text { à } 8 g \\
40+40+40 \text { Tage }\end{array}$ & 0 & $\begin{array}{c}\text { Pap. muc. vulv. } \\
\text { Roseola, } \\
\text { Leukoderma }\end{array}$ & . \\
\hline 27 & $\underset{5 / 1}{\text { Ruth } O}$ & $\begin{array}{l}9 \text { Monate } \\
\text { ungefäbr }\end{array}$ & 0 & $\begin{array}{l}\text { Hg-Säckchen } \\
\text { à } 8 g \text { Tage }\end{array}$ & $\begin{array}{c}\text { Pap. muc. vulv. } \\
\text { adenit., } \\
\text { Leukoderma }\end{array}$ & • \\
\hline 28 & $\underset{16 \% 1905}{\operatorname{Dagmar} G}$ & $11 / 2$ Jahre & $3 \underset{\text { Schmier- }}{\text { kuren }}$ & 0 & $S$ frei & $\cdot$ \\
\hline 299 & $\begin{array}{l}\text { Greta R. } \\
15 / 81905\end{array}$ & $11 / 4$ Jahre & 1 Schmierkur & $\begin{array}{l}2 \text { Schmier- } \\
\text { kuren }\end{array}$ & Leukoderma & - \\
\hline 30 & $\begin{array}{l}\text { Josef } B . \\
20 / 71905\end{array}$ & $\begin{array}{l}\text { Infektion } \\
\text { unbekannt }\end{array}$ & 0 & 0 & $\begin{array}{c}\text { Roseola, } \\
\text { Pap. muc. vulv. } \\
\text { Leukoderma }\end{array}$ & $\cdot$ \\
\hline 31 & $\begin{array}{l}\text { Arnold H. } \\
26 / 51906\end{array}$ & $\begin{array}{l}4 \text { Monate } \\
\text { ungefähr }\end{array}$ & 0 & $\begin{array}{c}\text { Hg. Säckchen } \\
\dot{\mathbf{a}} 8 \mathrm{~g} 30+13 \\
\text { Tage }\end{array}$ & $\begin{array}{c}\text { Sklerose, } \\
\text { Papulae roseola }\end{array}$ & • \\
\hline 32 & $\begin{array}{l}\text { Seth L. } \\
17 / 91906\end{array}$ & 3 Jahre & $\begin{array}{l}6 \text { Schmier- } \\
\text { kuren }\end{array}$ & 0 & S-frei & • \\
\hline 33 & $\begin{array}{l}\text { Karin E. } \\
18 / 1 \\
1907\end{array}$ & 6 Jahre & $\begin{array}{l}2 \underset{\text { Schmier- }}{\text { kuren }} \\
\text { t }\end{array}$ & $\begin{array}{c}\text { Hg-Säckchen } \\
\text { à } 8 \text { g } 40+40 \\
\text { Tage }\end{array}$ & $\begin{array}{l}\text { Ulcera gum- } \\
\text { mosa, Papulo- } \\
\text { tubercula, } \\
\text { Leukoderma }\end{array}$ & - \\
\hline 34 & $\begin{array}{l}\text { Ella B. } \\
5 / 91906\end{array}$ & $18 / 4$ Jahre & $\begin{array}{c}\text { Hg-Säckehen } \\
\text { à } 8 \text { g } 45 \text { Tage }\end{array}$ & $\begin{array}{l}\text { Hg-Säckchen } \\
\text { à } 8 \text { g } 40 \text { Tage }\end{array}$ & $\begin{array}{l}\text { Roseola } \\
\text { annulata }\end{array}$ & $\cdot$ \\
\hline 35 & $\underset{1 / 41907}{\text { Harriet S. }}$ & 2 Jahre & $\begin{array}{c}\text { Hg. Säckchen } \\
\text { à } 8 \text { g 40 Tage } \\
+11 \text { Injektionen }\end{array}$ & 0 & S-frei & - \\
\hline
\end{tabular}


Klin. Beobachtungen über die Prognose der kong. Syphilis.

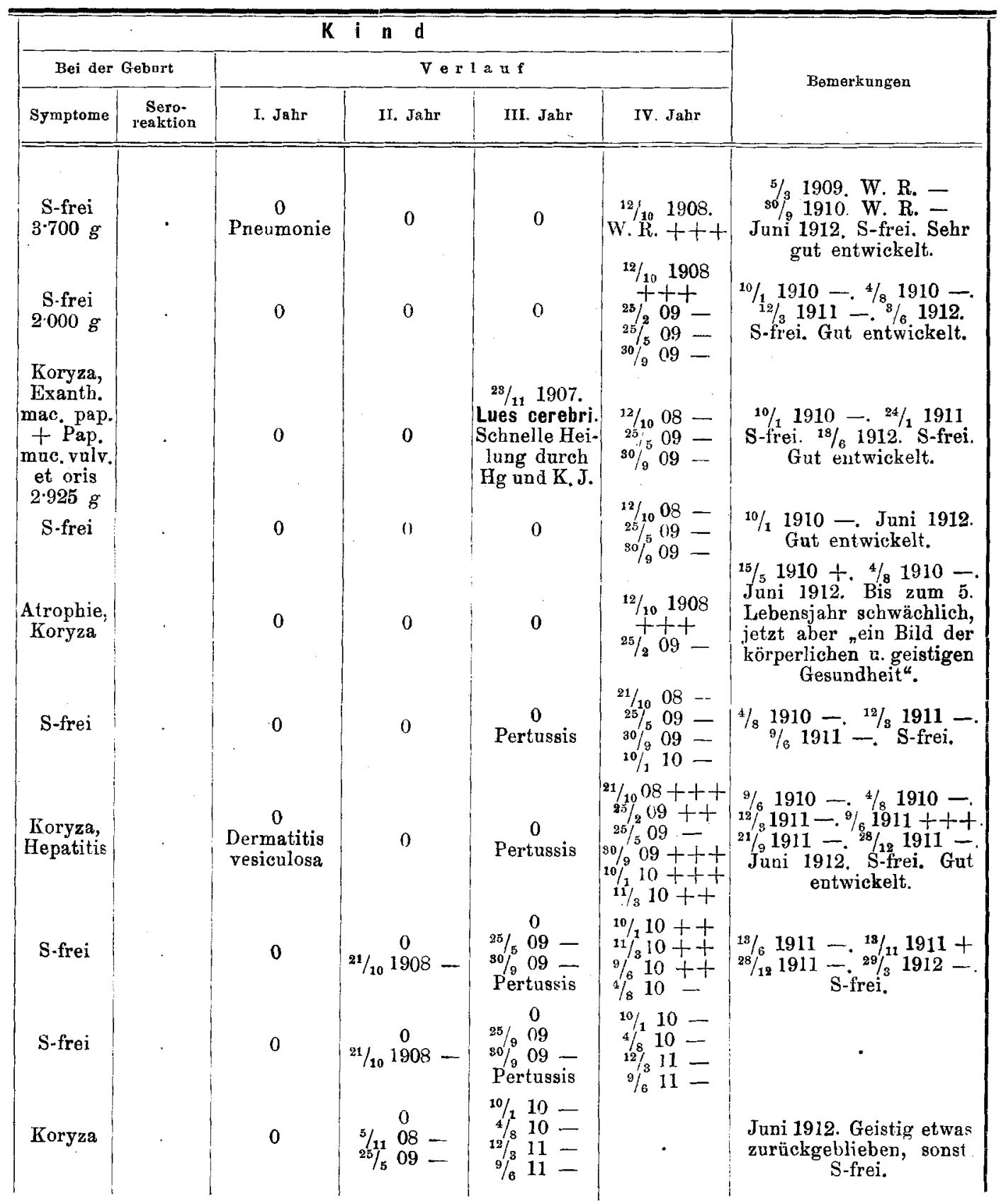

Arch. f. Darmat. n. Syph. Bd. CXVI. 


\begin{tabular}{|c|c|c|c|c|c|c|}
\hline \multirow{3}{*}{$\mathrm{Nr}}$. & \multirow{3}{*}{$\begin{array}{l}\text { Na me } \\
\text { und } \\
\text { Geburtstag }\end{array}$} & \multicolumn{5}{|c|}{$M u t t e r$} \\
\hline & & \multirow{2}{*}{$\begin{array}{l}\text { Aifer der } \\
\text { Syphilis }\end{array}$} & \multicolumn{2}{|c|}{ Beband lung } & \multirow[b]{2}{*}{ Erscheinungen } & \multirow[b]{2}{*}{ Seroreaktion } \\
\hline & & & $\begin{array}{l}\text { vor der } \\
\text { Gravidität }\end{array}$ & $\begin{array}{l}\text { während der } \\
\text { Gravidität }\end{array}$ & & \\
\hline 36 & $\begin{array}{l}\text { Bertil A. } \\
13 / 91907\end{array}$ & $\begin{array}{l}9 \text { Monate } \\
\text { ungefähr }\end{array}$ & 0 & $\begin{array}{l}2 \text { Injektionskur. } \\
+ \text { Hg-Säckchen } \\
\text { à } 8 \text { g } 40 \text { Tage }\end{array}$ & $\begin{array}{c}\text { Sklerose } \\
\text { oedema dur., } \\
\text { Adenit., Roseola }\end{array}$ & · \\
\hline 37 & $\begin{array}{l}\text { Erik A. } \\
22 / 2 \\
1907\end{array}$ & 3 Monate & 0 & 0 & $\begin{array}{l}\text { Sklerose, Lab. } \\
\text { sup. adenit., } \\
\text { Roseola }\end{array}$ & · \\
\hline 38 & $\underset{20 / 3}{\text { Margit } K}$ & $1 \mathrm{Jahr}$ & 0 & $\begin{array}{c}4 \text { Injektionen } \\
+ \text { Hg-Säckchen } \\
\dot{\text { a }} 8 \mathrm{~g} 40 \text { Tage }\end{array}$ & $\begin{array}{l}\text { Pap. muc. ani } \\
+ \text { Pap. syphilid. }\end{array}$ & $\cdot$ \\
\hline 39 & $\underset{30 \% 1}{\operatorname{Tyra}} 1908$ & $\begin{array}{l}\text { Infektion } \\
\text { unbekannt }\end{array}$ & 0 & 0 & Lues cerebri & $\cdot$ \\
\hline 40 & $\begin{array}{l}\text { Ella J } \\
2 / 81908\end{array}$ & 5 Monate & 0 & $\begin{array}{c}\text { Hg-Säckchen } \\
\text { à } 8 \text { g } 40 \text { Tage } \\
+4 \text { Sal. Hg }\end{array}$ & Sklerose & • \\
\hline 41 & $\begin{array}{l}\text { Gösta W. } \\
28 / 41908\end{array}$ & $\begin{array}{l}9 \text { Monate } \\
\text { ungefähr }\end{array}$ & $\theta$ & $\mid \begin{array}{c}40 \mathrm{Hg} \text {-Sä ckchen } \\
\grave{a} 8 \mathrm{~g}+4 \text { Inj. } \\
\text { Sal. Hg }\end{array}$ & $\begin{array}{c}\text { Sklerose, } \\
\text { Roseol, } \\
\text { Pap. muc. ani }\end{array}$ & $9 / 2108-$ \\
\hline 42 & $\begin{array}{l}\text { Nils } 0 \\
\mathbf{2 4} / 10 \\
1908\end{array}$ & $\begin{array}{l}\text { Infektion } \\
\text { unbekannt }\end{array}$ & 0 & 0 & $\begin{array}{c}\text { Erythema pap. } \\
\text { univ. + Pap. } \\
\text { muc. tonsill. }+ \\
\text { Leukoderma }\end{array}$ & $\begin{array}{l}9 / 1108+++ \\
15 \% / 109+++ \\
23 / 210 \underline{-}\end{array}$ \\
\hline 43 & $\begin{array}{l}\text { Gustav K. } \\
9 / 31909\end{array}$ & 2 Jahre & $\begin{array}{c}84 \text { Caps. } \\
\text { mergal. }+ \\
10 \mathrm{Sal.} \mathrm{Hg}+ \\
4 \mathrm{Mk} .\end{array}$ & $\begin{array}{c}\text { Hg-Säckchen } \\
\text { à } 8 \text { g } 28 \text { Tage }\end{array}$ & Leukoderma & - \\
\hline 44 & $\begin{array}{l}\text { Sven H. } \\
2 / 111909\end{array}$ & 9 Monate & 0 & 0 & $\begin{array}{l}\text { Roseola, } \\
\text { Pap. corp., } \\
\text { Eros. tons., } \\
\text { Leukoderma }\end{array}$ & $3 / 1210+t+$ \\
\hline 45 & $\underset{1 / 6}{\operatorname{Anna}} \underset{1909}{\operatorname{Lisa}} \mathrm{K}$. & 3 Jahre & 0 & 0 & $\begin{array}{l}\text { Leukoderma, } \\
\text { Papelreste in } \\
\text { der Vulva }\end{array}$ & $27 / 809-$ \\
\hline
\end{tabular}


Klin. Beobachtungen über die Prognose der kong. Syphilis. 179

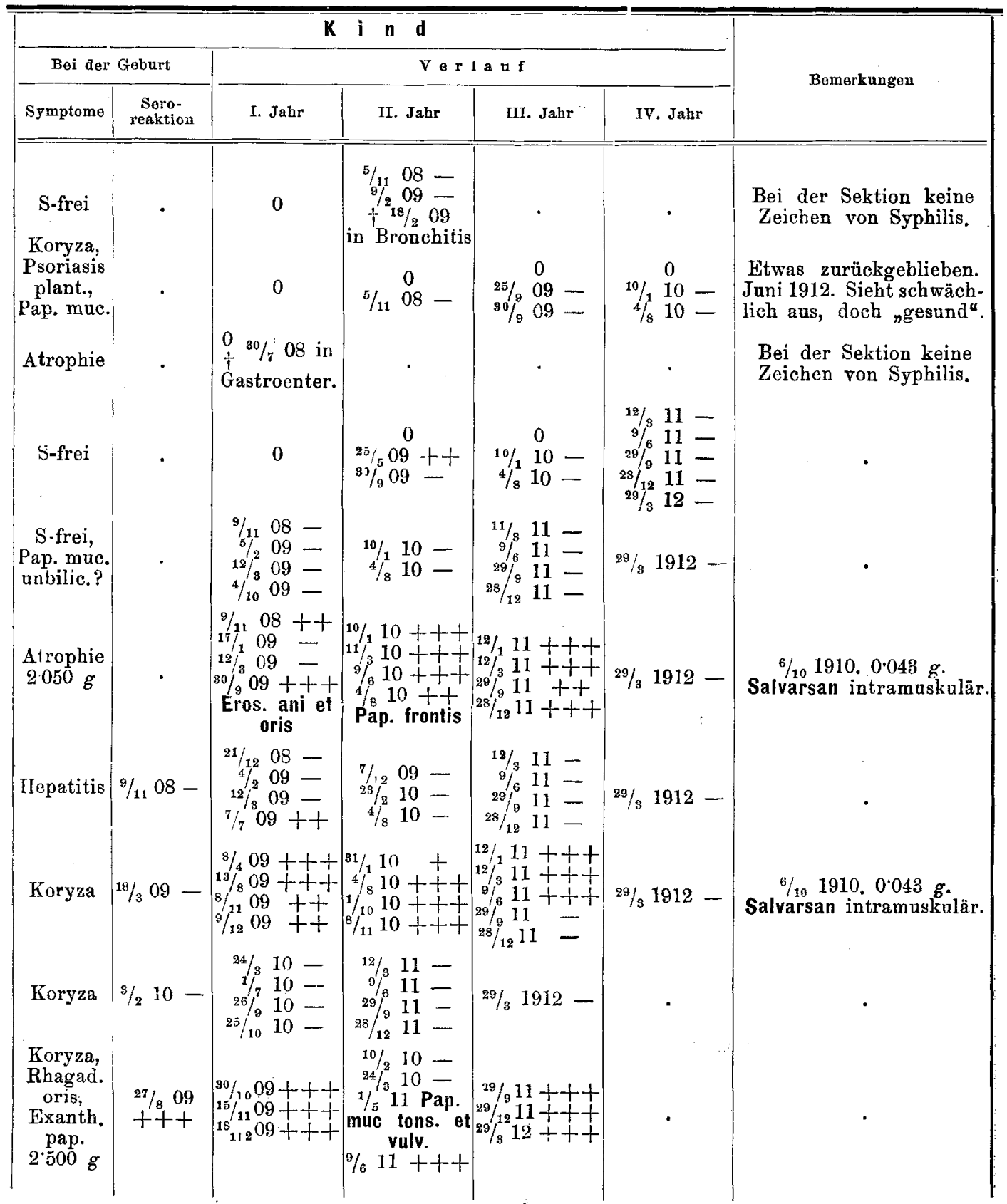




\begin{tabular}{|c|c|c|c|c|c|c|}
\hline \multirow{3}{*}{ Nr. } & \multirow{3}{*}{$\begin{array}{l}N \text { a m e } \\
\text { und } \\
\text { Geburtstag }\end{array}$} & \multicolumn{5}{|c|}{$M u t t e r$} \\
\hline & & \multirow[b]{2}{*}{$\begin{array}{l}\text { Alter der } \\
\text { Syphilis }\end{array}$} & \multicolumn{2}{|c|}{ Behand lung } & \multirow[b]{2}{*}{ Erscheinungen } & \multirow[b]{2}{*}{ Seroreaktion } \\
\hline & & & $\begin{array}{l}\text { vor der } \\
\text { Gravidität }\end{array}$ & $\begin{array}{l}\text { wäbrend der } \\
\text { Graviditatt }\end{array}$ & & \\
\hline 46 & $\begin{array}{l}\text { Josef A. } \\
2 \% / 51910\end{array}$ & 7 Monate & 0 & $10 \mathrm{Mk}$. & Leukoderma & $28 / 610+t+$ \\
\hline 47 & $\begin{array}{l}\text { Uno E. } \\
25 / 101909\end{array}$ & $1 / 2$ Jahre & 1 Injektionskur & $\begin{array}{c}\mathrm{Hg}-\mathrm{Säckchen} \\
\text { à } 8 \text { g } 40+40+40 \\
\text { Tage }\end{array}$ & Leukoderma & ${ }^{25} / 1009+++$ \\
\hline 48 & $\begin{array}{l}\text { Robert A. } \\
8 / 51910\end{array}$ & $\begin{array}{l}\text { Infektion } \\
\text { unbekannt }\end{array}$ & 0 & 0 & $\begin{array}{l}\text { Leukoderma, } \\
\text { Narben an der } \\
\text { Labb. maj. }\end{array}$ & $30 / 510+++$ \\
\hline 49 & $\begin{array}{l}\text { Vivi E. } \\
29 / 91909\end{array}$ & $\begin{array}{l}\text { Infektion } \\
\text { unbekannt }\end{array}$ & 0 & 0 & S-frei & $? / 09-$ \\
\hline 50 & $\begin{array}{l}\text { Sara P. } \\
12 / 1 \\
1910\end{array}$ & $\begin{array}{c}7 \text { Jahre } \\
\text { wahrscheinlich }\end{array}$ & 0 & 0 & S-frei & $13 / 410+++$ \\
\hline 51 & $\begin{array}{l}\text { Dagmar J. } \\
21 / 11 \quad 1910\end{array}$ & 2 Jahre & $7 \mathrm{Mk}$. & $\begin{array}{l}7 \mathrm{Mk} . \\
4 \mathrm{Mk} .\end{array}$ & $\begin{array}{c}\text { Papul. } \\
\text { aggregat. }\end{array}$ & $? / 10+++$ \\
\hline 52 & $\underset{2 \pi / 12}{\text { Erik F. }} 1910$ & $\begin{array}{l}\text { Infektion } \\
\text { unbekannt, } \\
\text { höchstens } \\
\text { I Jahr }\end{array}$ & 0 & 0.5 Salv. & $\begin{array}{c}\text { Roseola, } \\
\text { Pap. muc. vulv }\end{array}$ & - \\
\hline 53 & $\begin{array}{l}\text { Doris C. } \\
29 / 11 \\
1908\end{array}$ & & $\mathrm{U} \mathbf{n}$ & $\begin{array}{lllll}b & \mathbf{e} & \mathrm{k} & \mathrm{a} & \mathrm{n}\end{array}$ & $\mathrm{n}$ & \\
\hline 54 & $\begin{array}{l}\text { Helmer G. } \\
25 / 61907\end{array}$ & • & • & $\begin{array}{c}\text { Mutter und eine } \\
\text { Schwester } \\
\text { sollen W. R. } \\
+++ \text { haben }\end{array}$ & 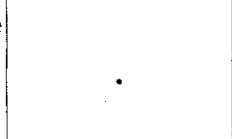 & . \\
\hline 55 & $\underset{14 / 6}{\text { Matts } H .}$ & $\begin{array}{l}\text { Infektion } \\
\text { unbekannt }\end{array}$ & 0 & 0 & S-frei & $28 / 1110+++$ \\
\hline 56 & $\underset{18 / 5}{\operatorname{Inga}} \mathrm{K}$. & 5 Monate & 0 & 0 & $\begin{array}{l}\text { Pap. muc. ani, } \\
\text { Exanth. pap. univ. } \\
+ \text { Adenit. ing. }+ \\
\text { Pap. capill. }+ \\
\text { Iritis dx. }\end{array}$ & $12 / 411+++$ \\
\hline
\end{tabular}


Klin. Beobachtungen über die Prognose der kong. Syphilis.

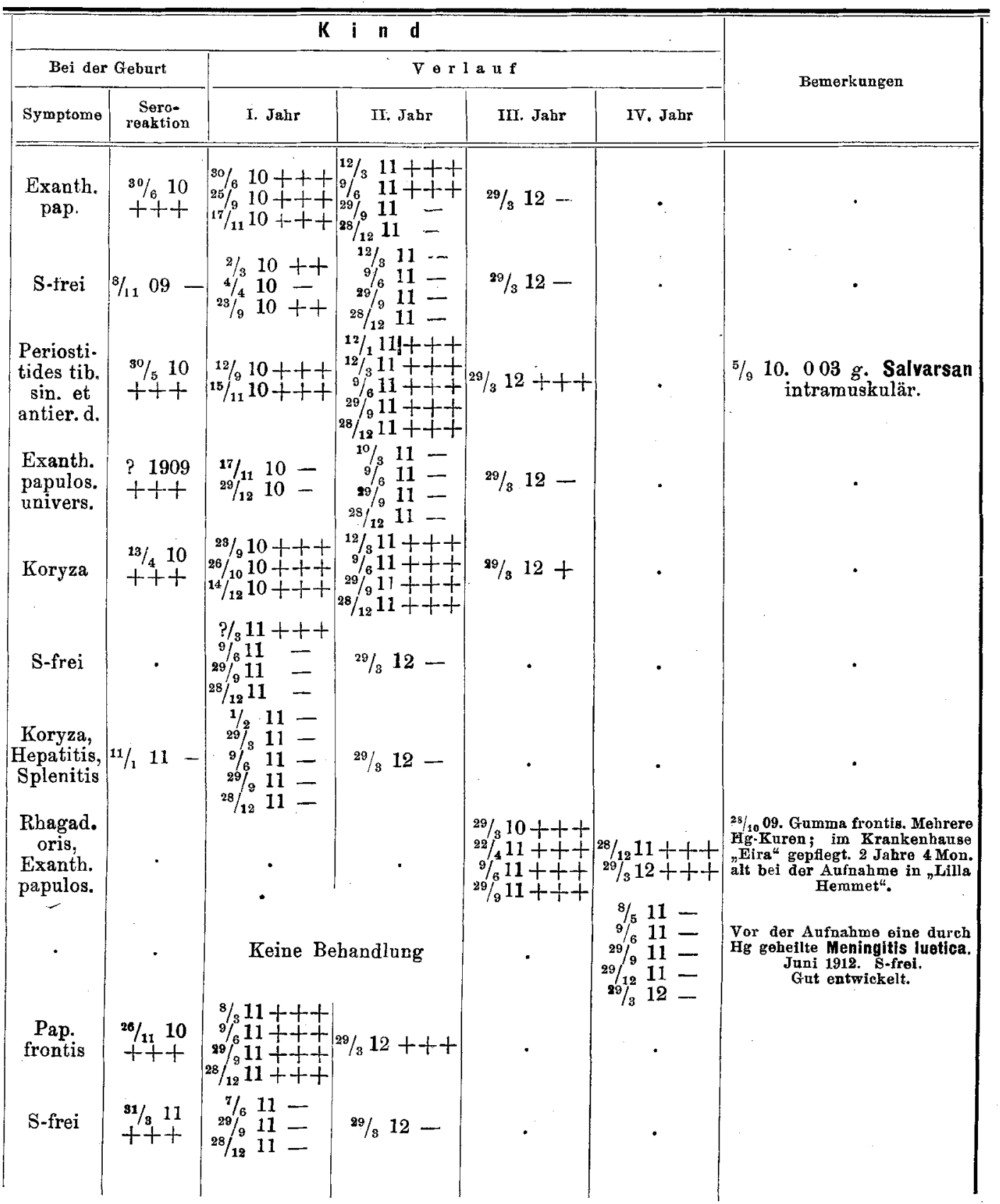


Mareus,

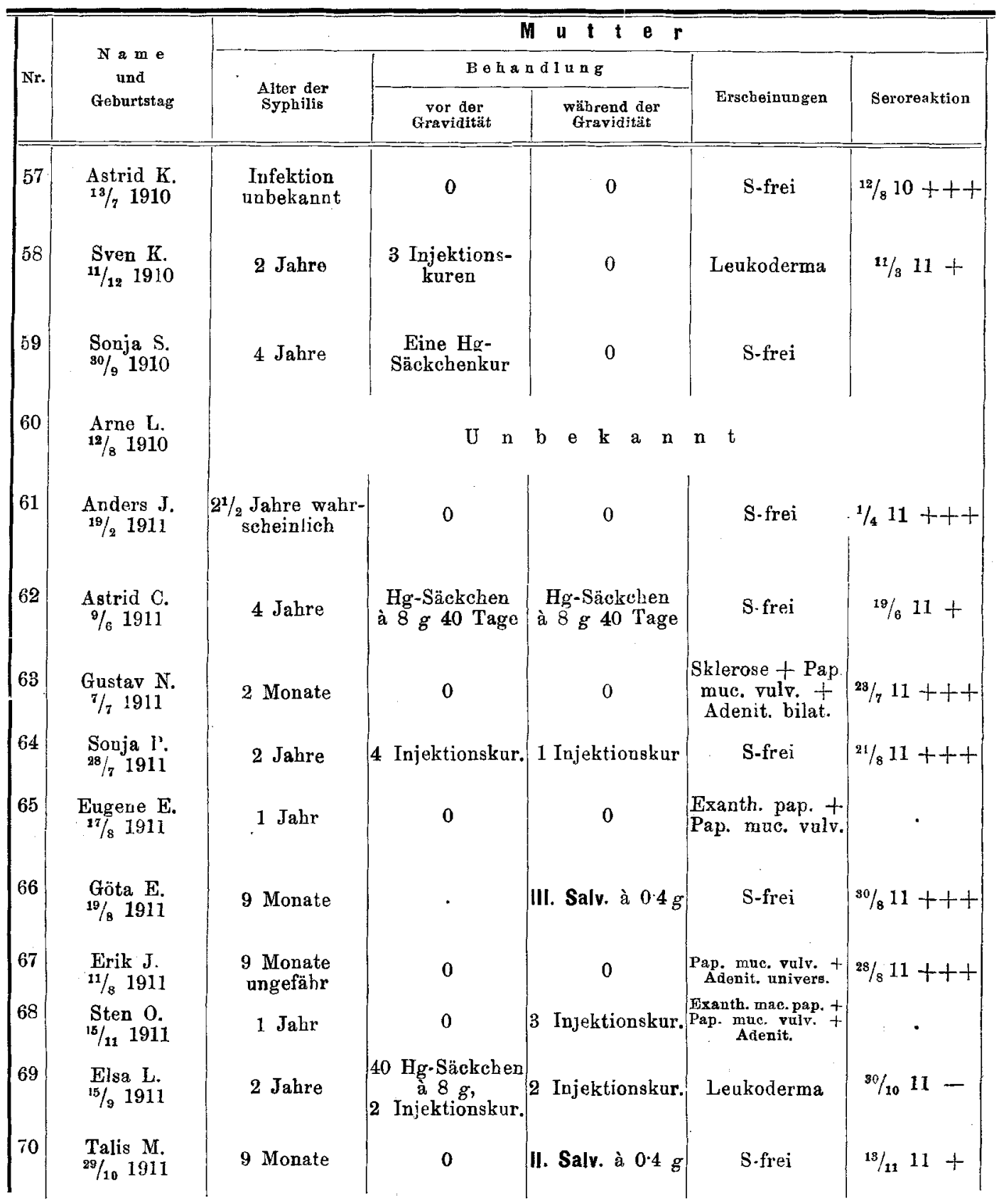


Klin. Beobachtungen über die Prognose der kong. Syphilis.

\begin{tabular}{|c|c|c|c|c|c|c|}
\hline \multicolumn{6}{|c|}{$\begin{array}{llll}K & i & \text { i }\end{array}$} & \multirow{3}{*}{ Bemerkungen } \\
\hline \multicolumn{2}{|c|}{ Bei der Geburt } & \multicolumn{4}{|c|}{$v e r l a u f$} & \\
\hline Symptome & $\begin{array}{l}\text { Sero- } \\
\text { reaktion }\end{array}$ & I. Jahr & II. Jahr & III. Jahr & IV. Jahr & \\
\hline $\begin{array}{l}\text { Korgza, } \\
\text { Exanth.pap. } \\
\text { Pap, mue. } \\
\text { ani,Splenitis }\end{array}$ & $\begin{array}{l}12 / 810 \\
++t\end{array}$ & • & $\begin{array}{l}7 / 711+++ \\
2 \% / 911++\end{array}$ & $\left\{\begin{array}{l}20 / 1211+++ \\
20 / 312+++\end{array}\right.$ & - & $\begin{array}{c}\text { Im Waisenhause } 23 / 8100.02 g \\
\text { and } 18 / 11100.035 g \text { Salvarsan. } \\
\text { Zwei Rezidive, hartnäekige } \\
\text { W. R. }+++\end{array}$ \\
\hline $\begin{array}{c}\text { Atrophie, } \\
\text { Koryza }\end{array}$ & $\begin{array}{l}11 / 311 \\
+++\end{array}$ & $\left|\begin{array}{l}13 / 611 \\
4 / 711-+ \\
29 / 911- \\
28 / 1211-\end{array}\right|$ & $29 / 312++$ & - & - & \\
\hline $\begin{array}{l}\text { Atrophie, } \\
\text { Koryza }\end{array}$ & $\begin{array}{l}10 / 1010 \\
+++\end{array}$ & $\begin{array}{l}8 / 1110+++ \\
23 / 1210+++ \\
24 / 211+++ \\
11 / 311-\end{array}$ & $\begin{array}{l}\dagger 1 / 811 \text { in } \\
\text { Kapillar- } \\
\text { bronchitis }\end{array}$ & - & - & $\begin{array}{l}\text { Bei der Sektion keine } \\
\text { Zeichen von Syphilis. }\end{array}$ \\
\hline $\begin{array}{l}\text { Exanth. } \\
\text { papulos. } \\
\text { univers. }\end{array}$ & . & - & $\begin{array}{l}19711+++ \\
2911+++ \\
28 / 911+++\end{array}$ & $29 / 312+$ & . & \\
\hline $\begin{array}{l}\text { Psor. piant. } \\
\text { et palm. + } \\
\text { pap. ani + } \\
\text { + Rhagad } \\
\text { oris } 2 \cdot 300 \mathrm{~g}\end{array}$ & $1 / 411+$ & $\begin{array}{c}9 / 711+++ \\
5 / 811+++ \\
29 / 911++ \\
28 / 911-\end{array}$ & $29 / 312-$ & . & - & \\
\hline S-frei & $20 / 611-$ & $\begin{array}{rrr}4 / 7 & 11 & - \\
9 / 9 & 11 & - \\
24 / 10 & 11 & + \\
28 / 12 & 11 & -\end{array}$ & $29 / 312++$ & - & - & $\begin{array}{l}\text { Keine Behandlung vor } \\
\text { dem fünften Monate. }\end{array}$ \\
\hline $\begin{array}{l}\text { Atrophie, } \\
\text { Hepatitis } \\
2.750 \mathrm{~g}\end{array}$ & $\begin{array}{l}24 / 711 \\
+++\end{array}$ & $\begin{array}{ll}15 / 9 & 11- \\
16 / 10 & 11- \\
28 / 12 & 11-\end{array}$ & $29 / 312++$ & . & - & \\
\hline S-frei & $\begin{array}{l}21 / 811 \\
+++\end{array}$ & $\begin{array}{ll}29 / 12 & 11- \\
29 / 8 & 12-\end{array}$ & - & - & - & \\
\hline $\begin{array}{l}\text { Koryza }+ \\
\text { Exanth. } \\
\text { papul. }\end{array}$ & . & $\left|\begin{array}{ll}30 / 10 & 11+++ \\
28 / 12 & 11+++ \\
20 / 3 & 12++-\end{array}\right|$ & - & - & - & \\
\hline $\begin{array}{c}\text { S-frei } \\
4: 300 \mathrm{~g}\end{array}$ & $80 / 8 \quad 11+$ & $\mid \begin{array}{ccc}29 / 9 & 11 & - \\
6 / 11 & 11 & - \\
28 / 12 & 11 & - \\
29 / 3 & 12 & +\end{array}$ & - & - & - & \\
\hline $\begin{array}{c}\text { S-frei } \\
3 * 900 \mathrm{~g}\end{array}$ & $28 / 811++$ & ${ }_{28}^{28} / 1011+t+$ & $29 / 3 \quad 12-$ & - & - & \\
\hline S-frei & $21 / 212-$ & - & . & - & - & \\
\hline $\begin{array}{c}\text { S-frei } \\
3 \cdot 200 \mathrm{~g}\end{array}$ & $30 / 1011-$ & $\left\{\begin{array}{lll}28 / 10 & 11 & - \\
21 / 1 & 12 & - \\
29 / 3 & 12 & -\end{array}\right.$ & - & - & - & \\
\hline $\begin{array}{l}\text { S-frei } \\
3 \cdot 220 \mathrm{~g}\end{array}$ & $13 / 1111-$ & $\begin{array}{ll}29 / 11 & 11- \\
21 / 1 & 12= \\
29 / 3 & 12-\end{array}$ & - & . & - & \\
\hline
\end{tabular}




\section{Literatur.}

Baisch. Die Vererbung der Syphilis auf Grund serologischer und bakteriologischer Untersuchungen. Münch. med. Woch. 1909. Nr. 38.

Bering, Fr. Über das Schicksal bereditär-syphilitischer Kinder (Lues hereditaria tarda?) Arch. f. Derm. u. Syph. Bd. CVI.

- Über kongenitale Syphilis, Entstehung, Erscheinungen und Behandlung. Sammlung zwangloser Abhandlungen aus dem Gebiete der Dermatol. und der Syphilidol. usw. Herausgegeben von Professor Dr. J. $\mathbf{J}$ a dass ohn in Bern. Bd. I. Heft 4.

Fournier, Alfred. Traité de la Syphilis. Paris 1898. Prophylaxie de la Syphilis. Paris 1903.

- En guérit-on? Paris 1906. Syphilis. Paris.

- À propos de la Prophylaxie et du traitement de l'Hérédo-

Fournier, Edmond. Stigmates dystrophiques de l'Hérédo-Syphilis. Paris 1898.

- Syphilis Héréditaire de l'age adulte. Paris 1912.

Hochsinger, Karl. Studien über die hereditäre Syphilis. Leipzig and Wien 1898.

- Die gesundheitlichen Lebensschicksale erbsyphilitischer Kinder. Wiener klin. Wochenschr. 1910. Nr. 24, 25.

Krefting, Rudolf. Sur l'hérédité de la syphilis. Arch. f. Derm. u. Syph. Bd. CX. Heft 3.

Lang, Ed. Lehrbuch der Geschlechtskrankheiten. Wiesbaden 1904.

Less er, Edmund. Lehrbuch der Haut- und Geschlechtskrankheiten. Leipzig 1896.

Marcus, Karl. Quecksilbertherapie und v. Wassermanns Reaktion. Arch. f. Derm. u. Syph. Bd. CVII. Heft 1-3.

Matzenauer, Rudolf. Die Vererbung der Syphilis. Arch. f. Derm. u. Syph. Ergänzungsheft. 1908 .

Oedmansson, Ernst. Till läran om syfilis congenita. Nord. med. ark. 1897.

Reuben, Mark S. Hereditäre Syphilis und Wassermannsche Reaktion. Arch. of Pediatr. Juni 1911. Ref. Derm. Woch. 1912, Nr. 7.

Rietschel. Über den Infektionsmodus bei der kongenitalen Syphilis. Med. Klinik. 1909. Nr. 18.

Rosenthal, 0 . Über Pflegeheime für hereditär-luetische Kinder. Arch. f. Derm. u. Syph. 1911.

S prinz, 0. Die Lebensaussichten der kongenital-luetischen Kinder. Derm. Wochenschr. 1912. Nr. 13, 14, 15.

Welander, E. Zur Frage: Wie kann man die durch eine syphilitische Schwangerschaft verursachte soziale Gefahr bekämpfen? Arch. f. Derm. u. Syph. Bd. LXIII. Heft 2, 3. holm $\overline{1907}$

- Ưber die Pflege kleiner hereditär-syphilitischer Kinder. Stock-

- Zur Frage der Behandlung der syphilitischen Krankheit. Beihefte zur Med. Klinik. 1909. Heft 6. 